\title{
Homological Representations of the Hecke Algebra *
}

\author{
R. J. Lawrence ${ }^{\star \star}$ \\ Department of Mathematics, Harvard University, Cambridge, MA 02138, USA \\ Received January 30, 1990; in revised form April 17, 1990
}

\begin{abstract}
In this paper a topological construction of representations of the $A_{n}^{(1)}$ series of Hecke algebras, associated with 2-row Young diagrams will be given. This construction gives the representations in terms of the monodromy representation obtained from a vector bundle on which there is a natural flat connection. The fibres of the vector bundle are homology spaces of configuration spaces of points in C, with a suitable twisted local coefficient system. It is also shown that there is a close correspondence between this construction and the work of Tsuchiya and Kanie, who constructed Hecke algebra representations from the monodromy of $n$-point functions in a conformal field theory on $\mathbf{P}^{1}$. This work has significance in relation to the one-variable Jones polynomial, which can be expressed in terms of characters of the Iwahori-Hecke algebras associated with 2-row Young diagrams; it gives rise to a topological description of the Jones polynomial, which will be discussed elsewhere [L2].
\end{abstract}

\section{Table of Contents}

1. Introduction . . . . . . . . . . . . . . . . . . . . 142

2. Topological Structure . . . . . . . . . . . . . . . . . . . . . . . 143

3. Translation into Algebra . . . . . . . . . . . . . . . . . . . . . . 148

3.1 Construction of Chain Complex

3.2 Form of the Boundaries . . . . . . . . . . . . . . . . . . . . . . 152

3.3 Action of the Braid Group . . . . . . . . . . . . . . . . . . . . . 155

3.4 Action of the Symmetric Group . . . . . . . . . . . . . . . . . . . 159

4. Examples . . . . . . . . . . . . . . . . . . . . . . . . . . . 160

$4.1 \mathrm{m=1}$ and the Alexander Polynomial. . . . . . . . . . . . . . . . . . 161

$4.2 m=2$ and Symmetrisation. . . . . . . . . . . . . . . . . . . . . . . 163

4.3 Symmetric Group Representations at $q=1$. . . . . . . . . . . . . . . 171

\footnotetext{
* This work was supported by a SERC studentship grant

$\star \star$ The author is a Lindemann Fellow of the English Speaking Union
} 
5. The General Case . . . . . . . . . . . . . . . . . . . . . . . . 173

5.1 Construction of a Subspace of Cohomology . . . . . . . . . . . . . . . 173

5.2 Braid Group and Symmetric Group Actions . . . . . . . . . . . . . . . 175

5.3 Selection of Subspace Using Limiting Behaviour . . . . . . . . . . . . . . 180

5.4 Relation with Work of Tsuchiya and Kanie . . . . . . . . . . . . . . . 183

6. Further Remarks. . . . . . . . . . . . . . . . . . . . . . . . . 189

\section{Introduction}

This paper is derived from [L1], which initiated the study of representations of Hecke algebras using geometric means. We restrict our attention here to only the most elementary application of the ideas presented. Representations of the Iwahori-Hecke algebras $H_{n}(q)$ are obtained by producing representations of the braid group $B_{n}$, and then showing that they factor through the Hecke algebra.

The braid group, $B_{n}$, is approached algebraically using the standard generators and relations; namely $\sigma_{1}, \ldots, \sigma_{n-1}$ with relations:

$$
\left.\begin{array}{ll}
\sigma_{i} \sigma_{i+1} \sigma_{i}=\sigma_{i+1} \sigma_{i} \sigma_{i+1} & \text { for } i=1,2, \ldots, n-2 \\
\sigma_{i} \sigma_{j}=\sigma_{j} \sigma_{i} & \text { for }|i-j|>1 .
\end{array}\right\}
$$

The Hecke algebra $H_{n}(q)$ of type $A_{n-1}^{(1)}$ is given as an algebra by the generators $\sigma_{1}, \ldots, \sigma_{n-1}$ with relations as above, together with the extra relations:

$$
\left(\sigma_{i}-1\right)\left(\sigma_{i}+q\right)=0
$$

for all $i=1,2, \ldots, n-1$. Note that some authors prefer to define $H_{n}(q)$ to have a relation in which the generators $\sigma_{i}$ all satisfy a quadratic relation with roots $-1, q$, in addition to the braid group relations, rather than the above relations where the roots are 1 and $-q$. This does not affect the essentials of the representation theory of the algebra $H_{n}(q)$; it simply introduces some sign changes.

The geometric approach to $B_{n}$ is as the fundamental group of the configuration space, $\widetilde{X}_{n}$, of $n$ unordered points in the complex plane, C. A representation of $B_{n}$ may thus be obtained from any vector bundle $E$ over the base $\widetilde{X}_{n}$ which is equipped with a flat connection, as the monodromy representation. In [J], it is seen how link polynomials may be expressed in terms of characters of the Hecke algebra $H_{n}(q)$. In particular, the (one-variable) Jones polynomial can be expressed as a linear combination of generically irreducible representations of $H_{n}(q)$ associated with 2-row Young diagrams. The group algebra of $H_{n}(q)$ reduces to $\mathbf{C S}_{n}$ when $q=1$, and as Wenzl demonstrated explicitly in [We], all irreducible representations of $S_{n}$ (as specified by Young diagrams with $n$ boxes) can be deformed to give representations of $H_{n}(q)$ as $q$ moves away from 1 . When $q$ is not a root of unity, the resultant representations are irreducible, and when $q$ is a root of unity, they may be reducible.

The initial motivation for the work was to better understand the significance of the Jones polynomial for links [FYHLMO]. This led on to an attempt to understand those representations of $H_{n}(q)$ associated with Young diagrams $\Lambda_{m}$ with $n$ squares in two rows, the second row consisting of $m$ squares. Such representations have also arisen in the context of conformal field theory, see [TK], where they arose as the monodromy representation of $B_{n}$, of a class of $n$-point functions. These $n$-point functions were found to satisfy a system of differential 
equations, from which it was deduced that the monodromy representation obtained factored through $H_{n}(q)$. The approach of this paper is to construct a fibre bundle over:

$$
X_{n}=\left\{\left(w_{1}, \ldots, w_{n}\right) \in \mathbf{C}^{n} \mid w_{1}, \ldots, w_{n} \text { are distinct }\right\}
$$

whose fibre is the configuration space of $m$ points in a punctured plane. Then there exists a two-parameter local coefficient system on the fibre, and hence a twoparameter family of homology groups of the fibre over any point in $\widetilde{X}_{n}$. There is a natural flat connection on the vector bundle over $\tilde{X}_{n}$ whose fibre is given by such homology groups. This gives rise to a two-parameter family of representations of $B_{n}$. Dually, one also has a two-parameter family of representations of $B_{n}$ on the associated cohomology spaces. It is found that when the two parameters $\alpha, q$ satisfy $\alpha=q^{-2}$, the resulting representation on cohomology contains as a subrepresentation, the representation of $H_{n}(q)$ associated with the Young diagram $\Lambda_{m}$. It is also found that the sub-representation may be constructed on a subspace which may be deduced naturally from behaviour when $\alpha$ is varied around $q^{-2}$, and $q$ is fixed.

When the cohomology is considered in terms of holomorphic functions with certain twistings, the flatness of a section of the vector bundle can be expressed by a system of first order partial differential equations. The comparison with the methods of Tsuchiya and Kanie gives rise to the prediction of the existence of an isomorphism between the two systems.

This paper is a shortened version of [L1], containing all the main results.

\section{Topological Structure}

In this section all the basic spaces necessary to enable the main theorems to be stated, will be defined. Recall that in Sect. $1, X_{n}, \widetilde{X}_{n}$ were defined to be:

$$
\begin{gathered}
X_{n}=\left\{\left(w_{1}, \ldots, w_{n}\right) \mid\left\{w_{i}\right\} \text { distinct in } \mathbf{C}\right\} \quad \text { (ordered points), } \\
\tilde{X}_{n}=X_{n} / S_{n}=\left\{\left\{w_{1}, \ldots, w_{n}\right\} \mid\left\{w_{i}\right\} \text { distinct in } \mathbf{C}\right\} \text { (unordered points). }
\end{gathered}
$$

We shall now construct a fibre bundle over $X_{n}$, whose fibre has (complex) dimension $m$, where $m \in \mathbf{N}$ is arbitrary, but fixed. For any $\mathbf{w} \in X_{n}$, let:

$$
Y_{\mathrm{w}, m}=\left\{\left(z_{1}, \ldots, z_{m}\right) \in X_{m} \mid z_{i} \neq w_{j} \text { for all } i \in\{1,2, \ldots, m\} \text { and } j \in\{1,2, \ldots, n\}\right\} \text {. }
$$

Then $Y_{w, m}$ defines a subset of $X_{m}$. In fact, the projection map:

$$
\begin{gathered}
X_{m+n}+n \\
\downarrow \\
X_{n}
\end{gathered}
$$

given by taking the first $n$ points only, of a set of $m+n$ points in $\mathbf{C}$, representing a point in $X_{m+n}$, has fibre $Y_{w, m}$ over the point $w \in X_{n}$. There is an obvious action of $S_{m}$ on $Y_{\mathrm{w}, m}$ given by permuting $z_{1}, \ldots, z_{m}$; this action will be important later in this section. Over each $w \in X_{n}$, a branched covering $\widetilde{Y}_{w, m}$ of $Y_{w, m}$, or equivalently, a local coefficient system $\chi_{w, m}$, will be defined as a function of a finite number of complex parameters. Now, a local coefficient system on $Y_{w, m}$, modelled on $\mathbf{C}$, is specified by a map, $\pi_{1}\left(Y_{\mathrm{w}, m}\right) \rightarrow \mathrm{C}^{*}$. However, $\pi_{1}\left(X_{m}\right)$ is the pure braid group on $m$ strings, and $\pi_{1}\left(Y_{w, m}\right)$ is the generalisation of this to the complex plane with $n$ points removed 
(namely the points $\left.w_{1}, \ldots, w_{n}\right)$. An element of $\pi_{1}\left(Y_{\mathrm{w}, m}\right)$ is given by $[\gamma]$ where:

$$
\gamma:[0,1] \rightarrow \mathrm{Y}_{\mathbf{w}, \boldsymbol{m}}
$$

is a collection of $m$ curves in $\mathbf{C} \backslash\left\{w_{1}, \ldots, w_{n}\right\}$, describing the motions of $z_{1}, \ldots, z_{m}$. Using the usual braid notation, we obtain a picture of $\gamma$ like that in Fig. 2.1, as an element of $B_{n+m}$ with the last $n$ strands straight, corresponding to $w_{1}, \ldots, w_{n}$ being fixed.

Fig. 2.1

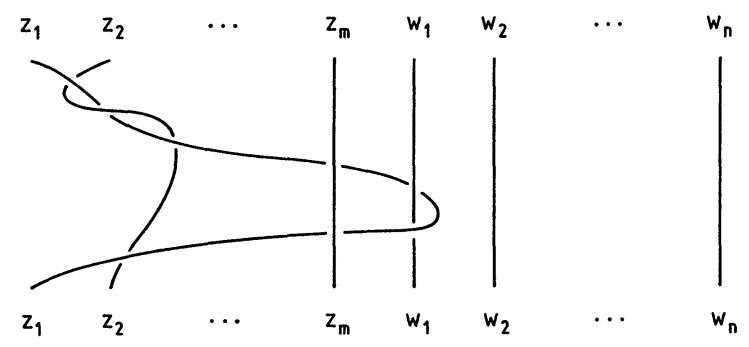

To make matters more precise at this stage, we will introduce some notation for particular elements of $\pi_{1}\left(Y_{w, m}\right)$. Consider only w's for which no two imaginary parts of $w_{i}$ 's coincide. Since $Y_{w, m}$ is unchanged when $w \in X_{n}$ is changed to $\sigma(w)$ for any $\sigma \in S_{n}$, it may be assumed, without loss of generality, that $w_{1}, \ldots, w_{n}$ are ordered so that their imaginary parts are increasing. Choose a base point in $Y_{w, m}$, say $\mathbf{z}^{0}$, such that:

$$
\mathfrak{I}\left(z_{1}^{0}\right)<\ldots<\mathfrak{I}\left(z_{m}^{0}\right)<\mathfrak{I}\left(w_{1}\right)<\ldots<\mathfrak{I}\left(w_{n}\right) .
$$

Let $\beta_{\lambda \mu}^{\prime}$ denote the element of $\pi_{1}\left(Y_{\mathrm{w}, m}\right)$ given by the curve fixing all $z_{i} \neq \lambda$, with $\lambda$ going round a curve in $\mathbf{C}$ which has winding number 1 about $\mu$, in a clockwise direction. Here, $\mu \in\left\{z_{j+1}, \ldots, z_{m}, w_{1}, \ldots, w_{n}\right\}$ and $\lambda=z_{j}$, where $1 \leqq j \leqq m$. The curve followed by $\lambda$, is defined by the statement that it does not cross any of the rays from points in $\left\{z_{1}, \ldots, \widehat{z_{j}}, \ldots, z_{n}, w_{1}, \ldots, w_{n}\right\} \backslash\{\mu\}$ in the direction $\mathbf{R}^{+}$. This defines $\beta_{\lambda, \mu}$ uniquely up to homotopy, as in Fig. 2.2.

Fig. 2.2

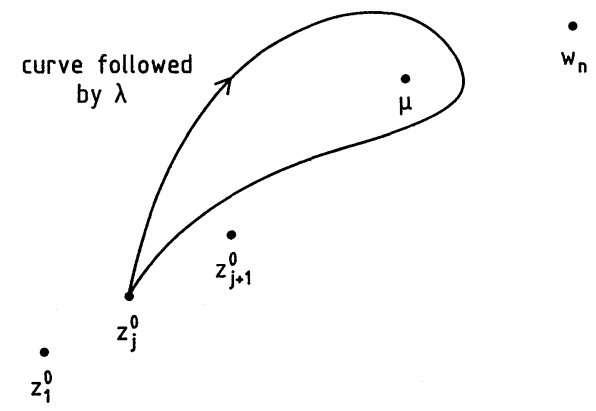

Any such element $[\gamma]$ of $\pi_{1}\left(Y_{\mathrm{w}, m}\right)$ defines an element of $B_{m+n}$ as mentioned above. It corresponds to a set of $m$ curves in $[0,1] \times\left(\mathbf{C} \backslash\left\{w_{1}, \ldots, w_{n}\right\}\right)$ given by $\left\{\left(t, \gamma_{i}(t)\right) \mid t \in[0,1]\right\}$ for $i=1,2, \ldots, m$. If we draw time, $t$, in a vertical direction, we obtain $m$ oriented curves in $\mathbf{R}^{3}$, connecting $m$ points on the plane $t=0$ to the corresponding set of $m$ points on the plane $t=1$. This picture may be viewed by projection onto the vertical plane $[0,1] \times \mathbf{i R}$, as indicated in Fig. 2.3. The twodimensional diagram so obtained gives, as illustrated in Fig. 2.4, the usual braid picture for $\beta_{\lambda \mu}$. In this picture time moves upwards, giving a natural orientation to the curves. 
Fig. 2.3
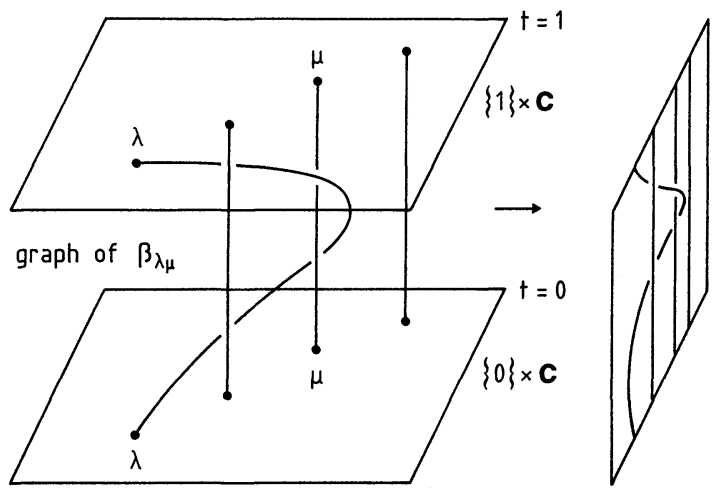

$[0,1] \times \mathbf{i R}$

Fig. 2.4

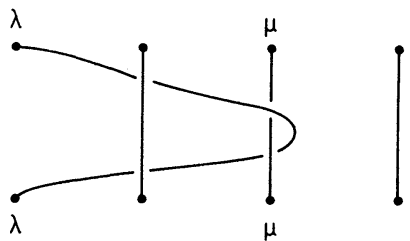

The generalised pure braid group $\pi_{1}\left(Y_{w, m}\right)$ is generated by $\left\{\beta_{\lambda \mu} \mid \lambda=z_{j}\right.$, $j \in\{1,2, \ldots, m\}$ and $\left.\mu \in\left\{z_{j+1}, \ldots, z_{m}, w_{1}, \ldots, w_{n}\right\}\right\}$. These generators satisfy generalised braid group relations, the details of which we shall not go into here. A onedimensional representation of $\pi_{1}\left(Y_{\mathrm{w}, m}\right)$ is given by specifying the images of these generators:

$$
\begin{gathered}
\pi_{1}\left(Y_{w, m}\right) \rightarrow \mathbf{C}^{*}, \\
\beta_{\lambda \mu} \mapsto q_{\lambda \mu}^{-1},
\end{gathered}
$$

where $q_{\lambda \mu}$ are non-zero complex numbers. There are no relations imposed on $q_{\lambda \mu}$, as can be seen by noting that:

$$
\prod_{i=1}^{m}\left(\prod_{\substack{j=1 \\ j>i}}^{m}\left(z_{i}-z_{j}\right)^{k_{z_{i} z_{j}}^{1}} \cdot \prod_{l=1}^{n}\left(z_{i}-w_{l}\right)^{\bar{k}_{z_{i} w_{l}}^{1}}\right)
$$

defines an analytic function of $z_{1}, \ldots, z_{m}$ with branch points where $z_{i}=z_{j}$ or $z_{i}=w_{l}$. This function multiplies by $q_{\lambda \mu}^{-1}$ as $\lambda$ goes around $\mu$ along the curve $\beta_{\lambda \mu}$. Here $\left\{k_{\lambda \mu}\right\}$ is defined so that:

$$
q_{\lambda \mu}=\exp \left(2 \pi i / k_{\lambda \mu}\right) .
$$

For any given set of non-zero complex numbers $\left\{q_{\lambda \mu}\right\}$ we can therefore define a local coefficient system $\chi_{\mathrm{w}, m}(\mathbf{q})$ on $Y_{\mathrm{w}, m}$, or equivalently, a branched covering $\widetilde{Y}_{\mathrm{w}, m}$. We have thus shown the following result:

Lemma 2.1. Given any $\mathbf{q}=\left\{q_{\lambda \mu}\right\}$ with $q_{\lambda \mu} \in \mathbf{C} \backslash\{0\}$ for any $\lambda$, $\mu$ of the form $\lambda=z_{j}$, $1 \leqq j \leqq m$ and $\mu \in\left\{z_{j+1}, \ldots, z_{m}, w_{1}, \ldots, w_{n}\right\}$, there exists a well defined local coefficient system $\chi_{w, m}(\mathbf{q})$ which twists by $q_{\lambda \mu}^{-1}$ around the curve represented by $\left[\beta_{\lambda \mu}\right] \in \pi_{1}\left(Y_{\mathrm{w}, m}\right)$. 
Let $E_{m}(q)$ be the vector bundle over $X_{n}$, whose fibre over the point $\mathbf{w} \in X_{n}$ is the vector space:

$$
H_{m}\left(Y_{\mathbf{w}, m}, \chi_{w, m}(\mathbf{q})\right)=E_{\mathbf{w}, m}(\mathbf{q}) \text {. }
$$

Since homology is homotopy invariant, there is a natural flat connection on this vector bundle. The monodromy of this connection gives rise to a representation of $\pi_{1}\left(X_{n}\right)=P_{n}$ on the homology. If $[\gamma] \in \pi_{1}\left(\widetilde{X}_{n}\right)$, then $\gamma$ defines a curve $[0,1] \rightarrow X_{n}$ such that $\gamma(0), \gamma(1) \in X_{n}$ differ from each other by a permutation. The natural connection on $E_{m}(\mathbf{q})$ then gives a parallel transport along $\gamma$ from the fibre over $\gamma(0)$ to that over $\gamma(1)$. However $\gamma(0), \gamma(1)$ differ by a permutation, and thus for suitable q (see below), we have $E_{\gamma(0), m}(\mathbf{q}) \cong E_{\gamma(1), m}(\mathbf{q})$.

As mentioned earlier in this section, there is a natural action of $S_{m}$ on $Y_{\mathrm{w}, m}$ given by permuting $z_{1}, \ldots, z_{m}$. This carries over to an action on $\widetilde{Y}_{w, m}$ so long as the local coefficient system $\chi_{w, m}(q)$ is preserved by the action of $S_{m}$. In particular, this requires that $q$ must be such that,

$$
\left.\begin{array}{ll}
q_{z_{i} z_{j}} \text { is independent of } i, j \in\{1,2, \ldots, m\}, & \text { for } i<j ; \\
q_{z_{i} w_{j}} \text { is independent of } i \in\{1,2, \ldots, m\}, & \text { for } j=1,2, \ldots, n .
\end{array}\right\}
$$

Going back to the action of $B_{n}$, if $[\gamma] \in \pi_{1}\left(\tilde{\mathrm{X}}_{n}\right)$ with $\gamma(1)=\sigma\left(\mathbf{w}_{0}\right), \gamma(0)=\mathbf{w}_{0}$, then $Y_{\mathrm{w}, m}$ $=Y_{\sigma\left(\mathbf{w}_{0}\right), m}$. The local coefficient system $\chi_{\sigma\left(\mathbf{w}_{0}\right), m}(\mathbf{q})$ on $Y_{\sigma\left(\mathbf{w}_{0}\right), m}$ is equivalent to a local coefficient system $\chi_{\mathrm{w}_{0}, m}(\sigma(\mathbf{q}))$ on $Y_{\mathrm{w}_{0}, m}$, where $\sigma(\mathbf{q})$ is defined by:

$$
[\sigma(\mathbf{q})]_{\lambda \mu}=\left\{\begin{array}{lll}
q_{\lambda \sigma^{-1}(\mu)} & \text { for } \mu \in\left\{w_{1}, \ldots, w_{n}\right\} ; \\
q_{\lambda \mu} & \text { for } \mu \in\left\{z_{1}, \ldots, z_{m}\right\} \backslash \lambda .
\end{array}\right.
$$

Hence if $\mathbf{q}$ is such that $\sigma(\mathbf{q})=\mathbf{q}$, then there is a natural isomorphism between:

$$
H_{m}\left(Y_{w_{0}, m}, \chi_{w_{0}, m}(\mathbf{q})\right)=E_{\gamma(0), m}(\mathbf{q}) \text { and } H_{m}\left(Y_{\sigma\left(\mathbf{w}_{0}\right), m}, \chi_{\sigma\left(w_{0}\right), m}(\mathbf{q})\right)=E_{\gamma(1), m}(\mathbf{q}) \text {. }
$$

Together with the parallel transport, we obtain a map:

$$
E_{\gamma(0), m}(\mathbf{q}) \rightarrow E_{\gamma(1), m}(\mathbf{q}) \cong E_{\gamma(0), m}(\mathbf{q})
$$

which thus gives rise to an action on $E_{\gamma(0), m}(\mathbf{q})$. Hence we obtain an action of $B_{n}$ on the fibre $E_{\gamma(0), m}(\mathbf{q})$ so long as:

$$
\sigma(\mathbf{q})=\mathbf{q} \forall \sigma \in S_{n} .
$$

This requires that $q_{z_{i} w_{j}}$ is independent of $j \in\{1,2, \ldots, n\}$ for all $i \in\{1,2, \ldots, m\}$. Hence we have:

Lemma 2.2. The natural connection on $E_{m}(q)$ induces natural actions of $B_{n}$ and $S_{m}$ on the fibres of $E_{m}(\mathbf{q})$, whenever q satisfies conditions (2.2) and (2.1), respectively. Hence there is an action of $B_{n} \times S_{m}$ on the fibres of $E_{m}(\mathbf{q})$, whenever $\mathbf{q}$ is of the form:

$$
q_{z_{i} z_{j}}=\alpha, \quad q_{z_{i} w_{k}}=\mathbf{q},
$$

where $i, j \in\{1,2, \ldots, m\}, k \in\{1,2, \ldots, n\}$ and $q, \alpha \in \mathbf{C}^{*}$.

When q satisfies (2.2), the action of $B_{n}$ on the fibres of $E_{m}(\mathbf{q})$ may be expressed more simply as follows. Let $\widetilde{E}_{m}(\mathbf{q})$ be the vector bundle over $\widetilde{X}_{n}$ whose fibre over a point $[\mathbf{w}] \in \tilde{X}_{n}$ is the vector space $E_{\mathbf{w}, m}(\mathbf{q})$. This is well-defined, so long as we identify the vector spaces corresponding to $\sigma(w)$ and $w$ as outlined above. Then the 
natural connection on $E_{m}(\mathbf{q})$ induces a natural connection on $\widetilde{E}_{m}(\mathbf{q})$. The two vector bundles $E_{m}(\mathbf{q}), \widetilde{E}_{m}(\mathbf{q})$ have identical fibres, but their base spaces differ, being $X_{n}$ and $\tilde{X}_{n}$, respectively. The action of $B_{n}$ on the fibres of $E_{m}(\mathbf{q})$ is now more simply expressed as the monodromy action of $\pi_{1}\left(\widetilde{X}_{n}\right)=B_{n}$ on the fibres of $\widetilde{E}_{m}(\mathbf{q})$. Since the fibres of $E_{m}(\mathbf{q})$ and $\widetilde{E}_{m}(\mathbf{q})$ are identical, the action of $S_{m}$ on $E_{m}(\mathbf{q})$ naturally identifies with an action on $\widetilde{E}_{m}(\mathbf{q})$, so long as $\mathbf{q}$ also satisfies (2.1), that is, for $\mathbf{q}$ 's of the form (2.3).

We are now in a position to give precise versions of the main theorems. The local coefficient system $\chi_{w, m}(\mathbf{q})$ (defined in Lemma 2.1) in which $\mathbf{q}$ takes the special values given by:

$$
q_{z_{i} z_{j}}=q^{-2}, \quad q_{z_{i} w_{k}}=q
$$

will be denoted by $\chi_{\mathbf{w}, m}(q)$. Here $\mathbf{q}$ refers to all the coefficients $q_{\lambda \mu}$, whereas $q$ indicates the special value of $q$ given by (2.4). This special local coefficient system satisfies both the conditions in Lemma 2.2 (that is (2.1) and (2.2)). Thus, by Lemma 2.2, natural actions of $B_{n}$ and $S_{m}$ exist on the fibres of $E_{m}(\mathbf{q})$, and, equivalently on the fibres of $\widetilde{E}_{m}(\mathbf{q})$.

Theorem 2.3. The monodromy action of $B_{n}=\pi_{1}\left(\tilde{X}_{n}\right)$ on the $S_{m}$-invariant part of the vector bundle $\widetilde{E}_{m}(q)$ contains, as a quotient, the representation of $B_{n}$ obtained from $\pi_{\Lambda_{m}}$ of $H_{n}\left(q^{-1}\right)$. The remaining component of the monodromy representation has dimension of order $1 / n$ times that of $\pi_{\Lambda_{m}}$.

The proof of this theorem occupies the next section and Sects. 5.1, 5.2. In Sect. 5, the local coefficient system is restricted to that of the form (2.4). However, since it is no more complicated to do so, the results of Sect. 3 will be proved for arbitrary local coefficient systems $\chi_{w, m}(\mathbf{q})$ satisfying (2.1) and/or (2.2) as appropriate. As it stands, the extent to which $\pi_{\Lambda_{m}}$ can be naturally picked out of the larger monodromy representation on $E_{\mathbf{w}, m}(q)^{S_{m}}$, is not clear. However the monodromy representation consists almost entirely of $\pi_{\Lambda_{m}}$, and in Sect. 5.3, a construction will be given which enables the sub-representation to be isolated, at least in certain cases. For any local coefficient system $\chi_{\mathbf{w}, m}(\mathbf{q})$ for which $\mathbf{q}$ satisfies (2.2), there is an proved in general, but from Theorem 5.13, the proof would be complete if it was verified that the symmetric part of the derived representation contains nothing other than $\pi_{A_{m}}$, by, for example, a dimension count.

action of $B_{n}$ on $E_{\mathbf{w}, m}(\mathbf{q})$, giving a family of representations of $B_{n}$, which contains the special case in which $\mathbf{q}$ is given by (2.4). There is an action of $S_{m}$ only on the twodimensional sub-family obtained from those $q$ of the form (2.4). In Sect. 5.3, a quotient representation of the special braid group representation is constructed from the family of braid group representations with neighbouring q's. This quotient representation will be referred to as the derived representation of the family. As was mentioned above, there is no action of $S_{m}$ defined on a general member of the family; however, the derived representation exists at the special value of $\mathbf{q}$ given by (2.4), and at this value of $\mathbf{q}$, an action of $S_{m}$ exists.

Conjecture 2.4. The symmetric part of the derived representation of the family of monodromy representations of $B_{n}$ on the vector bundles $\widetilde{E}_{m}(\mathbf{q})$, for $\mathbf{q}$ satisfying (2.2), at the value of $\mathbf{q}$ given by (2.4), is $\pi_{\Lambda_{m}}$.

This Conjecture is proved in Sect. 4.2 in the special case of $m=2$. For general $m$, it is shown in Sect. 5.3 (see Theorem 5.13) that the derived representation referred to above contains the representation $\pi_{\Lambda_{m}}$ of $H_{n}\left(q^{-1}\right)$. Conjecture 2.4 has not been 
Another point of view on the construction $\pi_{A_{m}}$ is given in Sect. 5.4, where it is shown that the following result holds (see Corollary 5.18).

Theorem 2.5. The monodromy action defined above is equivalent to that obtained from a system of differential equations of the form:

$$
\frac{\partial \mathbf{f}}{\partial w_{i}}=\sum_{j \neq i} \frac{\mathbf{c}_{i j} \mathbf{f}}{\left(w_{i}-w_{j}\right)}
$$

for a vector valued function $\mathbf{f}$ on $X_{n}$, where $\mathbf{c}_{i j}$ are constant matrices.

In Sect. 5.4, a comparison is made with the constructions of Tsuchiya and Kanie [TK] and Kohno [Ko], which also give rise to Hecke algebra representations. One of the main themes of their methods is the reduction to the study of a system of differential equations whose form is that given in Theorem 2.5.

In the next section we confine our attention to the homology construction involved in Theorem 2.3. The action of $B_{n} \times S_{m}$ on homology is determined by obtaining the full action on a suitable chain complex. The obvious basis for chains, in terms of iterated loops, is used, and recurrence relations are obtained from which matrices for all the actions can, in principle, be computed. The homology space is also identified with the kernel of a certain map on the space of chains, the matrix for which can also be determined from the recursion relations. The rest of the proof of Theorem 2.3 is outlined in Sect. 5, all the main steps being carried out explicitly for the special case of $m=2$, in Sect. 4.2.

To prove Theorem 2.3, a concrete basis for a subspace of cohomology is constructed, in Sect. 5.1, and the actions of $B_{n}$ and $S_{m}$ on this subspace are computed in Sect. 5.2. It is found that it is easier to work in the dual system, in terms of cohomology, rather than using homology. The action of $B_{n}$ on cohomology is dual to that on homology, and Theorem 2.3 is equivalent on cohomology to stating that a sub-representation of the monodromy representation of $B_{n}$ on cohomology, factors through $H_{n}(q)$ (rather than $H_{n}\left(q^{-1}\right)$ as in Theorem 2.3). It turns out that, in terms of a concrete basis for the subrepresentation, the action of $B_{n}$ is given in a particularly simple form, and it is then easy to deduce Theorem 2.3 .

\section{Translation into Algebra}

\subsection{Construction of Chain Complex}

In this sub-section we will construct a concrete chain complex on $Y_{w, m}$ with local coefficient system $\chi_{w, m}(\mathbf{q})$. On $\left\{z_{1}, \ldots, z_{m}, w_{1}, \ldots, w_{n}\right\}$ define an ordering so that:

$$
z_{i}<z_{j} \quad \text { iff } i<j ; \quad w_{k}<w_{l} \quad \text { iff } k<l ; \quad z_{i}<w_{k},
$$

for $i, j \in\{1,2, \ldots, m\}, k, l \in\{1,2, \ldots, n\}$. Choose a base-point $\mathbf{z}^{0}$, as in Sect. 2 , at which $\mathfrak{I}(\lambda)<\mathfrak{I}(\mu)$ whenever $\lambda, \mu \in\left\{z_{1}, \ldots, z_{m}, w_{1}, \ldots, w_{n}\right\}$ and $\lambda<\mu$.

For $\lambda<\mu$, let $\alpha_{\lambda \mu}$ denote the motion in $Y_{\mathrm{w}, m}$ in which all $\nu \neq \lambda, \mu$ are fixed and $\lambda, \mu$ move so that they transpose while following curves which are such that they do not $\left.w_{1}, \ldots, w_{n}\right\} \backslash\{\lambda, \mu\}$. We suppose that $\lambda, \mu$ swap round by going around each other in a clockwise direction. We thus have the diagrams found in Fig. 3.1 for $\alpha_{\lambda \mu}$, as a motion in the complex plane, and in terms of the braid picture. 


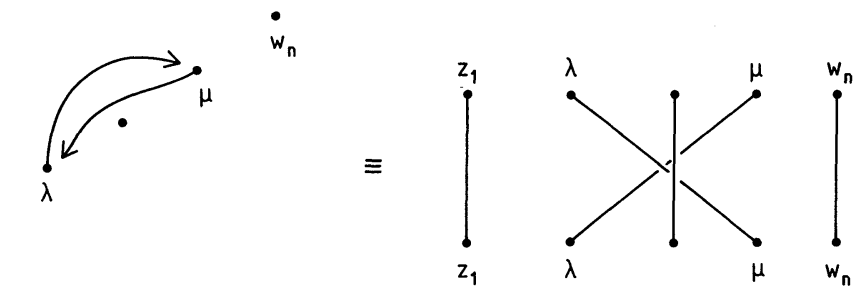

In Sect. 2 , the curves $\beta_{\lambda \mu}$ were similarly defined by motions in which $\lambda$ went once around $\mu$ in a clockwise direction. It is now obvious that $\beta_{\lambda \mu}=\alpha_{\lambda \mu}^{2}$.

Definition. For any $\mathbf{w} \in X_{n}$, and $r \in\{1,2, \ldots, m\}$, let:

$$
\begin{aligned}
& \mathscr{S}_{w}^{r}=\left\{\underline{\alpha}=\left(\alpha_{1}, \ldots, \alpha_{r}\right) \mid \alpha_{i} \in\left\{z_{i+1}, \ldots, z_{m}, w_{1}, \ldots, w_{n}\right\}\right\} ; \\
& \mathscr{T}_{w}^{r}=\left\{\underline{\alpha} \in \mathscr{S}_{w}^{r} \mid \alpha_{i} \in\left\{w_{1}, \ldots, w_{n}\right\} \text { and } \alpha_{i} \text { are all distinct }\right\} ; \\
& \mathscr{U}_{w}^{r}=\left\{\underline{\alpha} \in \mathscr{T}_{w}^{r} \mid \alpha_{1}>\alpha_{2}>\ldots>\alpha_{r}\right\} .
\end{aligned}
$$

This definition gives rise to sets $\mathscr{S}_{\mathrm{w}}^{r}, \mathscr{T}_{\mathrm{w}}^{r}, \mathscr{U}_{\mathrm{w}}^{r}$ of respective orders:

$$
\begin{gathered}
(n+m-1) \ldots(n+m-r), \\
n(n-1) \ldots(n-r+1), \\
n(n-1) \ldots(n-r+1) / r ! .
\end{gathered}
$$

For each $\underline{\alpha \in \mathscr{S}_{\mathrm{w}}^{r}}$ we will now proceed to define an embedding of the $r$-dimensional torus $T^{r}$ in $Y_{w, m}$. This torus will have $z_{s}=z_{s}^{0}$ whenever $s>r$. The map:

$$
\gamma_{\alpha}: T^{r} \rightarrow Y_{\mathrm{w}, m}, \quad\left(t_{1}, \ldots, t_{r}\right) \mapsto \gamma_{\underline{\alpha}}\left(t_{1}, \ldots, t_{r}\right)
$$

is then defined by giving the $i^{\text {th }}$ component of $\gamma_{\alpha}\left(t_{1}, \ldots, t_{r}\right)$ starting at $i=r$, and working back to $i=1$. This definition will be such that, for all $i$, this $i^{\text {th }}$ component is independent of $t_{1}, \ldots, t_{i-1}$. So we start by setting:

$$
\left(\gamma_{q}\left(t_{1}, \ldots, t_{r}\right)\right)_{r}=\beta_{z_{r} \alpha_{r}}\left(t_{r}\right) \text {. }
$$

For a particular value of $t_{r}$, we have defined the value of the position of $z_{r}$. The loop defined by $z_{i}$ as $t_{i}$ increases from 0 to 1 , with $t_{i+1}, \ldots, t_{r}$ fixed is defined so as to be a deformation of $\beta_{z_{i} \alpha_{i}}$. Suppose that $z_{i+1}, \ldots, z_{r}$ have already been defined as functions of $t_{i+1}, \ldots, t_{r}$. Then we deform $\beta_{z_{i} \alpha_{i}}$ continuously as $z_{i+1}, \ldots, z_{r}$ move from $z_{i+1}^{0}, \ldots, z_{r}^{0}$ due to the variation of $\left(t_{i+1}, \ldots, t_{r}\right)$ from $(0, \ldots, 0)$. The deformed curve is the curve we use to define the motion of $z_{i}$. Thus for $t_{1}, \ldots, t_{r}$ small, the values of $\left(\gamma_{\alpha}\left(t_{1}, \ldots, t_{r}\right)\right)_{i}$ are given by $\beta_{z_{i} \alpha_{i}}\left(t_{i}\right)$, for $1 \leqq i \leqq r$. When $t_{1}, \ldots, t_{r}$ are increased, we define $\gamma_{\alpha}$ so as to give a continuous embedding in $Y_{w, m}$.

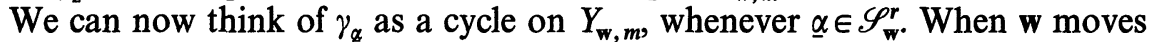
along a curve in $X_{n}$, the torus $\gamma_{\alpha}$ can be continuously deformed in a unique way (up to homotopy). This deformation corresponds at the level of homology to the natural connection discussed in Sect. 2. For each $\underline{\alpha \in \mathscr{S}_{\mathbf{w}}^{r}}$, it is now possible to lift the torus $\gamma_{q}$, which is embedded in $Y_{w, m}$, to $\widetilde{Y}_{w, m}$. When this is done, one obtains an embedding of $[0,1]^{r}$ in $\widetilde{Y}_{w, m}$ with base-point $\mathbf{z}^{0}$. Thus for any such $\underline{\alpha}, \gamma_{q}$ defines a chain on $\widetilde{Y}_{\mathrm{w}, m}$; elements of $\mathscr{S}_{\mathrm{w}}^{r}$ will often be loosely identified with chains.

The homology $H_{m}\left(Y_{w, m}, \chi_{w, m}(q)\right)$ may be computed in terms of the homology groups evaluated with a trivial local coefficient system, $\chi_{0}$, by using the following lemma. 
Lemma 3.1. The homology $H_{m}\left(Y_{\mathrm{w}, m}, \chi_{\mathrm{w}, m}(\mathbf{q})\right)$ can be computed from a finite dimensional chain complex $\mathscr{D}_{r}$ given by:

$$
\mathscr{D}_{r}=H_{r}\left(Y_{\mathrm{w}, m}, \chi_{0}\right)
$$

as the kernel of a suitably defined chain map $\delta: \mathscr{D}_{m} \rightarrow \mathscr{D}_{m-1}$.

Proof. Throughout this proof, $w \in X_{n}$ will be fixed, and we will abbreviate $Y_{w, m}$ to $Y$ and $\chi_{\mathrm{w}, m}(\mathbf{q})$ to $\chi_{\text {. }}$

It is also assumed that $\left\{\mathfrak{I}\left(w_{i}\right)\right\}$ are ordered as in Sect. 2. Consider the space:

$$
Y_{r}=\left\{\left(z_{r+1}, \ldots, z_{m}\right) \in X_{m-r} \mid z_{i} \neq w_{j} \text { for } r<i \leqq m \text { and } 1 \leqq j \leqq n\right\} .
$$

Then $Y_{0}=Y$ and for all $r=1,2, \ldots, m-1$, there is a fibration of $Y_{r-1}$ over $Y_{r}$, the fibres being one-dimensional. The fibre over $\left(z_{r+1}, \ldots, z_{m}\right) \in Y_{r}$ is the punctured plane $\mathbf{C} \backslash\left\{z_{r+1}, \ldots, z_{m}, w_{1}, \ldots, w_{n}\right\}$. A filtration of this fibre is defined by:

$$
\begin{aligned}
& K_{0}^{(r-1)}=\emptyset ; \\
& K_{1}^{(r-1)}=\bigcup_{\alpha>z_{r}}\left(\mathbf{R}^{+}+\alpha\right) ; \\
& K_{2}^{(r-1)}=\mathbf{C} \backslash\left\{z_{r+1}, \ldots, z_{m}, w_{1}, \ldots, w_{n}\right\},
\end{aligned}
$$

where $K_{1}^{(r-1)}$ is a union of $(n+m-r)$ cuts emanating from $z_{r+1}, \ldots, z_{m}, w_{1}, \ldots, w_{n}$ and $\mathbf{R}^{+}$denotes the positive real numbers. (For $r=m$, the above defines a filtration of $Y_{m-1}$.) This filtration defines a cell decomposition of the fibre, in which the $d$-dimensional cells are the components of $K_{d}^{(r-1)}-K_{d-1}^{(r-1)}$, so long as no two $z_{i}$ 's $(i<r)$ have identical imaginary parts. Whenever two or more $z_{i}$ 's have identical imaginary parts, we obtain non-distinct cuts, but it is still possible to define a filtration of the fibre by suitably deforming these cuts, in such a way that they no longer intersect.

Since there is a tower:

$$
Y=Y_{0} \rightarrow Y_{1} \rightarrow \ldots \rightarrow Y_{m-1} \rightarrow 0 .
$$

we can define a filtration, $\emptyset=K_{0} \leqq K_{1} \subseteq \ldots \leqq K_{2 m}=Y$ of $Y$, in which $K_{r}$ is of dimension $r$, and is obtained as the union of spaces of the form:

$$
K_{r_{0}}^{(0)} \times \ldots \times K_{r_{m-1}}^{(m-1)}
$$

over all $r_{0}, \ldots, r_{m-1} \in\{0,1,2\}$ with sum $r$. Here the product is identified in the natural way, with a subspace of $Y$, so that the $s^{\text {th }}$ term gives the possible values of $z_{s}$ in this subspace, once the values of $z_{s+1}, \ldots, z_{m}$ are fixed. Now $K_{i}^{(r)}-K_{i-1}^{(r)}$ is a disjoint union of Euclidean spaces for $i=1,2$ and any $r$, and thus so also is $K_{r}-K_{r-1}$. Hence $\left\{K_{r}\right\}$ may be viewed as providing a cellular decomposition of $Y$, in which the $r$-dimensional cells are given by the components of $K_{r}-K_{r-1}$. This is a slightly unconventional use of the term "cellular decomposition," since $Y$ is a non-compact space; however, $K_{r}-K_{r-1}$ is still a disjoint union of Euclidean spaces.

Since $K_{0}^{(i)}=\emptyset$ for all $i$, so $K_{r}=\emptyset$ whenever $r<m$. Also, $K_{m}$ consists of a disjoint union of products of one-dimensional rays. The components of $K_{m}$ are indexed by $\left(\alpha_{1}, \ldots, \alpha_{m}\right)$, where $\alpha_{i}$ indexes the components of $K_{1}^{(i-1)}$. That is the components of $K_{m}$ are indexed by $\underline{\alpha} \in \mathscr{S}_{\mathrm{w}}^{m}$. It is also seen that $K_{m+1}$ is a disjoint union of products of the form (3.1), with all but one $r_{i}$ being 1 , the remaining one being 2 . Hence $K_{m+1}$ naturally splits into $m$ parts, the $k^{\text {th }}$ part having components indexed by $\left(\alpha_{1}, \ldots, \alpha_{k}, \ldots, \alpha_{m}\right)$, where $\underline{\alpha \in \mathscr{S}_{\mathrm{w}}^{m}}$. This part is given by $r_{i}=1+\delta_{i k}$. 
The natural fibration of $Y_{r-1}$ over $Y_{r}$ has fibres homotopic to a wedge of $n+m-r$ circles. Hence if a tower:

$$
Z=Z_{0} \rightarrow Z_{1} \rightarrow \ldots \rightarrow Z_{m-1} \rightarrow 0,
$$

is defined so that the fibres in the fibration of $Z_{r-1}$ over $Z_{r}$ are wedges of $n+m-r$ circles, for all $r$, then the homology of $Y$ can be computed from that of $Z$. It is also easily seen that $K_{s+m}-K_{s+m-1}$ consists of a union of products of $K_{2}^{(r)}-K_{1}^{(r)}$ and $K_{1}^{(r)}$ spaces, with $s$ of the former type in each product. (Once again the product refers to the subspace of $Y$ with the $z$ 's as specified by the factors in each term.) Hence $K_{s+m}-K_{s+m-1}$ is homotopic to a wedge of $s$-torii. In particular, the components of $K_{s+m}-K_{s+m-1}$ are in 1-1 correspondence with those s-torii embedded in $Z$, obtained from one of the components of a slice of $Z$ by fixing one or more of the coordinates. The cohomology of $Y$ can be computed from the chain complex:

$$
\mathscr{D}^{r}=H_{c}^{r}\left(K_{r}-K_{r-1}\right),
$$

where $H_{c}^{*}$ denotes cohomology with compact support. In the case of a compact manifold this would follow from the standard theorem giving cohomology in terms of a cell decomposition. Although $Y$ is not compact, its cohomology can still be computed in this way, with the compactly supported cohomology $H_{c}^{*}$ replacing ordinary cohomology, since the interesting structure of $Y$ comes from those points $\mathbf{z}$ for which $z_{i}$ is near to $z_{i+1}, \ldots, z_{m}$ or $w_{1}, \ldots, w_{n}$, for each $i$. As noted above, $\mathscr{D}^{r}=0$ whenever $r<m$, while $\mathscr{D}^{m} \cong\left\langle\mathscr{S}_{\mathrm{w}}^{m}\right\rangle$ and $\mathscr{D}^{m+1}$ splits naturally as a product of $m$ spaces. In the dual picture, it is seen that the required homology is given by the homology of the chain complex $\mathscr{D}_{r} \cong \mathscr{D}^{2 m-r}$. Hence the $m$-dimensional homology is given by:

$$
\operatorname{ker}\left(\partial: \mathscr{D}_{m} \rightarrow \mathscr{D}_{m-1}\right) / \operatorname{Im}\left(\partial: \mathscr{D}_{m+1} \rightarrow \mathscr{D}_{m}\right) \cong \operatorname{ker}\left(\delta: \mathscr{D}^{m} \rightarrow \mathscr{D}^{m+1}\right) .
$$

Since $\mathscr{D}_{m-1}$ naturally splits into a product of $m$ vector spaces, this homology space is the intersection of the kernels of $m$ maps on $\mathscr{D}^{m} \cong\left\langle\mathscr{Y}_{w}^{m}\right\rangle$.

Finally, when the local coefficient system is trivial, all the boundary maps $\partial$ become trivial, and thus $\mathscr{D}_{r}$ may be identified with the space $H_{r}\left(Y_{w, m}, \chi_{0}\right)$ of the theorem.

In Sect. 3.2, the precise form of $\delta$ will be investigated. In particular, the components of $\delta$ corresponding to the decomposition of $\mathscr{D}^{m+1}$ noted above, will be evaluated. The above analysis in terms of cellular decompositions has a more geometrical formulation in which each cell in $K_{2 m-s}$ is represented by an embedding of a torus in $Y$, with base-point $\mathbf{z}^{0}$. Thus a cell in $K_{2 m-s}$ is given by a choice of $\alpha_{i}^{\prime}$ 's satisfying (3.2) for $i \in I$, where $I$ is some subset of $\{1,2, \ldots, m\}$ of order $s$. The subset $I$ labels those $i$ for which $r_{i}=1$ in (3.1), the rest of the $r_{i}$ 's being 2. Such a choice of $\alpha_{i}^{\prime}$ 's defines an embedding of $T^{s}$ in $Y$, as given by $\gamma_{\alpha}$. When this is lifted to $\widetilde{Y}$, it defines an embedding of $[0,1]^{s}$, and the different components of $\delta$, mentioned above, are obtained from the $s$ pairs of opposite faces in the boundary of such a hypercube. The tower given by the spaces $Y_{i}$ will play a central role in all the calculation of the rest of this section.

Let $\mathscr{C}_{r}$ denote the vector space generated by formal C-combinations of $\gamma_{\alpha}$ for $\underline{\alpha \in \mathscr{S}_{w}^{r}}$.

Then $\mathscr{C}_{r}$ can be identified with a subspace of the space of $r$-chains on $Y$.

As noted above $\mathscr{D}_{m} \cong \mathscr{C}_{m}$, and so $H_{m}\left(Y_{\mathrm{w}, m}, \chi_{\mathrm{w}, m}(\mathbf{q})\right)$ may be identified with a subspace of $\mathscr{C}_{m}$.

Before determining the actions of $B_{n}$ and $S_{m}$, it is necessary to first set up some notation. Whenever $\underline{\alpha} \in \mathscr{S}_{\mathrm{w}}^{r}$ and $s<r$, we denote by $\underline{\alpha}^{s}$, that element of $\mathscr{S}_{\mathrm{w}}^{s}$ given by 
truncating $\underline{\alpha}$, i.e. $\underline{\alpha}^{s}=\left(\alpha_{1}, \ldots, \alpha_{s}\right)$. If $\underline{\alpha} \in \mathscr{S}_{w}^{r}$ and $s>r$, then we shall denote the element $\left(\alpha_{1}, \ldots, \alpha_{s}\right) \in \mathscr{S}_{w}^{s}$ by $\underline{\alpha} \cdot \alpha_{r+1} \cdot \ldots \cdot \alpha_{s}$. For any $\underline{\alpha} \in \mathscr{S}_{w}^{r}$, the $r$-torus $\gamma_{\alpha}$ has $z_{r+1}, \ldots, z_{m}$ fixed, and a section on which $z_{r+1}$ is constant looks like $\gamma_{q}$, or a deformed version of it. In future sections we shall often write $\gamma_{\alpha} \cdot \alpha_{r+1}$ for $\gamma_{\alpha \cdot \alpha_{r+1}}$, where it is understood to mean that sections in which $z_{r+1}$ is constant are deformed versions of $\gamma_{\alpha}$ and also that $z_{r+1}$ varies around a loop $\beta_{z_{r+1} \alpha_{r+1}}$.

Under the motions of $z_{r+1}, \ldots, z_{m}, w_{1}, \ldots, w_{n}$ specified by $\alpha_{\lambda \mu}, \beta_{\lambda \mu}\left(z_{r+1} \leqq \lambda\right.$ $<\mu \leqq w_{n}$ ), the elements of $\mathscr{S}_{w}^{r}$ will transform to other chains, which are thus expressible as linear combinations of elements of $\mathscr{S}_{\mathrm{w}}^{\mathrm{r}}$. We denote by $A_{\lambda \mu}^{(r)}, b_{\lambda \mu}^{(r)}$ these transformations; they will be square matrices of order $\left|\mathscr{S}_{w}^{r}\right|$. The action of $S_{m}$ on the chains is specified in terms of the action of the generator which interchanges $z_{i}$ and $z_{i+1}$. This action is denoted by $j_{i+1}^{(r)}$ on the chains $\gamma_{\varepsilon}$ for $\underline{\alpha} \in \mathscr{S}_{w}^{r}$ with $r \geqq i+1$.

To obtain the homology, it is necessary to compute the boundary map $\delta: \mathscr{D}_{m}$ $\rightarrow \mathscr{D}_{m-1}$. This map is specified by $\left\{\pi_{i} \circ \delta: i=1,2, \ldots, m\right\}$, where $\pi_{i}$ is the projection of $\mathscr{D}_{m-1}$ onto that part in which $z_{i}$ is fixed. Then $\pi_{i} \circ \delta$ gives rise to a matrix with $\left|\mathscr{S}_{\mathbf{w}}\right|$ rows; these matrices are denoted by $\mathbf{D}_{i}^{(m)}$. The corresponding matrices, for $r=i, i+1, \ldots, m$, representing the boundary map on $\mathscr{C}_{r}$, with $z_{r+1}, \ldots, z_{m}$ fixed will be denoted by $\mathbf{D}_{i}^{(r)}$.

The above definition of the $A$ 's means that the action of $B_{n}$ on the chain space $\mathscr{C}_{m}$ is given by:

$$
\sigma_{i} \mapsto b_{w_{i} w_{i+1}}^{(m)} .
$$

Thus the aim of this section is to produce formulae from which $A_{w_{i} w_{i+1}}^{(m)}, b_{w_{i} w_{i+1}}^{(m)}$, $j_{i i+1}^{(m)}$ and $\mathbf{D}_{i}^{(m)}$ can be computed. We note that at zeroth order,

$$
b_{\lambda \mu}^{(0)}=q_{\lambda \mu}^{-1}, \quad A_{\lambda \mu}^{(0)}=1
$$

for any $\lambda, \mu$. Note also that $A_{\lambda \mu}^{(r)}$ is only well defined if the chains at w, and at the vector obtained from $w$ by transposing $\lambda$ and $\mu$, can be identified. That is, only if the local twists $\mathbf{q}$ are invariant under a transposition of $\lambda$ and $\mu$. In the future, $\left\{\gamma_{q} \mid \underline{\alpha} \in \mathscr{S}_{w}^{m}\right\}$ will often be referred to simply as $\mathscr{S}_{w}^{m}$.

\subsection{Form of the Boundaries}

The homology is the kernel of the boundary map, $\varepsilon$, on $\mathscr{C}_{m}$. When $\underline{\alpha} \in \mathscr{S}_{w}^{m}$, corresponding to a basis element of $\mathscr{C}_{m}$, the $i^{\text {th }}$ component of $\delta\left(\gamma_{\alpha}\right)$ is given by:

$$
\delta_{i}\left(\gamma_{\alpha^{i}} \cdot \alpha_{i} \alpha_{i+1} \cdot \ldots \cdot \alpha_{m}\right)=\delta_{i}\left(\gamma_{q^{i-1}} \cdot \alpha_{i}\right) \cdot \alpha_{i+1} \cdot \ldots \cdot \alpha_{m} .
$$

Here we know that $\delta_{i}\left(\gamma_{\alpha^{i}} \cdot \alpha_{i}\right)$ is the boundary of the torus $T^{i}$ in which a section with $z_{1}, \ldots, z_{i-1}$ fixed is given by the loop $\beta_{z_{i} \alpha_{i}}$ for $\alpha_{i}$. Its boundary is thus the difference of two $(i-1)$-torii corresponding to $\underline{\underline{i}}^{i-1}$ and its deformation when $z_{i}$ has gone around $\beta_{z_{i} a_{i}}$. Thus we obtain:

$$
\delta_{i}\left(\gamma_{\ell}\right)=\left(b_{z_{i} \alpha_{i}}^{(i-1)}-1\right) \gamma_{\alpha^{i-1}} \cdot \alpha_{i+1} \cdot \ldots \cdot \alpha_{m},
$$

where this denotes an $(i-1)$-cycle in which $z_{i}$ is constant at $z_{i}^{0}$. Hence a suitable matrix for $\delta$ is given by:

$$
\mathbf{D}_{i}^{(i)}=\left(b_{z_{i} z_{i+1}}^{(i-1)}-1|\ldots| b_{z_{i} z_{m}}^{(i-1)}-1\left|b_{z_{i} w_{1}}^{(i-1)}-1\right| \ldots \mid b_{z_{i} w_{n}}^{(i-1)}-1\right) .
$$

This is a partitioned matrix acting on $\left\langle\mathscr{S}_{w}^{i}\right\rangle$, and mapping it to $\left\langle\mathscr{S}_{w}^{i-1}\right\rangle$. The corresponding matrices $\mathbf{D}_{i}^{(r)}$ for $i \leqq r \leqq m$ acting on $\left\langle\mathscr{S}_{\mathbf{w}}^{r}\right\rangle$ are given by:

$$
\mathbf{D}_{i}^{(r)}=\left(\begin{array}{lll}
\mathbf{D}_{i}^{(r-1)} & & \\
& \ddots & \mathbf{D}_{i}^{(r-1)}
\end{array}\right) \text { for } i<r \leqq m .
$$


In all cases, the matrices are partitioned according to the values of $\alpha_{r} \in\left\{z_{r+1}, \ldots, z_{m}\right.$, $\left.w_{1}, \ldots, w_{n}\right\}$.

We have now shown how the action of the boundary map on the space of chains $\mathscr{C}_{m}$ can be computed; it is given by $\mathbf{D}_{i}^{(m)}$. In the rest of this work, $\mathbf{D}_{i}^{(m)}$ will be referred to simply as $\mathbf{D}_{i}$. It is given by a diagonal matrix with blocks $\mathbf{D}_{i}^{(i)}$ down the diagonal, where the separation into blocks is specified by the values of $\alpha_{i+1}, \ldots, \alpha_{m}$. Thus we obtain the lemma:

Lemma 3.2. The space $\bigcap_{i=1}^{m} \operatorname{ker}\left(\mathbf{D}_{i}\right) \subseteq \mathscr{C}_{m}$, where $\mathbf{D}_{i}$ are the matrices $\mathbf{D}_{i}^{(m)}$ specified by (3.3) and (3.4), is in 1-1 correspondence with the homology of $Y_{w, m}$ with the twisted local coefficient system $\chi_{\boldsymbol{w}, m}(\mathbf{q})$.

The reason for the non-trivial boundary map is that the local coefficient system is non-trivial. Thus when $\gamma_{\alpha}$ is lifted to $\widetilde{Y}_{\mathrm{w}, m}$ it gives rise to an embedded $m$-cube $[0,1]^{m} \rightarrow \widetilde{Y}_{w, m}$, and there are components in the boundary arising from each pair of opposite faces; that is from each $i \in\{1,2, \ldots, m\}$. See Fig. 3.2.

Fig. 3.2

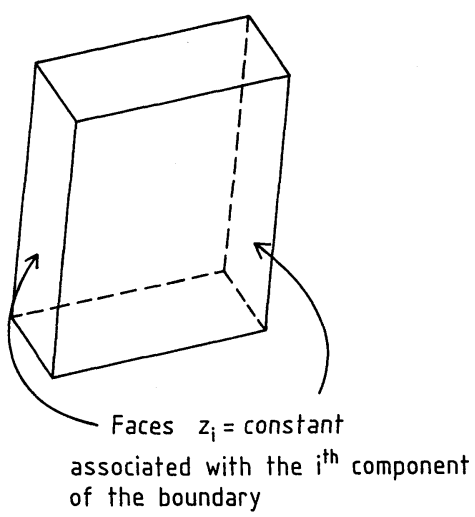

From the definitions of $A^{(r)}, b^{(r)}$ matrices, it is possible to derive the relations given in the lemma below. So as to avoid unnecessary indices, $A_{\lambda \mu}^{(r)}$ and $b_{\lambda \mu}^{(r)}$ have been abbreviated to $A_{\lambda \mu}$ and $b_{\lambda \mu}$, where it is always understood that they are transformations on $\left\langle\mathscr{S}_{\mathbf{w}}^{r}\right\rangle$ for a common value of $r$.

Lemma 3.3. The following relations hold between $A^{(r)}$ and $b^{(r)}$ matrices, for all $0 \leqq r \leqq m$ and $i, j, k, \lambda \in\left\{z_{r+1}, \ldots, z_{m}, w_{1}, \ldots, w_{n}\right\}$ :

(i) $A_{j k} b_{i j}=b_{i k} A_{j k}$;

(ii) $b_{i j} b_{i k} A_{j k}=A_{j k} b_{i j} b_{i k}$

(iii) $A_{i j} A_{j k} A_{i j}=A_{j k} A_{i j} A_{j k}$;

(iv) $A_{i j}^{2}=q_{i j} b_{i j}$;

(v) $A_{j k}$ commutes with $b_{i \lambda}$ whenever $\lambda<j$ or $\lambda>k$;

(vi) $b_{j k}^{-1} A_{j k} b_{i j}$ commutes with $b_{i \lambda}$ whenever $j<\lambda<k$;

(vii) $b_{j k}$ commutes with $b_{i \lambda}$ whenever $\lambda<j$ or $\lambda>k$;

(viii) $b_{i j}^{-1} b_{j k} b_{i j}$ commutes with $b_{i \lambda}$ whenever $j<\lambda<k$;

(ix) $b_{j k} b_{i j} b_{i k}=b_{i j} b_{i k} b_{j k}=b_{i k} b_{j k} b_{i j}$. 
Proof. (iv) The matrix $A_{i j}$ represents the action on $\left\langle\mathscr{Y}_{w}^{r}\right\rangle$ given by $\alpha_{i j} \in \pi_{1}\left(Y_{\mathrm{w}, m}\right)$, and similarly $b_{i j}$ corresponds to the action of $\beta_{i j}$. However,

$$
\beta_{i j}=\alpha_{i j}^{2}
$$

and so, at first sight, it would seem that $b_{i j}$ should be given by $A_{i j}^{2}$. However it must be recalled that the natural connection of Sect. 2 gives rise to a map from chains at $\left\{z_{r+1}^{0}, \ldots, z_{m}^{0}, w_{1}, \ldots, w_{n}\right\}=\mathbf{a}_{r}^{0}$ to those where $i$ and $j$ have been transposed, induced from $\alpha_{i}$. There is a correspondence between the chain spaces obtained by using the natural identification which exists between the spaces $Y_{\mathrm{w}, m}$ and $Y_{\sigma(\boldsymbol{w}), m}$, for any $\sigma \in S_{n}$. The matrix $A_{i j}$ is defined to be the composition of the map induced by the connection with this identification map. The square of the map induced by the connection here is precisely $b_{i j}$. However the composition of the identification maps:

and

$$
\left\{\text { chains at } \mathbf{a}_{\mathbf{r}}^{0}\right\} \leftrightarrow\left\{\text { chains at }(i j)\left(\mathbf{a}_{\mathbf{r}}^{0}\right)\right\}
$$

$$
\left.\left\{\text { chains at }(i j)\left(\mathbf{a}_{\mathbf{r}}^{0}\right)\right\} \leftrightarrow\left\{\text { chains at } \mathbf{a}_{\mathbf{r}}^{0}\right)\right\}
$$

which occur in the two applications of $A_{i j}$, leaves a residual factor of $q_{i j}$, coming from the fact that overall, $i$ and $j$ have gone round each other once in a clockwise direction. Here (ij) $\in S_{m+n}$ acts on $\mathbf{a}_{r}^{0}$ in the usual way (see Fig. 3.3), and thus (iv) is obtained.

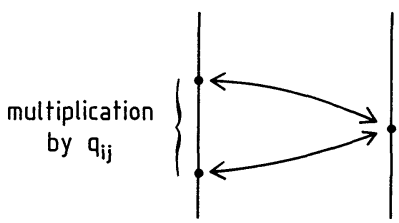

\{space of chains $\left.\left\langle\mathscr{S}_{\mathbf{a}}^{r}\right\rangle\right\}$

Fig. 3.3

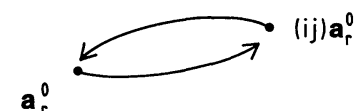

$\{\mathbf{a}\}$

It is clear that (vii), (viii), and (ix) follow directly from (i), (ii), (v), and (vi). We are now left with (i), (ii), (iii), (v), (vi). However $A_{i j}$ and $b_{i j}$ are defined in terms of the actions of the braid group, as is illustrated in Figs. 3.4 and 3.5.

Fig. 3.4

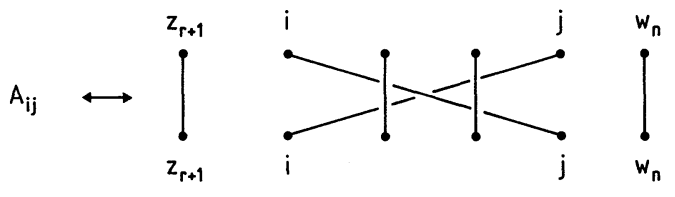

Fig. 3.5
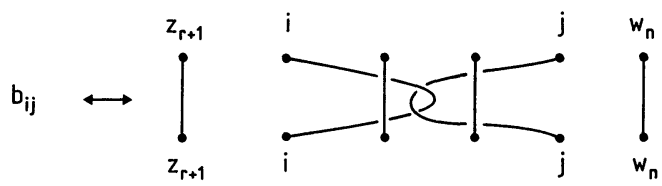

Thus we can verify all the relations by checking that the corresponding equations hold on the braid group. (The extra factors of $q_{i j}$ which may appear, due to the reason outlined above in the proof of (iv), are identical on either side for all of 
the relations considered, so that we need only consider the maps induced by the connection.) We obtain Figs. 3.6-3.9 for (i), (ii), (iii), and (iv). Finally (vi) states that for $j<\lambda<k, b_{i \lambda}$ commutes with $b_{i j}^{-1} A_{j k} b_{i j}$. This latter transformation is given by Fig. 3.10, and the commutativity of this with $b_{i \lambda}$ is given by Fig. 3.11. This equivalence follows by sliding the twist of $i$ around $\lambda$ down the diagram until it comes out at the base. Since the relations can be checked at the braid group level, they can all be derived algebraically from the braid group relations. However it is nicer to derive them geometrically!

Fig. 3.6
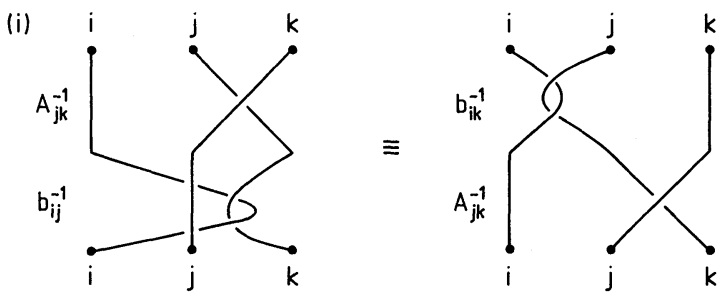

Fig. 3.7
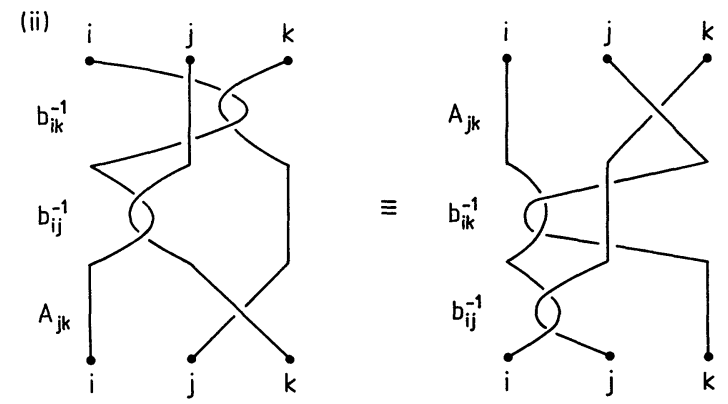

Fig. 3.8
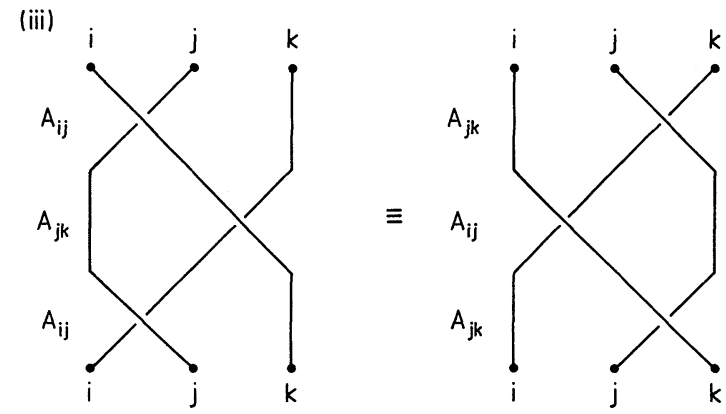

\subsection{Action of the Braid Group}

We are now in a position to derive recursion formulae for $A_{\lambda \mu}^{(r)}$ and $b_{\lambda \mu}^{(r)}$ in terms of the matrices $\left\{A_{\lambda \mu}^{(r-1)}\right\}$ and $\left\{b_{\lambda \mu}^{(r-1)}\right\}$. Such relations connect the transformation properties of embedded $r$-torii with $z_{r+1}, \ldots, z_{m}$ and $w_{1}, \ldots, w_{n}$ fixed with those of embedded $(r-1)$-torii with $z_{r}, \ldots, z_{m}, w_{1}, \ldots, w_{n}$ fixed. One can think of the space $\mathscr{S}_{\mathrm{w}}^{r}$ as equivalent to $\mathscr{S}_{w}^{m}$, where one replaces $\left\{z_{1}, \ldots, z_{m}\right\}$ by $\left\{z_{1}, \ldots, z_{r}\right\}$, and $\left\{w_{1}, \ldots, w_{n}\right\}$ by $\left\{z_{r+1}, \ldots, z_{m}, w_{1}, \ldots, w_{n}\right\}$. Passing from $\mathscr{S}_{\mathrm{w}}^{r}$ to $\mathscr{S}_{\mathrm{w}}^{r-1}$ is thus given by thinking of one of the $z$ 's (namely $z_{r}$ ) as part of the set of parameters on the base (the $w$ 's) rather than as a parameter on the fibre. 
Fig. 3.9
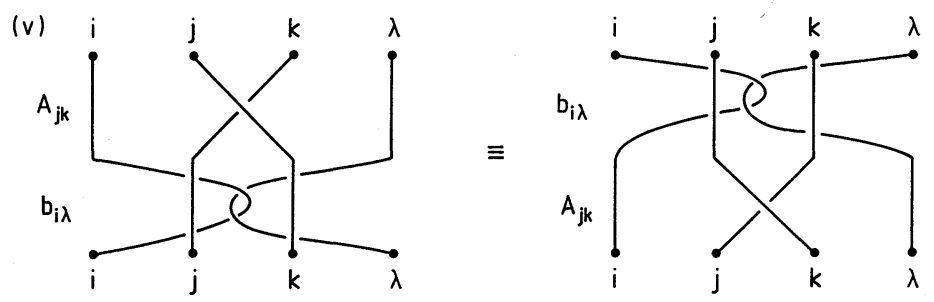

Fig. 3.10
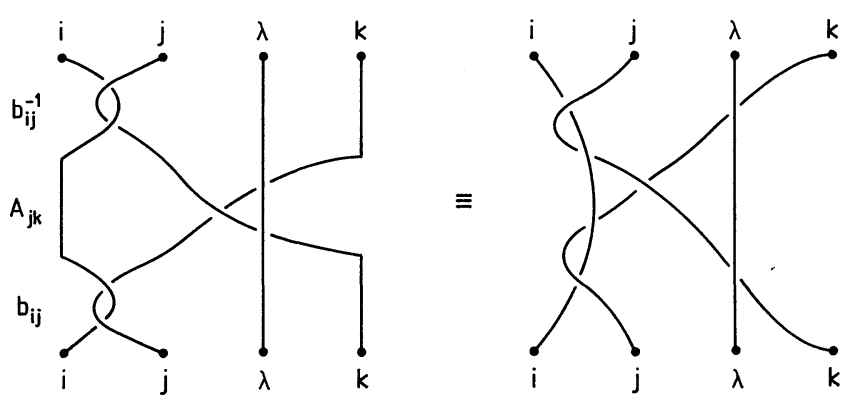

Fig. 3.11
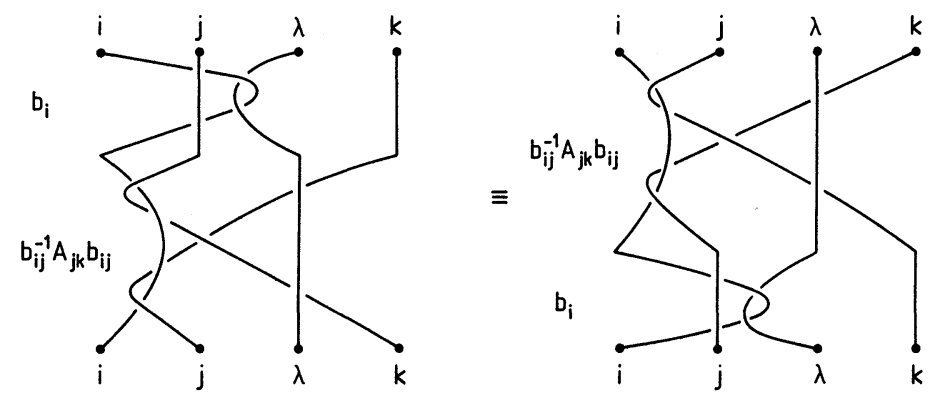

Theorem 3.4. The actions of $A_{\lambda \mu}^{(r)}$ and $b_{\lambda \mu}^{(r)}$ on $\left\langle\mathscr{T}_{w}^{r}\right\rangle$ are given in block form by the following matrices, in which the blocks are separated by the value of $\alpha_{r} \in\left\{z_{r+1}, \ldots, z_{m}\right.$, $\left.w_{1}, \ldots, w_{n}\right\}$ :

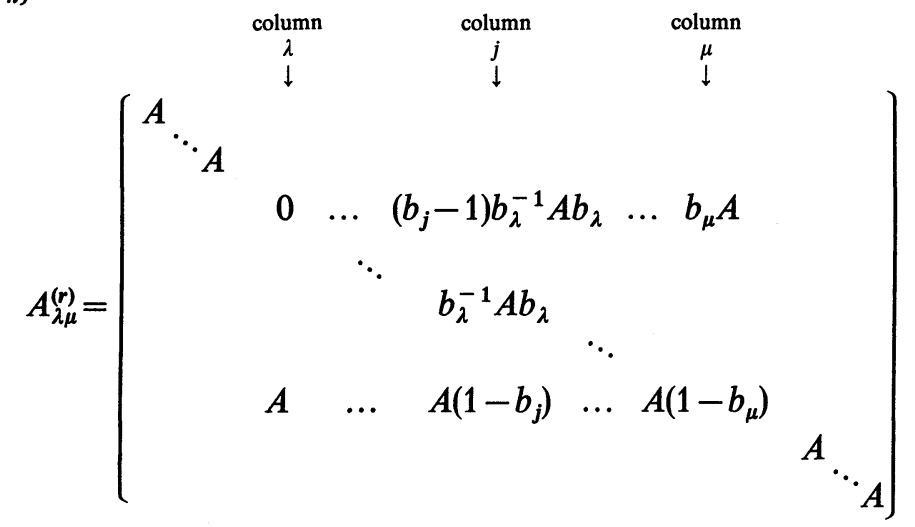


and:

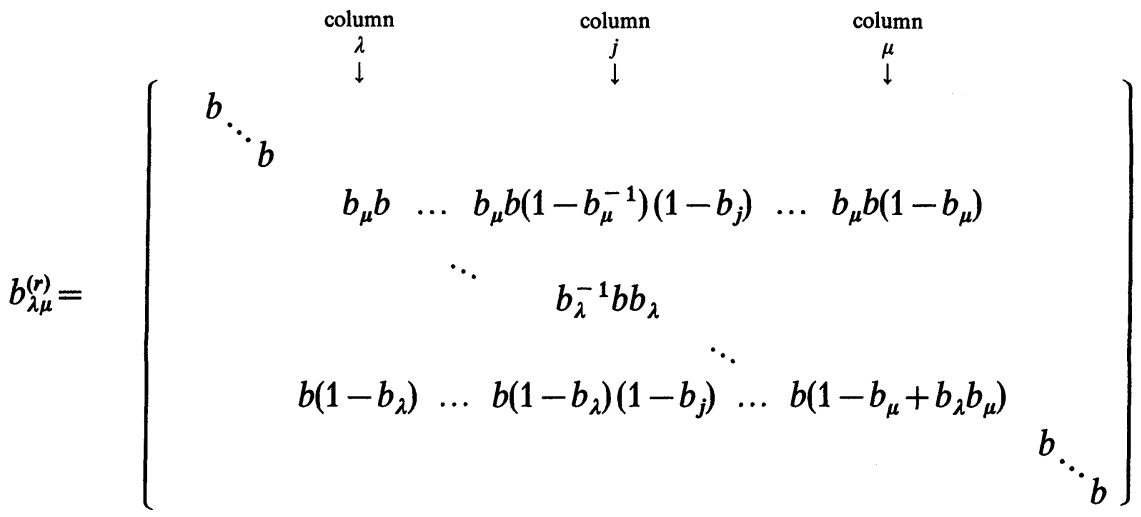

where all entries vanish except those given above; $b_{\lambda}=b_{z_{r} \lambda}^{(r-1)}, b=b_{\lambda \mu}^{(r-1)}, A=A_{\lambda \mu}^{(r-1)}$.

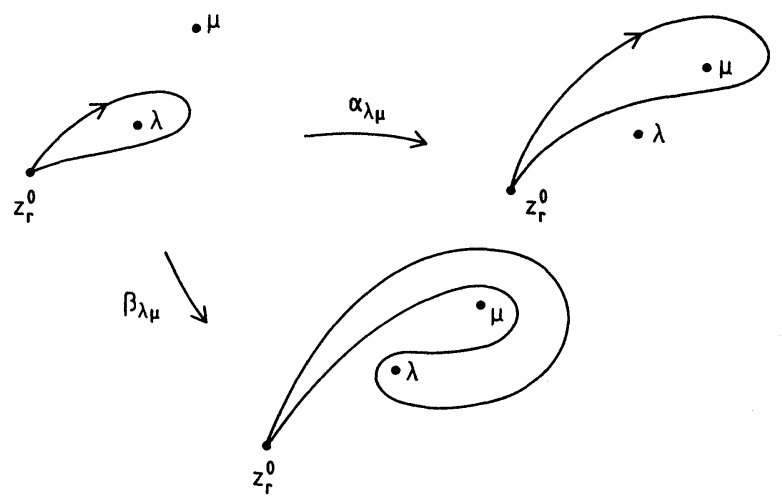

Proof. To determine the action of $A_{\lambda \mu}^{(r)}$ and $b_{\lambda \mu}^{(r)}$ on an $r$-torus $\gamma_{\alpha}$, for $\underline{\alpha} \in \mathscr{S}_{w}^{r}$, we consider separately the four cases of the different relative positions of $\alpha_{r}$ with respect to $\lambda$ and $\mu$. In each case we evaluate the actions of $A_{\lambda \mu}^{(r)}$ and $b_{\lambda \mu}^{(r)}$ on the $r$-torus $\gamma \beta \cdot \alpha_{r}$ where $\beta=\underline{\alpha}^{r-1} \in \mathscr{S}_{w}^{r-1}$, in terms of the action of the braid group on $(r-1)$ torii.

Case (i): Either $\alpha_{r}>\lambda, \mu$ or $\alpha_{r}<\lambda, \mu$.

In this case, under transposition of $\lambda$ and $\mu$, the loop $\beta_{z_{r} \alpha_{r}}$ is unchanged, and thus $\gamma_{\alpha}$ transforms to $\gamma_{\beta}$, that is:

$$
A_{\lambda \mu}^{(r)}(\underline{\alpha})=A_{\lambda \mu}^{(r-1)}(\beta) \cdot \alpha_{r} .
$$

Similarly one obtains $b_{\lambda \mu}^{(r)}(\underline{\alpha})=b_{\lambda \mu}^{(r-1)}\left(\underline{\alpha}_{r-1} \cdot \alpha_{r}\right)$.

Case (ii): $\alpha_{r}=\lambda$.

In this case the transformation given by $\alpha_{\lambda \mu}, \beta_{\lambda \mu}$ will deform the curve $\beta_{z_{r} \lambda}$ as in Fig. 3.12. To obtain the deformed curve in terms of the basis loops, we cut up the 
image loop under $\beta_{\lambda \mu}$ into three parts; see Fig. 3.13. This gives rise to an image of the $r$-torus $\gamma_{\alpha}$ which is:

$$
b_{\lambda \mu}^{(r-1)} \beta \cdot \mu+b_{z_{r} \mu}^{(r-1)} b_{\lambda \mu}^{(r-1)} \beta \cdot \lambda-b_{z_{r} \mu}^{(r-1)^{-1}} b_{z_{r} \lambda}^{(r-1)} b_{z_{r} \mu}^{(r-1)} b_{\lambda \mu}^{(r-1)} \beta \cdot \mu
$$

under $b_{\lambda \mu}^{(r)}$; and $A_{\lambda \mu}^{(r-1)} \beta \cdot \mu$ under $A_{\lambda \mu}^{(r)}$. In the notation of the theorem,

$$
\left.\begin{array}{l}
A_{\lambda \mu}^{(r)}(\underline{\alpha})=A \beta \cdot \mu \\
b_{\lambda \mu}^{(r)}(\underline{\alpha})=\left(1-b_{\mu}^{-1} b_{\lambda} b_{\mu}\right) b \beta \cdot \mu+b_{\mu} b \beta \cdot \lambda
\end{array}\right\} .
$$

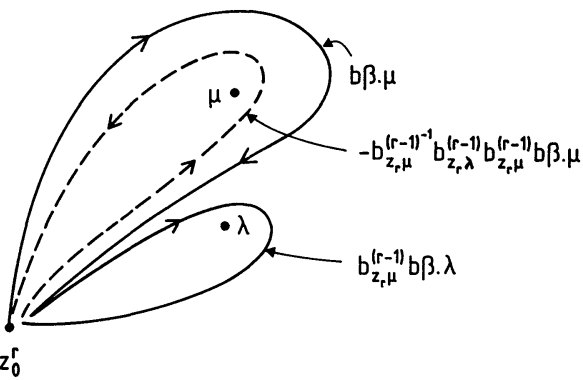

Fig. 3.13

However, by Lemma 4.3(ix), $b_{\lambda} b_{\mu} b=b_{\mu} b b_{\lambda}$, and so $\left(1-b_{\mu}^{-1} b_{\lambda} b_{\mu}\right) b=b\left(1-b_{\lambda}\right)$. This gives the $\alpha_{r}=\lambda$ columns of $A_{\lambda \mu}^{(r)}, b_{\lambda \mu}^{(r)}$ as required in the theorem.

Cases (iii), (iv): $\alpha_{r}=\mu, \lambda<\alpha_{r}<\mu$.

These cases proceed in a similar way to (i) and (ii) above. The deformed versions of the loop $\beta_{z_{r} \alpha_{r}}$ under the motions $\alpha_{\lambda \mu}, \beta_{\lambda \mu}$ are shown in Figs. 3.14 and 3.15 in these two cases. Using Lemma 4.3, the expression so obtained may'be reduced to those given in the theorem.
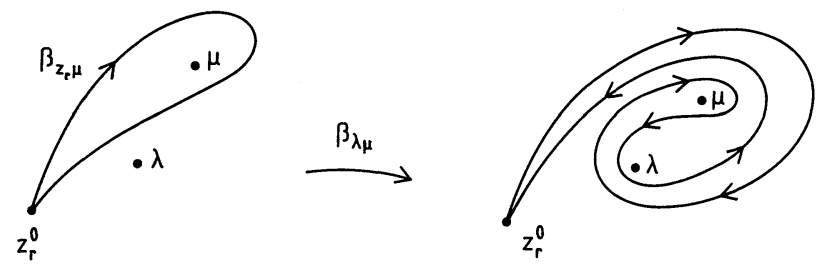

Fig. 3.14

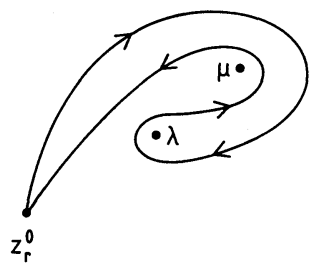

This completes the four cases required to prove the theorem. 

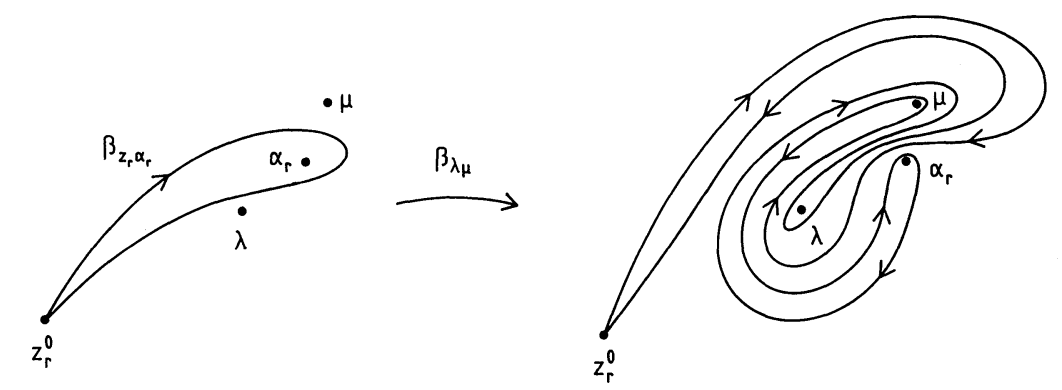

Fig. 3.15

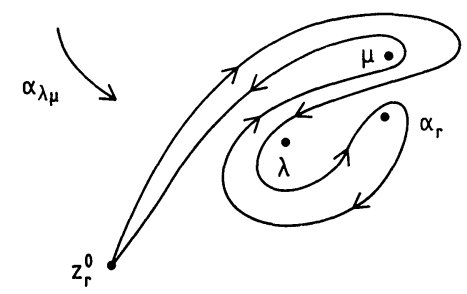

Theorem 3.4 can be used to recursively compute the matrices $A_{\lambda \mu}^{(r)}, b_{\lambda \mu}^{(r)}$ for all $z_{r+1} \leqq \lambda \leqq \mu \leqq w_{n}$ starting from the zeroth order matrices $A_{\lambda \mu}^{(0)}=1$ and $b_{\lambda \mu}^{(0)}=q_{\lambda \mu}^{-1}$. Using Theorem 3.4, it is easily seen that:

$$
\left.\begin{array}{l}
\mathbf{D}_{r}^{(r)} A_{\lambda \mu}^{(r)}=A_{\lambda \mu}^{(r-1)} \mathbf{D}_{r}^{(r)} \\
\mathbf{D}_{r}^{(r)} b_{\lambda \mu}^{(r)}=b_{\lambda \mu}^{(r-1)} \mathbf{D}_{r}^{(r)}
\end{array}\right\},
$$

where $D_{r}^{(r)}$ is given by (3.3). From (3.4), it may be observed that:

$$
\left.\begin{array}{l}
\mathbf{D}_{r}^{(m)} A_{\lambda \mu}^{(m)}=\overline{A_{\lambda \mu}^{(m-1)}} \mathbf{D}_{r}^{(m)} \\
\mathbf{D}_{r}^{(m)} b_{\lambda \mu}^{(m)}=\overline{b_{\lambda \mu}^{(m-1)}} \mathbf{D}_{r}^{(m)}
\end{array}\right\},
$$

where $\overline{A_{\lambda \mu}^{(m-1)}}$ and $\overline{b_{\lambda \mu}^{(m-1)}}$ denote the matrices obtained when $\alpha_{\lambda \mu}, \beta_{\lambda \mu}$ are applied to the space of chains $\left\langle\mathscr{S}_{w}^{m}\right\rangle$, where we replace $\left\{z_{1}, \ldots, z_{m}\right\}$ by $\left\{z_{1}, \ldots, \widehat{z}_{r}, \ldots, z_{m}\right\}$. These latter matrices thus act on a space spanned by $\underline{\alpha}=\left(\alpha_{1}, \ldots, \hat{\alpha_{r}}, \ldots, \alpha_{m}\right)$ with $\alpha_{i} \in\left\{z_{i+1}, \ldots, z_{m}, w_{1}, \ldots, w_{n}\right\}$. From (3.5) it can be seen that the matrices $A_{\lambda \mu}^{(m)}, b_{\lambda \mu}^{(m)}$ given by Theorem 3.4 preserve $\operatorname{ker}\left(\mathbf{D}_{r}^{(m)}\right)$.

Since by Lemma 3.2, the homology is given by the intersection of $\operatorname{ker}\left(\mathbf{D}_{r}\right)$ over $r=1,2, \ldots, m$, thus one may obtain the actions of $A_{\lambda \mu}^{(m)}, b_{\lambda \mu}^{(m)}$ on homology by restricting to the subspace $\operatorname{ker}\left(\mathbf{D}_{r}\right)$ at the $r^{\text {th }}$ stage of calculation. Under such a procedure, starting with $A^{(0)}, b^{(0)}$ matrices, we use Theorem 3.4 to obtain the $A^{(1)}$, $b^{(1)}$ matrices and then restrict to $\operatorname{ker}\left(\mathbf{D}_{1}\right)$. These reduced matrices are then used at the next stage, being substituted into Theorem 3.4 again. At the end of the procedure, the matrices obtained give only the actions of $A^{(m)}$ and $b^{(m)}$ matrices on homology (and not on all chains). In Sect. 4 these procedures will be illustrated in some simple cases.

\subsection{Action of the Symmetric Group}

As seen in Sect. 2, the symmetric group $S_{m}$, so long as $\mathbf{q}$ is suitably chosen, has a natural action on the homology space $H_{m}\left(Y_{\mathbf{w}, m}, \chi_{\mathbf{w}, m}(\mathbf{q})\right)$. This action is specified by 
the action of the generators $(i i+1)$ of $S_{m}$. Let $j_{i i+1}^{(r)}$ denote the action on $\left\langle\mathscr{S}_{w}^{r}\right\rangle$ of the transposition $z_{i} \leftrightarrow z_{i+1}$, where $i+1 \leqq r \leqq m$. By this action we mean the following:

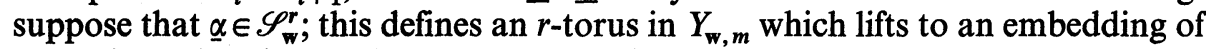
an $r$-dimensional cube in $Y_{w, m}$. Under the transposition $z_{i} \leftrightarrow z_{i+1}$, it maps to another $r$-dimensional cube in $Y_{w, m}$. However the transformed cube has base-point $\left(z_{1}^{0}, \ldots, z_{i-1}^{0}, z_{i+1}^{0}, z_{i}^{0}, \ldots, z_{m}^{0}\right)$ and this base-point does not have its imaginary parts ordered in the natural way. To correspond this with a standard $r$-chain, it is necessary to move the base-point to $\left(z_{1}^{0}, \ldots, z_{i-1}^{0}, z_{i}^{0}, z_{i+1}^{0}, \ldots, z_{m}^{0}\right)$. When this is done, the $(i+1)$-torus given by $\underline{\alpha}^{(i+1)}$ transforms to another $(i+1)$-torus, given by $j_{i i+1}^{(i+1)} \underline{\alpha}^{(i+1)}$. The whole $m$-torus $\gamma_{\alpha}$ transforms according to $j_{i i+1}^{(m)}$ where:

$$
j_{i i+1}^{(r)}=\left(\begin{array}{lll}
j_{i i+1}^{(r-1)} & \\
& \ddots & \\
& j_{i i+1}^{(r-1)}
\end{array}\right)
$$

for $r=i+2, \ldots, m$; where the blocks are separated by the value of $\alpha_{r}$. The important part of the matrix $j_{i i+1}^{(m)}$ is given by $j_{i i+1}^{(i+1)}$. The action on $\left\langle\mathscr{S}_{\mathrm{w}}^{i+1}\right\rangle$ may be given by a partitioned matrix in terms of actions on $(i-1)$-torii of the braid group, in a similar way to the recursion relations in Theorem 3.4. Using similar techniques to those used to prove Theorem 3.4, the following theorem may be obtained.

Theorem 3.5. The matrix for $j_{i i+1}^{(i+1)}$ as a partitioned matrix with blocks defined by the values of $\alpha_{i}$ and $\alpha_{i+1}$ is given by:

$$
\begin{array}{cccc}
-1) b^{\prime-1}-b_{\lambda} & \left(1-b_{\lambda}\right) b^{\prime-1}\left(b_{\lambda}^{\prime}-1\right)+b_{\lambda} b_{\lambda}^{\prime} & b_{\lambda}\left(b_{\mu}^{\prime}-1\right)+\left(1-b_{\lambda}\right) b^{\prime-1}\left(b_{\mu}^{\prime-1}-1\right) & \left(b_{\mu}-1\right) b^{\prime-1} \\
b^{\prime-1}-1 & b^{\prime-1}\left(1-b_{\lambda}^{\prime}\right)+b_{\lambda}^{\prime} & \left(b^{\prime-1}-1\right)\left(1-b_{\mu}^{\prime}\right) & 0 \\
0 & 0 & 0 & b^{\prime-1} \\
0 & 0 & 1 & 0
\end{array}
$$

where $A^{\prime}, b^{\prime}, b_{\lambda}, b_{\lambda}^{\prime}$ denote $A_{z_{i} z_{i+1}}^{(i-1)}, b_{z_{i} z_{i+1}}^{(i-1)}, b_{z_{i} \lambda}^{(i-1)}$ and $b_{z_{i+1}}^{(i-1)}$, respectively. Here the entries correspond to $z_{i+1} \lambda, \lambda \lambda$, $\mu \lambda$ and $\lambda \mu$, where $\lambda>\mu$.

\section{Examples}

In this section we will discuss the two special cases $m=1$ and $m=2$ in detail. These correspond to homology in one and two dimensions, respectively. The case $m=1$ gives rise to the simplest non-trivial braid group representation, namely the Burau representation. The case $m=2$ is the first case in which the action of the symmetric group is present. At the end of the section, the case in which the local coefficient system, $\chi_{w, m}(\mathbf{q})$ is trivial, is also discussed. The examples of this section are generalised in the next section, where we deal in detail with the case of general $m$. All the main points which arise in connection with the general case are illustrated by the examples discussed here.

\section{1. $m=1$ and the Alexander Polynomial}

When $m=1$, we have precisely one $z_{i}$, so that $\mathscr{S}_{\mathrm{w}}^{1}$ is given by $\left\{(\lambda) \mid \lambda=w_{1}, \ldots, w_{n}\right\}$. The chains thus form an $n$-dimensional space on which the braid group acts. We 
can now apply Theorem 3.4 with $r=1$, starting with $A_{\lambda \mu}^{(0)}=b_{\lambda \mu}^{(0)}=1$ and $b_{z_{1} \lambda}^{(0)}=q^{-1}$ for all $\lambda, \mu \in\left\{w_{1}, \ldots, w_{n}\right\}$. This gives rise to the following matrix for $A_{w_{i} w_{i+1}}^{(1)}$ :

$$
\left(\begin{array}{cccccc}
1 \cdot \ddots & & & & & \\
& 1 & & & & \\
& & 0 & q^{-1} & & \\
& 1 & 1-q^{-1} & & \\
& & & & 1 & \\
& & & & & \\
& & & & & 1
\end{array}\right)
$$

where the non-trivial $2 \times 2$ block occurs in the $i^{\text {th }}$ and $i+1^{\text {th }}$ rows and columns. By (3.3), the matrix for $\mathbf{D}_{1}^{(1)}$ is the $1 \times n$ matrix $\left(q^{-1}-1, \ldots, q^{-1}-1\right)$. Lemma 3.2 now gives the homology $H_{1}\left(Y_{\mathrm{w}, 1}, \chi_{\mathrm{w}, 1}(\mathbf{q})\right)$ as the subset of $\mathscr{C}_{1}$ given by $\operatorname{ker} \mathbf{D}_{1}$. Thus the homology space can be identified with the subset:

$$
\left\{\left(x_{1}, \ldots, x_{n}\right) \mid x_{1}+\ldots+x_{n}=0\right\}
$$

of $\mathscr{C}_{1}=\left\langle\mathscr{S}_{\mathrm{w}}^{1}\right\rangle \cong \mathbf{C}^{n}$. This subset is clearly preserved by the matrix $A_{w_{i} w_{i+1}}^{(1)}$ above, and thus the action of the $A^{(1)}$-matrices on homology gives rise to a representation of $B_{n}$ on an (n-1)-dimensional space. The eigenvalues of the action of $A_{w_{i} w_{i+1}}^{(1)}$ on $\mathscr{C}_{1}$ are $-q^{-1}$ and 1 (with multiplicity $n-2$ ) and hence the action of $B_{n}$ on homology factors through the Hecke algebra $H_{n}\left(q^{-1}\right)$. Its action is known as the Burau representation, $\pi_{\Lambda_{1}}$, and is important in knot theory in the context of the Alexander polynomial.

Suppose that $L$ is a link. By Alexander's Theorem [A12], $L$ can be expressed as the closure $\hat{\gamma}$ of some braid $\gamma \in B_{n}$, for suitably large $n$. In this context, the closure of a braid refers to the link obtained by joining the two sets of $n$ points to each other, as illustrated in Fig.4.1.

Fig. 4.1

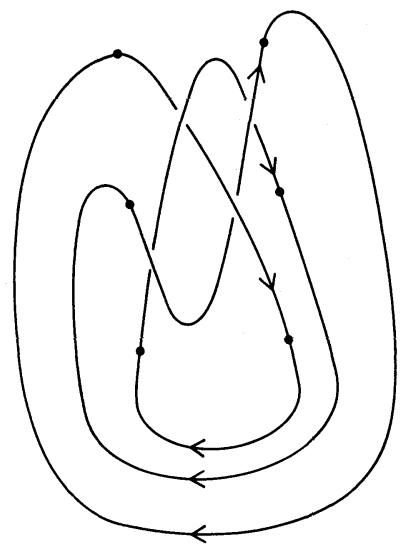

The Alexander polynomial, $\Delta_{L}$, of the link $L$, can now be defined as a polynomial in the one variable $q$ by:

$$
\Delta_{L}=\frac{\operatorname{det}\left(1-\pi_{\Lambda_{1}}(\gamma)\right)}{1+q^{-1}+\ldots+q^{1-n}}
$$


(see for example [J]). The original definition of $\Delta_{L}$, however, was given in terms of covering spaces (see [Al1], [R]). Consider the complement of the link $S^{3} \backslash L$. There is a natural map:

$$
\begin{aligned}
\pi_{1}\left(S^{3} \backslash L\right) & \rightarrow \mathbf{Z} \\
{[\Gamma] } & \mapsto(\text { the linking number of } \Gamma \text { and } L)
\end{aligned}
$$

Fig. 4.2
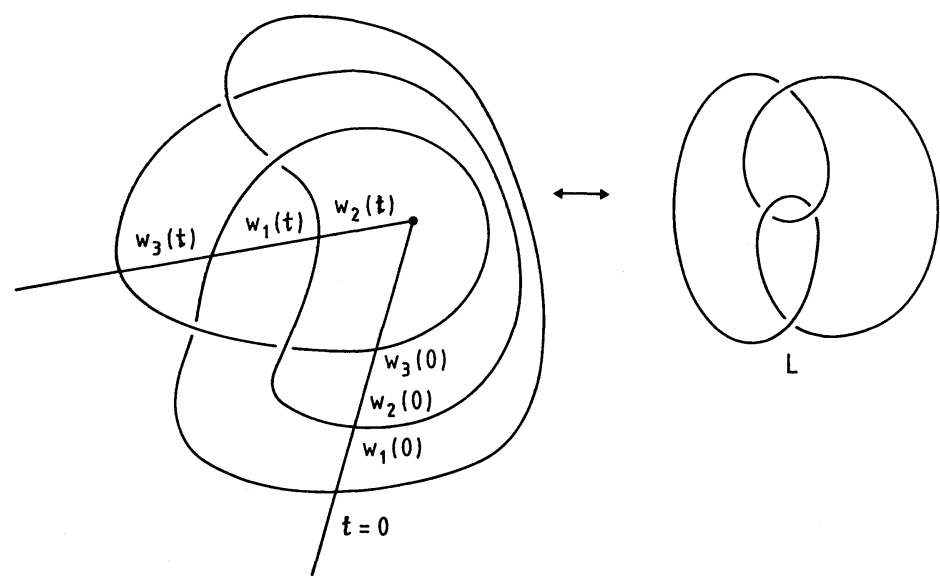

where $\Gamma$ is any closed curve in $S^{3}$, not intersecting the link $L$; and hence there is defined an infinite cyclic covering $\overline{S^{3} \backslash L}$ of $S^{3} \backslash L$. The natural action, T, on $\overline{S^{3} \backslash L}$ given by a translation in which each branch of the cover is translated into the next, induces an action on the first homology, $H_{1}\left(\overline{S^{3} \backslash L}\right)$. This homology is finite dimensional, and the characteristic polynomial of the induced action, $T_{*}$, is the Alexander polynomial. This definition of $\Delta_{L}$ makes it obvious that $\Delta_{L}$ is invariant under continuous deformations of $L$, through non-self-intersecting curves.

The braid approach to $\Delta_{L}$, as given by (4.3), can be considered as corresponding to an embedding of $L$ in $S^{1} \times S^{2}$, as opposed to an embedding in $S^{3}$. The latter approach (requiring the use of Markov's Theorem [M]) is that used in the above topological interpretation of $\Delta_{L}$. For, a braid $\gamma \in B_{n}$ is represented by a map, $\gamma: S^{1} \rightarrow \widetilde{X}_{n}$, where $\gamma(t) \in \tilde{X}_{n}$ is given by $n$ points $\left\{w_{1}(t), \ldots, w_{n}(t)\right\}$, say. The subset of $S^{1} \times S^{2}$ specified by $\left\{\left(t, w_{i}(t)\right) \mid t \in S^{1}, 1 \leqq i \leqq n\right\}$, now gives the link $L=\hat{\gamma}$, as embedded in $S^{1} \times S^{2}$. Suppose next that $\Gamma$ is a closed curve in the complement of $L$, with base-point $\left(0, z^{0}\right) \in S^{2}$. Using the correspondence between $\mathbf{C} \cup\{\infty\}$ and the Riemann sphere $S^{2}, \Gamma$ may be defined by a map:

$$
\Gamma:[0,1] \rightarrow\left(S^{1} \times S^{2}\right) \backslash L
$$

with $\Gamma(0)=\Gamma(1)=\left(0, z^{0}\right)$. Such a curve $\Gamma$ is homotopic, in $\left(S^{1} \times S^{2}\right) \backslash L$, to a combination of the curves $\Gamma_{i}(0 \leqq i \leqq n)$ defined up to homotopy by:

(i) for each $i=1,2, \ldots, n, \Gamma_{i}(t) \subseteq\{0\} \times S^{2}$ for all $t$, with the winding number of $\Gamma_{i}$, considered as embedded in $S^{2} \cong \mathbf{C} \cup\{\infty\}$, around $w_{j}(0)$ being $\delta_{i j}$;

(ii) $\Gamma_{0}(t)=\left(t, z^{0}\right)$.

Thus $\pi_{1}\left(\left(S^{1} \times S^{2}\right) \backslash L\right)$ is generated by the $(n+1)$ elements associated with $\Gamma_{0}$, $\Gamma_{1}, \ldots, \Gamma_{n}$. Under the surgery $S^{1} \times S^{2} m S^{3}$, the situation transforms so that $\Gamma_{0}$ disappears as a non-trivial generator, and $\pi_{1}\left(S^{3} \backslash L\right)$ is generated by $n$ elements, of similar form to $\Gamma_{1}, \ldots, \Gamma_{n}$. Hence, it is not surprising to find that the characteristic 
polynomial $\operatorname{det}\left(T-q^{-1}\right)$ is related to the local coefficient system on $\mathbf{C} \backslash\left\{w_{1}(0), \ldots, w_{n}(0)\right\}$ with twistings of $q^{-1}$ around each $w_{i}$. This local coefficient system can now be identified with $\chi_{w, 1}(q)$, as defined by Lemma 2.1 (see Fig. 4.2.).

The action of $B_{n}$ on $H_{1}\left(Y_{\mathrm{w}, 1}, \chi_{\mathrm{w}, 1}(\mathrm{q})\right)$ is $\pi_{A_{1}}$, as noted above, and the precise relation between the $S^{1} \times S^{2}$ and $S^{3}$ pictures is given by:

$$
\begin{aligned}
\frac{\operatorname{det}\left(1-\pi_{A_{1}}(\gamma)\right)}{1+q^{-1}+\ldots+q^{1-n}} & =\operatorname{det}\left(T_{*}-q^{-1}\right) \\
S^{1} \times S^{2} \text { picture } & \leftrightarrow S^{3} \text { picture } \\
H_{1}\left(Y_{w, 1}, \chi_{w, 1}(q)\right) \text { involved } & \leftrightarrow H_{1}\left(\widetilde{S^{3} \backslash L}\right) \text { involved } \\
\text { twist } \chi_{w_{1}}(q) & \leftrightarrow \text { parameter } q^{-1} .
\end{aligned}
$$

The interplay between $S^{1} \times S^{2}$ and $S^{3}$ also plays a major role in the dicussion of both the one-variable and generalised Jones polynomials, in Witten's theory (see [Wi] and [A]). However, this lies beyond the scope of this paper.

\subsection{The Case $m=2$ and Symmetrization}

When $m=2$, the space of chains $\mathscr{C}_{2}$ has a basis given by $\left\{\gamma_{q} \mid \underline{\alpha} \in \mathscr{S}_{w}^{2}\right\}$ where:

$$
\mathscr{S}_{\mathrm{w}}^{2}=\left\{\left(\alpha_{1}, \alpha_{2}\right) \mid \alpha_{1} \in\left\{z_{2}, w_{1}, \ldots, w_{n}\right\}, \alpha_{2} \in\left\{w_{1}, \ldots, w_{n}\right\}\right\} .
$$

We shall use the following local coefficient system $\chi_{\mathrm{w}, 2}(\mathbf{q})$ specified by the three parameters, $q_{1}, q_{2}, \alpha$ :

$$
q_{z_{i} w_{j}}=q_{i}, \quad q_{z_{1 z_{2}}}=\alpha
$$

for $i \in\{1,2\}, j \in\{1,2, \ldots, n\}$. It is necessary that $q_{z_{i} w_{j}}$ is independent of $j$, for there to be an action of the braid group $B_{n}$ on $\mathscr{C}_{2}$. For arbitrary non-zero values of the parameters, the definitions of Sect. 2 give rise to a representation of $B_{n}$. This is computed by using Theorem 3.4 and Lemma 3.2. An action of $S_{m}$ is only present if $q_{1}=q_{2}$, but we shall avoid making this specialisation until later, in order to illustrate some points that will become relevant in Sect. 5 .

Starting from the following matrices for $i=1,2$ and $w_{1} \leqq \lambda, \lambda \leqq w_{n}$ :

$$
A_{w_{\lambda} w_{\lambda+1}}^{(0)}=1, \quad b_{\lambda \mu}^{(0)}=1, \quad b_{z_{i} \lambda}^{(0)}=q_{i}^{-1}, \quad b_{z_{12}}^{(0)}=\alpha^{-1} .
$$

Theorem 3.4 may be used to obtain the matrices below for $A_{w_{\lambda} w_{\lambda+1}}^{(1)}$ and $b_{z_{2} w_{\lambda}}^{(1)}$.

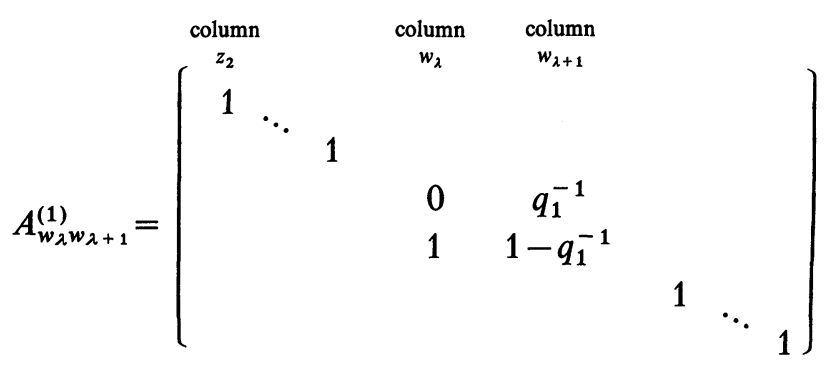




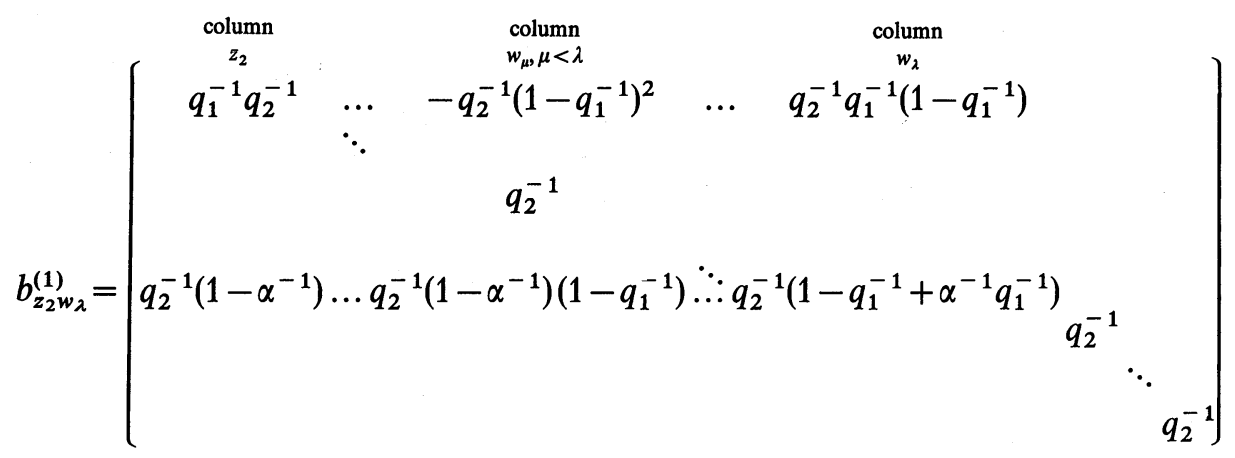

By Lemma 3.2, the homology $H_{2}\left(Y_{w, 2}, \chi_{w, 2}(\mathbf{q})\right)$ can be constructed as the subspace $\operatorname{ker}\left(\mathbf{D}_{1}\right) \cap \operatorname{ker}\left(\mathbf{D}_{2}\right)$ of the space $\left\langle\mathscr{S}_{\mathbf{w}}^{2}\right\rangle$ of chains. The action of $B_{n}$ on this subspace can be obtained by considering the matrix for $A_{w_{\lambda} w_{\lambda+1}}^{(2)}$ obtained from Theorem 3.4 using matrices $A^{(1)}, b^{(1)}$ restricted to $\operatorname{ker}\left(\mathbf{D}_{1}^{(1)}\right)$. $\mathbf{B y}(3.3), \mathbf{D}_{1}^{(1)}$ is given by the $1 \times(n+1)$ matrix:

$$
\left(\alpha^{-1}-1, q_{1}^{-1}-1, \ldots, q_{1}^{-1}-1\right),
$$

where the first element corresponds to $z_{2}$. The actions of $A_{w_{\lambda} w_{\lambda+1}}^{(1)}, b_{z_{2} w_{\lambda}}^{(1)}$ on the subspace $\operatorname{ker}\left(\mathbf{D}_{1}^{(1)}\right)$ can now be specified by $n \times n$ matrices defining the induced action on the space, $\left\{\left(0, x_{1}, \ldots, x_{n}\right)\right\} \cong\left\langle\mathscr{S}_{\mathbf{w}}^{1}\right\rangle$ under the projection:

$$
\pi_{1}^{2}:\left(x_{0}, x_{1}, \ldots, x_{n}\right) \rightarrow\left(0, x_{1}, \ldots, x_{n}\right) \text {. }
$$

This projection defines an isomorphism on $\operatorname{ker}\left(\mathbf{D}_{1}^{(1)}\right)$. The matrices obtained for $A_{w_{\lambda} w_{\lambda+1}}^{(1)}$ and $b_{z_{2} w_{\lambda}}^{(1)}$ are:

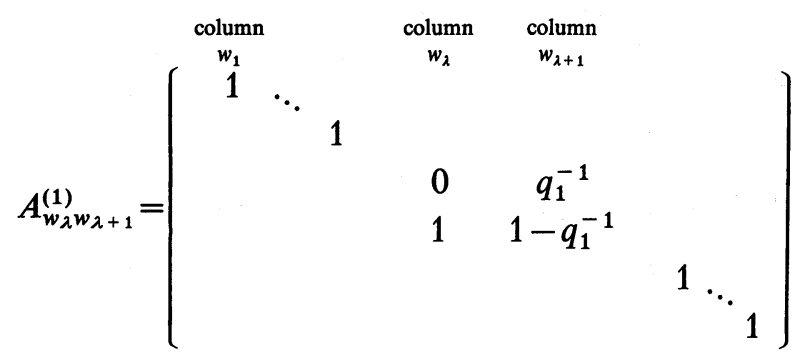

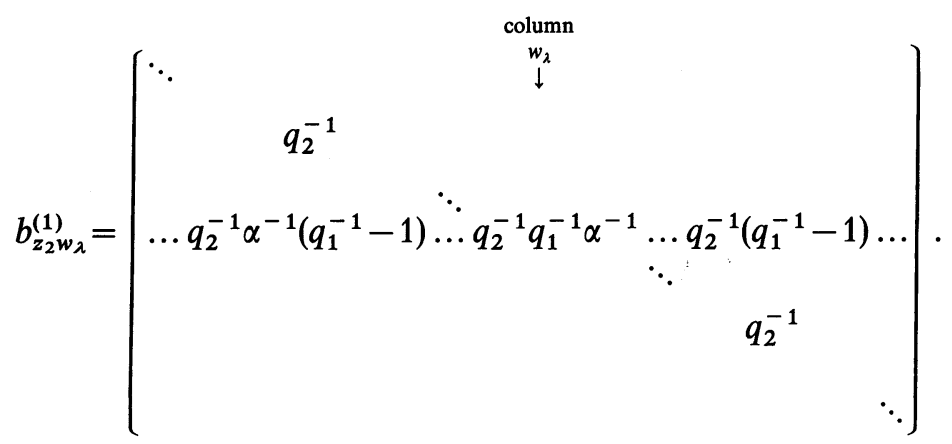


All the non-zero elements in $b_{z_{2} w_{\lambda}}^{(1)}$ occur in either the main diagonal or the $\lambda^{\text {th }}$ row.

These matrices may now be substituted into Theorem 3.4, to obtain the matrix for $A_{w_{\lambda} w_{\lambda+1}}^{(2)}$. The result obtained is shown in Fig. 4.3 below, in which $h$ denotes $-q_{1}^{-1} q_{2}^{-1} \alpha^{-1}$. We can now restrict the action to the subspace $\operatorname{ker}\left(\mathbf{D}_{2}\right)$ in order to obtain the action on homology. The matrix elements given are the only non-zero elements except for entries of 1 on the main diagonal, corresponding to basis elements $\left(w_{\lambda} w_{\mu}\right) \in \mathscr{S}_{\mathrm{w}}^{2}$, where $\lambda, \mu \in\{1,2, \ldots, n\} \backslash\{i, i+1\}$. Here $j, k$ denote elements of $\{1, \ldots, i-1\},\{i+2, \ldots, n\}$, respectively. The projection $\pi_{1}^{2}$ naturally gives a projection $\left\langle\mathscr{S}_{w}^{2}\right\rangle$, and, when this causes no confusion, the same notation, $\pi_{1}^{2}$, will be used to refer to both.

From (3.3) the matrix for $\mathbf{D}_{2}^{(2)}$ is specified by: $\mathbf{D}_{2}^{(2)}=\left(b_{z_{2} w_{1}}^{(1)}-1|\ldots| b_{z_{2} w_{n}}^{(1)}-1\right)$, while its $\lambda^{\text {th }}$ row is given by:

$$
\begin{aligned}
\sum_{\mu=1}^{n}\left(b_{z_{2} w_{\mu}}^{(1)}-1\right)_{\text {row } w_{\lambda}} \cdot w_{\mu}= & \sum_{\mu \neq \lambda}\left(\left(q_{2}^{-1}-1\right)\left(w_{\lambda} w_{\mu}\right)\right) \\
& +\sum_{j>\lambda}\left(q_{2}^{-1}\left(q_{1}^{-1}-1\right)\left(w_{j} w_{\lambda}\right)\right)+\left(q_{1}^{-1} q_{2}^{-1} \alpha^{-1}-1\right)\left(w_{\lambda} w_{\lambda}\right) \\
& +\sum_{j<\lambda}\left(q_{2}^{-1} \alpha^{-1}\left(q_{1}^{-1}-1\right)\left(w_{j} w_{\lambda}\right)\right)
\end{aligned}
$$

The subspace $\operatorname{ker}\left(\mathbf{D}_{2}^{(2)}\right)$ of $\left\langle\left(w_{\lambda} w_{\mu}\right) \mid 1 \leqq \lambda, \mu \leqq n\right\rangle$ has codimenision $n$, and is given by $n$ relations, one corresponding to each of the rows of $\mathbf{D}_{2}^{(2)}$ given above. The projection:

$$
\pi_{2}^{2}:\left\langle\left(w_{\lambda} w_{\mu}\right) \mid 1 \leqq \lambda, \mu \leqq n\right\rangle \rightarrow\left\langle\left(w_{\lambda} w_{\mu}\right) \mid 1 \leqq \lambda, \mu \leqq n, \lambda \neq \mu\right\rangle
$$

given by mapping $\left(w_{\lambda} w_{\mu}\right)$ to $\mathbf{0}$, will be an isomorphism on the restriction $\operatorname{ker}\left(\mathbf{D}_{2}^{(2)}\right)$ whenever $h \neq 0$. In this case, we can obtain an $n(n-1) \times n(n-1)$ matrix for the action of $A_{w_{i} w_{i+1}}^{(2)}$, from the induced action on $\left\langle\left(w_{\lambda} w_{\mu}\right) \mid 1 \leqq \lambda, \mu \leqq n, \lambda \neq \mu\right\rangle$ under the above projection. The matrix obtained for the action of the generator $\sigma_{i}$ of the braid group $B_{n}$ is that given in Fig. 4.4 below, where we have omitted diagonal entries corresponding to $\left(w_{\lambda} w_{\mu}\right)$ with $\lambda, \mu \in\{1,2, \ldots, n\} \backslash\{i, i+1\}, \lambda \neq \mu$, which are all 1's.

The eigenvalues of this matrix are $1,-q_{1}^{-1},-q_{2}^{-1}, \pm q_{1}^{-1} q_{2}^{-1} \alpha^{-1 / 2}$. We can only proceed further by specialising the values of $q_{1}, q_{2}, \alpha$. There is an $S_{2}$ action on the homology (and also on the space $\left\langle\mathscr{S}_{\mathrm{w}}^{2}\right\rangle$ of chains) so long as $q_{1}=q_{2}=q$, say and in this case, the matrix for $j_{12}$ as obtained from Sect. 3.4 (see Theorem 3.5) is given by the matrix of Fig. 4.5, as an action on the chain space.

The natural orthogonal projection:

$$
\left\langle\mathscr{S}_{\mathbf{w}}^{2}\right\rangle \rightarrow\left\langle\left(w_{\lambda} w_{\mu}\right) \mid 1 \leqq \lambda, \mu \leqq n, \lambda \neq \mu\right\rangle
$$

is an isomorphism on $\operatorname{ker}\left(\mathbf{D}_{1}\right) \cap \operatorname{ker}\left(\mathbf{D}_{2}\right)$ for $\alpha \neq q^{-2}$, and so there is an $n(n-1)$ $\times n(n-1)$ matrix giving the action of $j_{12}$ on homology, namely $\left(w_{\lambda} w_{\mu}\right) \mapsto \alpha\left(w_{\mu} w_{\lambda}\right)$ and $\left(w_{\mu} w_{\lambda}\right) \mapsto\left(w_{\lambda} w_{\mu}\right)$ whenever $\lambda>\mu$. This matrix has eigenvalues $\pm \alpha^{1 / 2}$, with corresponding eigenvectors $\alpha^{1 / 2}(\mu \lambda) \pm(\lambda \mu)$ for $\lambda>\mu$. Let us denote by $f_{\lambda \mu}$ the element of homology given by $\alpha^{1 / 2}(\mu \lambda)+(\lambda \mu)$, for each $\lambda>\mu$. Then the symmetric part of homology, under the action of $S_{2}$ given by $j_{12}$, is spanned by $\left\{f_{\lambda \mu} \mid \lambda>\mu\right\}$. From the matrix in Fig. 4.4, for the action of $\sigma_{i}$ on homology, we obtain: 


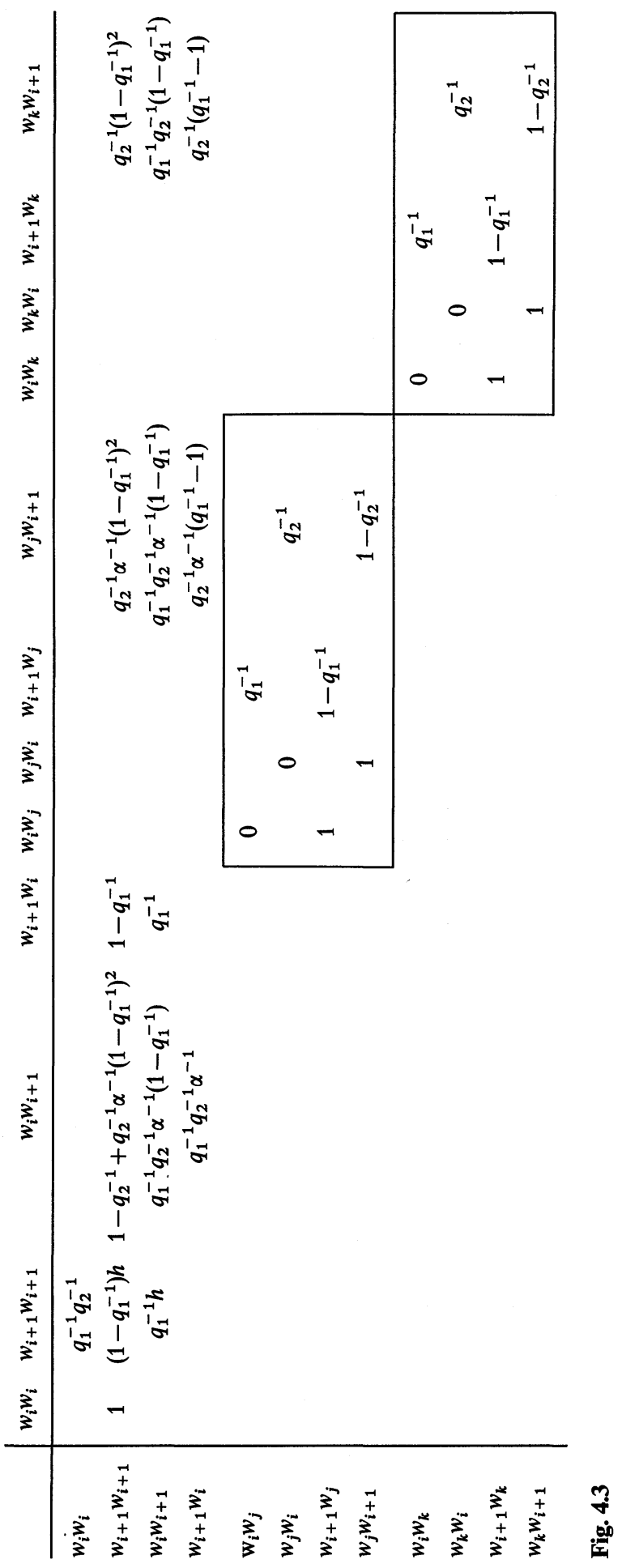



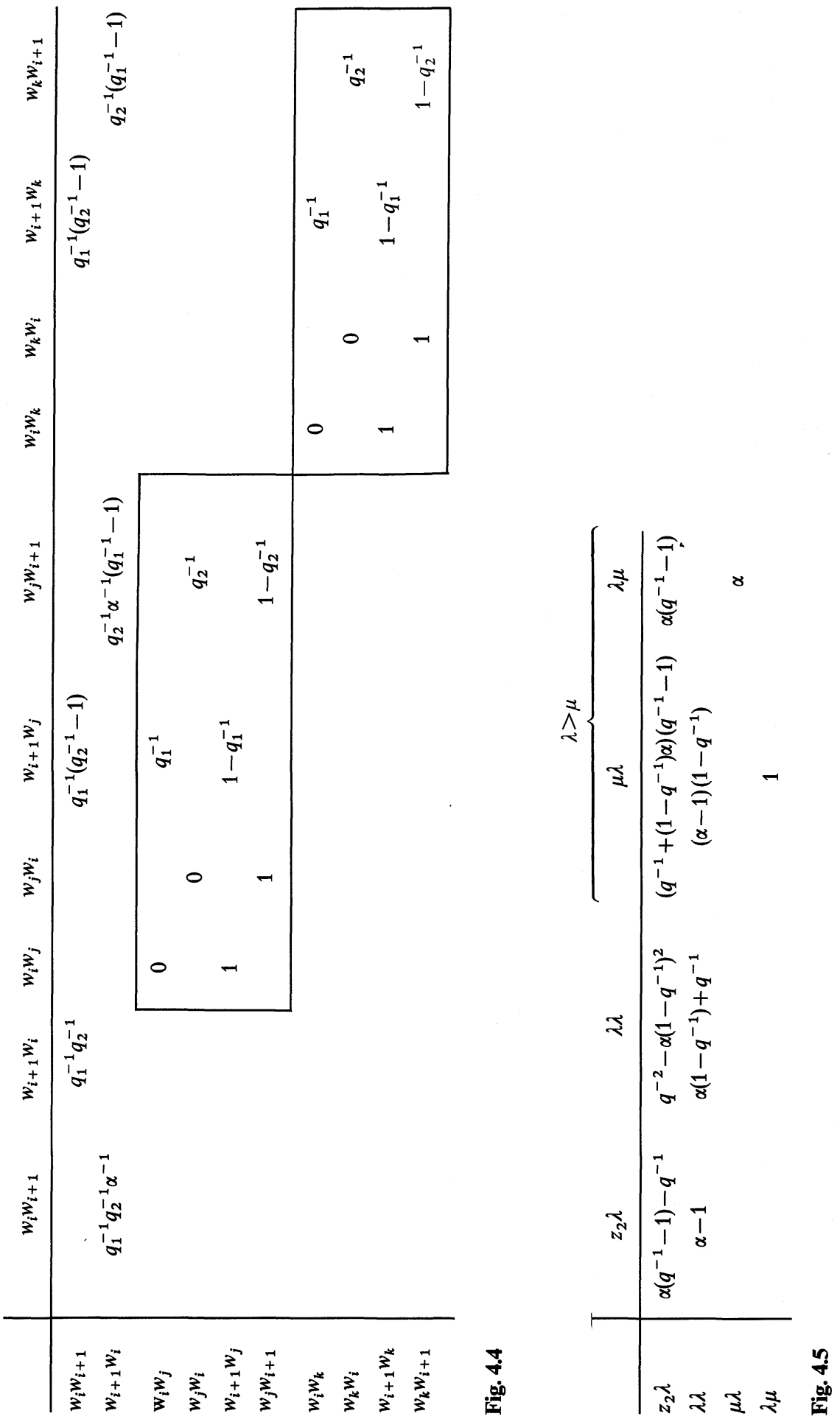
Theorem 4.1. The action of $B_{n}$ on the symmetric part of the homology $\mathrm{H}_{2}\left(Y_{\mathrm{w}, 2}\right.$, $\left.\chi_{\mathbf{w}, 2}(\mathbf{q})\right)$ is given by the matrix of Fig. 4.6 for the action of $\sigma_{i}$. This holds for all values of $\alpha$ and $q$ with $\alpha \neq q^{-2}$. Here $i, j, k$ are arbitrary with $1 \leqq j<i<k \leqq n$.

\begin{tabular}{l|ccccc} 
& $f_{i+1 i}$ & $f_{i j}$ & $f_{i+1 j}$ & $f_{k i}$ & $f_{k i+1}$ \\
\hline$f_{i+1 i}$ & $q^{-2} \alpha^{-1 / 2}$ & & $q^{-1}\left(1^{-1}-1\right) \alpha^{-1 / 2}$ & & $q^{-1}\left(q^{-1}-1\right)$ \\
$f_{i j}$ & & 0 & $q^{-1}$ & & \\
$f_{i+1 j}$ & & 1 & $1-q^{-1}$ & & \\
$f_{k i}$ & & & & 0 & $q^{-1}$ \\
Fig. 4.6 $f_{k i+1}$ & & & 1 & $1-q^{-1}$ &
\end{tabular}

On the other hand, for any $\alpha$, the symmetric part of the space of chains $\mathscr{S}_{w}^{2}$ is found to be spanned by:

$$
\left.\begin{array}{rl}
\mathbf{f}_{\lambda \mu} & =\alpha^{1 / 2}(\mu \lambda)+(\lambda \mu)+\alpha^{1 / 2}\left(q^{-1}-1\right)\left(z_{2} \lambda\right) \\
\mathbf{f}_{\lambda} & =\left(q^{-1}+\left(q^{-1}-1\right) \alpha^{1 / 2}\right)\left(z_{2} \lambda\right)+\left(1+\alpha^{1 / 2}\right)(\lambda \lambda)
\end{array}\right\} .
$$

The subspace $\left\langle\mathbf{f}_{\lambda \mu}, \mathbf{f}_{\lambda}\right\rangle$ of the span of chains intersects $\operatorname{ker}\left(\mathbf{D}_{1}\right) \cap \operatorname{ker}\left(\mathbf{D}_{2}\right)$ in a space which is isomorphic to the symmetric part of the homology. However, the map:

$$
\pi_{2}^{2} \circ \pi_{1}^{2}:\left\langle\mathscr{S}_{w}^{2}\right\rangle \rightarrow\left\langle\left(w_{\lambda} w_{\mu}\right) \mid 1 \leqq \lambda, \mu \leqq n, \lambda \neq \mu\right\rangle
$$

is an isomorphism on $\operatorname{ker}\left(\mathbf{D}_{1}\right) \cap \operatorname{ker}\left(\mathbf{D}_{2}\right)$ when $q^{-2} \neq \alpha$. In this case, $\left(\pi_{2}^{2} \circ \pi_{1}^{2}\right)^{-1}\left(w_{\lambda} w_{\mu}\right)$ defines the element of $\left\langle\mathscr{S}_{w}^{2}\right\rangle$ given by some complicated expression, namely:

$$
\left.\begin{array}{l}
(\lambda \mu)+\frac{1-q^{-1}}{q^{-2} \alpha^{-1}-1}\left((\lambda \lambda)+q^{-1}(\mu \mu)\right)+\frac{1-q^{-1}}{\left(\alpha^{-1}-1\right)\left(q^{-2} \alpha^{-1}-1\right)} \\
\quad \times\left(\left(1-q^{-1}\right)\left(z_{2} \lambda\right)+\left(q^{-2} \alpha^{-1}-1+q^{-1}-q^{-2}\right)\left(z_{2} \mu\right)\right) \text { for } \lambda>\mu \\
(\lambda \mu)+\frac{1-q^{-1}}{q^{-2} \alpha^{-1}-1}\left((\lambda \lambda)+q^{-1} \alpha^{-1}(\mu \mu)+\frac{1-q^{-1}}{\left(\alpha^{-1}-1\right)\left(q^{-2} \alpha^{-1}-1\right)}\right. \\
\quad \times\left(\left(1-q^{-1}\right)\left(z_{2} \lambda\right)+\left(q^{-1} \alpha^{-1}-1\right)\left(z_{2} \mu\right)\right) \text { for } \lambda<\mu
\end{array}\right\}
$$

In fact it can be seen that the inverse image of $\alpha^{1 / 2}(\mu \lambda)+(\lambda \mu)$ under this map is precisely:

$$
\mathbf{f}_{\lambda \mu}+\frac{1-q^{-1}}{\left(1+\alpha^{1 / 2}\right)\left(q^{-1} \alpha^{-1 / 2}-1\right)}\left[\mathbf{f}_{\lambda}+\alpha^{1 / 2} \mathbf{f}_{\mu}\right] .
$$

So, in the case $\alpha \neq q^{-2}$, when we refer to $f_{\lambda \mu}=\alpha^{1 / 2}(\mu \lambda)+(\lambda \mu)$ in the homology $H_{2}\left(Y_{\mathrm{w}, 2}, \chi_{\mathrm{w}, 2}(\mathbf{q})\right)$, as identified with $\left\langle(\lambda \mu) \mid \lambda, \mu \in\left\{w_{1}, \ldots, w_{n}\right\}, \lambda \neq \mu\right\rangle$, we are really referring to the element given by (4.8).

In the case $\alpha=q^{-2}$, all of this breaks down, since $\pi_{2}^{2}$ is no longer an isomorphism on $\operatorname{ker}\left(\mathbf{D}_{2}^{(2)}\right)$. However, inside $\operatorname{ker}\left(\mathbf{D}_{1}\right) \cap \operatorname{ker}\left(\mathbf{D}_{2}\right)$, there is a subspace of dimension $n(n-1) / 2$ given by $j_{12}=\alpha^{1 / 2}$. Unlike the case of $\alpha \neq q^{-2}$ above, there is no natural basis in terms of which the actions of $B_{n}$ and $S_{2}$ on homology can be given.

If we now switch to the dual picture, then the cohomology is given by $\mathscr{C}^{2} /\left\langle\operatorname{Im}\left(\mathbf{D}_{1}^{T}\right), \operatorname{Im}\left(\mathbf{D}_{2}^{T}\right)\right\rangle$, where $\mathscr{C}^{2}$ is a vector space dual to the space $\mathscr{C}_{2}$ of chains. 
However, in this dual picture, $\pi_{1}^{2}$ gives rise to an identification of $\mathscr{C}^{2} /\left\langle\operatorname{Im}\left(\mathrm{D}_{1}^{T}\right)\right\rangle$ with:

$$
V=\left\langle(\underline{\alpha}) \mid \alpha_{1}, \alpha_{2} \in\left\{w_{1}, \ldots, w_{n}\right\}\right\rangle .
$$

Hence, there is a representation of $B_{n}$ on $\mathscr{C}^{2} /\left\langle\operatorname{Im}\left(\mathbf{D}_{1}^{T}\right)\right\rangle$ which preserves $\operatorname{Im}\left(\mathbf{D}_{2}^{T}\right)$ and depends on the parameters $q_{1}, q_{2}$, and $\alpha$. To simplify the notation, let:

$$
V_{h}=\operatorname{Im}\left(\mathbf{D}_{2}^{T}\right)=\left\langle e_{1}, \ldots, e_{n}\right\rangle,
$$

where

$$
\begin{aligned}
e_{i}= & \left(q_{2}^{-1}-1\right) \sum_{\lambda \neq i}\left(w_{i} w_{\lambda}\right)+q_{2}^{-1}\left(q_{1}^{-1}-1\right) \sum_{k>i}\left(w_{k} w_{i}\right) \\
& +q_{2}^{-1} \alpha^{-1}\left(q_{1}^{-1}-1\right) \sum_{j<i}\left(w_{j} w_{i}\right)+\left(q_{1}^{-1} q_{2}^{-1} \alpha^{-1}-1\right)\left(w_{i} w_{i}\right) .
\end{aligned}
$$

Then

$$
W=\left\langle(\underline{\alpha}) \mid \alpha_{1}, \alpha_{2} \in\left\{w_{1}, \ldots, w_{n}\right\} ; \alpha_{1} \neq \alpha_{2}\right\rangle
$$

is clearly transverse to $V_{h}$ whenever

$$
q_{1}^{-1} q_{2}^{-1} \alpha^{-1}-1=-h \neq 0 .
$$

Let $\mathbf{A}(h)$ denote the action of $A_{w_{i} w_{i+1}}^{(2) T^{-1}}$, where $A_{w_{i} w_{i+1}}^{(2)}$ has the matrix form given in Fig. 4.3. Then $V / V_{h}$ can be identified with $W$ whenever $h \neq 0$, and so the induced action, $\mathbf{B}(h)$ of $\mathbf{A}(h)$ on $W$, gives the action of $\sigma_{i}$ on the cohomology at $\alpha^{-1}$ $=q_{1} q_{2}(1-h)$; it is given by the inverse of the transpose of the matrix of Fig. 4.4. Clearly, $\mathbf{B}(h)$ depends smoothly on $h$ near 0 , and we may denote its limit, as $h \rightarrow 0$, by $\mathbf{B}_{0}$. It may be deduced that $\mathbf{B}_{0}$ preserves the subspace $V_{0}$ of $W$, while the action of $\mathbf{B}_{0}$ on $W / V_{0}$ is identical to the restriction of $\mathbf{A}(0)$ (i.e. the action of $\sigma_{i}$ on the cohomology for $\alpha=q_{1}^{-1} q_{2}^{-1}$ ) to $W / V_{0} \subseteq V / V_{0}$. We call this action the derived action of the family $\{\mathbf{A}(h)\}$ at $h=0$; see Sect. 5 for more details. This gives an action of $\sigma_{i}$ on a space of dimension $n(n-1)$, since $\operatorname{dim} W=n^{2}, \operatorname{dim} V_{0}=n$. The action of $B_{n}$ now has two parameters, namely $q_{1}$ and $q_{2}$.

There is an action of $S_{2}$ on $\mathscr{C}^{2}$ or $H^{2}$, only when $q_{1}=q_{2}$. In this case,

$$
V_{0}=\left\langle\sum_{\lambda \neq i}\left(w_{i} w_{\lambda}\right)+q^{-1} \sum_{k>i}\left(w_{k} w_{i}\right)+q \sum_{j<i}\left(w_{j} w_{i}\right) \mid i=1,2, \ldots, n\right\rangle
$$

lies within the symmetric part of $\mathscr{C}^{2}$, since the symmetric part of $\mathscr{C}^{2}$ is spanned by:

Thus

$$
\begin{gathered}
\left(w_{i} w_{j}\right)+q\left(w_{j} w_{i}\right)=\mathbf{f}_{i j} \text { for } i>j \\
2\left(w_{i} w_{i}\right)+(1-q) \sum_{j<i}\left(w_{j} w_{i}\right)+q^{-1}\left(q^{-1}-1\right) \sum_{k>i}\left(w_{k} w_{i}\right)=\mathbf{f}_{i} \text { for all } i .
\end{gathered}
$$

$$
V_{0}=\left\langle\sum_{j<i} \mathbf{f}_{i j}+q^{-1} \sum_{k>i} \mathbf{f}_{k i} \mid i=1,2, \ldots, n\right\rangle .
$$

Under the action of $q j_{12}, \mathscr{C}^{2}$ splits into two halves of dimension $n(n+1) / 2$; and similarly $H^{2} \cong V / V_{h} \cong W$ splits into two equally sized spaces of dimension $1 / 2 n(n-1)$. However, $V_{0}$ is contained in the half with $q j_{12}=1$, and thus the dimension of the symmetric part of $W / V_{0}$ is:

$$
\frac{1}{2} n(n-1)-n=\frac{1}{2} n(n-3) \text {. }
$$

It is spanned by $\left\{\mathbf{f}_{i j} \mid i>j\right\}$ considered as elements of $W / V_{0}$, with the $n$ relations:

$$
q \sum_{j<i} \mathbf{f}_{i j}+\sum_{k>i} \mathbf{f}_{k i}=\mathbf{0} .
$$


The action of $\sigma_{i}$ on this subspace of cohomology is equivalent to an action on a quotient of homology. Alternatively, this dual action may be expressed as the action on a subspace of a space dual to $\left\{\mathbf{f}_{i j} \mid i<j\right\}$. This gives the matrix of Fig. 4.6 specialised to $\alpha=q^{-2}$, while the subspace concerned is given by the kernel of the map:

$$
\begin{aligned}
\left\langle f_{i j}\right\rangle & \rightarrow \mathbf{C}^{n} \\
f_{i j} & \mapsto\left(0, \ldots, q_{j}^{-1}, \ldots, 1, \ldots, 0\right) .
\end{aligned}
$$

It is easily seen that the matrix of Fig. 4.6 preserves this subspace, and hence there is an induced action of $B_{n}$ on this subspace. The action of $\sigma_{i}$ is given by a matrix of the same form as Fig. 4.6, except that the first row is replaced by a 1 on the diagonal and zeroes elsewhere. It is clear that this action on homology factors through $H_{n}\left(q^{-1}\right)$ since the eigenvalues of the matrix representation of the action of $\sigma_{i}$ are all $1,-q^{-1}$.

We have indicated above how this action may be obtained on either a quotient space of $\left(\mathrm{H}_{2}\right)^{S_{2}}$ at $\alpha=q^{-2}$, or as a subspace of the limiting space $\left(\mathrm{H}_{2}\right)^{S_{2}}$ for $\alpha \rightarrow q^{-2}$. The space obtained by either procedure has dimension $\frac{1}{2} n(n-1)-n=\frac{1}{2} n(n-3)$, and the action obtained factors through $H_{n}\left(q^{-1}\right)$. In terms of cohomology, this implies that a quotient action of that on the limiting space $\left(H^{2}\right)^{S_{2}}$ for $\alpha \rightarrow q^{-2}$ factors through $H_{n}(q)$. Indeed, this quotient action is given by the dual (inverse transpose) of the matrix above, in which the relations imposed are:

for $1 \leqq i \leqq n$.

$$
\sum_{j<i} \mathbf{f}_{i j}+q^{-1} \sum_{k>i} \mathbf{f}_{k i}=\mathbf{0}
$$

The work of Wenzl [We] showed how, for any Young diagram $\Lambda$ with $n$ squares, one could construct an irreducible representation $\pi_{A}$ of $H_{n}(q)$. These representations are deformations of the standard irreducible representations of $S_{n}$, as $q \rightarrow 1$. Consider the two-row Young diagram, $\Lambda_{2}$, with $n-2$ and 2 squares in its rows, as shown in Fig. 4.7; in each square, the integer indicates the hook length $l(i, j)$ as defined in $[\mathrm{J}]$. By the hook length formula the dimension of the corresponding irreducible representation of $S_{n}$ (and thus also that for $\left.H_{n}(q)\right)$ is:

$$
\frac{n !}{((\mathrm{n}-1)(\mathrm{n}-2)(\mathrm{n}-4) \ldots 1)(2.1)}=\frac{1}{2} n(n-3) \text {. }
$$

Fig. 4.7

\begin{tabular}{|c|c|c|c|c|}
\hline$n-1$ & $n-2$ & $n-4$ & $\cdots$ & 1 \\
\hline 2 & 1 & \multicolumn{3}{|l}{} \\
\cline { 1 - 3 }
\end{tabular}

The representation of $H_{n}\left(q^{-1}\right)$ on the quotient space of the symmetric part of the cohomology discussed above can now be identified by considering the limiting action as $q \rightarrow 1$. This gives a representation of $S_{n}$, and indeed the matrix for the action of $\sigma_{i}$ on $\left\langle\mathbf{f}_{i j}\right\rangle$ is precisely that of the representation of $S_{n}$ given by symmetrising the rows of $\Lambda_{2}$; that is, $\pi_{\Lambda_{2}} \oplus \pi_{\Lambda_{1}} \oplus \pi_{A_{0}}$. There are $n$ relations imposed on the f's, and they transform amongst themselves (in the case $q=1$ ) according to the natural representation of $S_{n}$ given by permuting the factors, namely $\pi_{\Lambda_{1}} \oplus \pi_{A_{0}}$. Hence the reduced action of $B_{n}$ on the quotient space is given by $\pi_{A_{2}}$. 
Theorem 4.2. There is an action of $B_{n}$ on the symmetric part of the homology $\mathrm{H}_{2}\left(Y_{\mathrm{w}, 2}, \chi_{\mathrm{w}, 2}(\mathbf{q})\right)$ for any local coefficient system $\mathbf{q}$ specified by two non-zero complex parameters $q$ and $\alpha$. When $\alpha=q^{-2}$, this action preserves an $n$-dimensional subspace of this $\frac{1}{2} n(n-1)$-dimensional space, and the quotient action induced is the irreducible representation, $\pi_{\Lambda_{2}}$, of the Hecke algebra $H_{n}\left(q^{-1}\right)$ associated with the two-row Young diagram wit $n-2$ and 2 squares in its rows.

In the dual picture, in terms of cohomology, we also have:

Theorem 4.3. Consider the family of representations of $B_{n}$ on the quotient of the chain space $\mathscr{C}^{2}$ obtained by dividing out by the boundaries $\operatorname{Im}\left(\mathbf{D}_{1}^{T}\right)$, and using the local coefficient system, $\chi$, specified by $q, \alpha$, as given in Lemma 2.1 and (2.3). Then the derived representation of this family at $\alpha=q^{-2}$, is a representation of $B_{n}$ on $a$ subspace of the cohomology $H^{2}\left(Y_{\mathrm{w}, m}, \chi\right)$. Moreover, the part of the derived representation invariant under the action of $S_{2}$ factors through $H_{n}(q)$, and is the irreducible representation $\pi_{\boldsymbol{A}_{2}}$.

\subsection{Symmetric Group Representations for $q=1$}

In this section we will discuss the case when $q=1$. In this case, the local coefficient system is trivial. Thus the $A_{\lambda \mu}^{(0)}$ and $b_{\lambda \mu}^{(0)}$ matrices are all just 1 . Theorem 3.4 allows one to compute the matrices $A_{\lambda \mu}^{(r)}, b_{\lambda \mu}^{(r)}$ for arbitrary $\lambda, \mu$, and $r$, and it is found that:

$$
b_{\lambda \mu}^{(r)}=\mathbf{I}
$$

while the action of $A_{\lambda \mu}^{(r)}$ on $\left\langle\mathscr{S}_{\mathrm{w}}^{r}\right\rangle$ has $A_{\lambda \mu}^{(r)}(\underline{\alpha})=\beta$ for $\underline{\alpha}, \beta \in \mathscr{S}_{\mathrm{w}}^{r}$, where:

$$
\beta_{i}=\left\{\begin{array}{lll}
\alpha_{i} & \text { if } & \alpha_{i} \neq \lambda, \mu \\
\mu & \text { if } & \alpha_{i}=\lambda \\
\lambda & \text { if } & \alpha_{i}=\mu .
\end{array}\right.
$$

The representation of $B_{n}$ on homology thus factors through $S_{n}$, the action of $\sigma \in S_{n}$ on $\mathscr{S}_{\mathbf{w}}^{m}$ being given by:

$$
\sigma(\underline{\alpha})=\underline{\beta},
$$

where $\beta_{i}$ is obtained from $\alpha_{i}$ by the induced action of $\sigma$ on $\left\{z_{i+1}, \ldots, z_{m}, w_{1}, \ldots, w_{n}\right\}$. The character of the representation is thus given by:

$$
\chi(\sigma)=\left(\sigma^{1}+m-1\right) \ldots\left(\sigma^{1}+1\right) \sigma^{1},
$$

where $\sigma^{r}$ is the number of cycles of order $r$ in the disjoint cycle decomposition of $\sigma$.

The formulae given in Sect. 3.2 for the matrices $D_{i}^{(r)}$ also simplify greatly. Thus, from (3.3), it is seen that $D_{i}^{(i)}=\mathbf{0}$ for all $i$. Hence $\mathbf{D}_{i}^{(r)}=\mathbf{0}$, by (3.4), and so Lemma 3.2 reduces to the trivial statement that the homology $H_{m}\left(Y_{\mathrm{w}, m}, \chi_{\mathbf{w}, m}(\mathbf{q})\right)$ can be identified with $\mathscr{C}_{m}$. The action of $S_{m}$ on the space of chains is specified in Theorem 3.5 in terms of the matrices:

$$
j_{i i+1}^{(i+1)}=\left(\begin{array}{cccc}
-A^{\prime} & A^{\prime} & & \\
& A^{\prime} & & \\
& & & A^{\prime} \\
& & A^{\prime} &
\end{array}\right),
$$


where $A^{\prime}=A_{z_{i} z_{i+1}}^{(i-1)}$ and the blocks of the above matrix are associated with the values $z_{i+1} \lambda, \lambda \lambda, \mu \lambda, \lambda \mu(\lambda>\mu)$ of the pair $\alpha_{i} \alpha_{i+1}$. The matrix gives the action on $\left\langle\mathscr{S}_{w}^{i+1}\right\rangle$, and it is extended to give the action on $\mathscr{C}_{m}$, by putting blocks of $j_{i i+1}^{(i+1)}$ down the diagonal.

However, the representation with which we are concerned here, is that on

$$
V=\left[H_{m}\left(Y_{\mathbf{w}, m}, \chi_{\mathrm{w}, m}(\mathbf{q})\right)\right]^{S_{m}},
$$

that is, the subspace of $\left\langle\mathscr{S}_{\mathrm{w}}^{m}\right\rangle$ on which $j_{i i+1}=$ id $\forall i=1,2, \ldots, m-1$. By (4.9), the subspace given by $j_{i i+1}=\mathrm{id}$ is spanned by:

$$
\left(\begin{array}{c}
0 \\
0 \\
A^{\prime} a \\
a
\end{array}\right) \text { and }\left(\begin{array}{c}
a \\
\left(A^{\prime}+1\right) a \\
0 \\
0
\end{array}\right)
$$

for arbitrary $a \in \mathscr{S}_{\mathrm{w}}^{i-1}$. The action of $A_{z_{i} z_{i+1}}^{(i-1)}=A^{\prime}$ on $\mathscr{S}_{\mathrm{w}}^{i-1}$ is the natural one, under which $z_{i}$ and $z_{i+1}$ are interchanged. Hence $V$ consists of elements $\sum_{\alpha \in \mathscr{S}_{m}}\left(A_{\alpha} \cdot \underline{\alpha}\right)$ of $\left\langle\mathscr{S}_{\mathrm{w}}^{m}\right\rangle$ such that:

(a) $A_{\alpha}=A_{\beta}$ whenever $\underline{\alpha}, \underline{\beta}$ are both vectors of distinct elements, which can be obtained from each other by exchanging $z_{i}$ and $z_{i+1}$ while, at the same time, interchanging the $i^{\text {th }}$ and $i+1^{\text {th }}$ elements, for some $i$ with $1 \leqq i<m$;

(b) all the $A_{\alpha}$ are given by well defined linear combinations (which we will not give here) of those $A_{\alpha}$ for which $\alpha_{1}, \ldots, \alpha_{m}$ are all distinct.

This implies that all the $A_{q}$ are determined by $\left\{A_{q} \mid \underline{\alpha} \in \mathscr{V}\right\}$, where $\mathscr{V}$ is a suitable subset of $\mathscr{S}_{\mathbf{w}}^{m}$ of order $(n+m-1) \ldots(n+1) n / m$ !

Example 1. Consider the case of $m=2$. Then it is clear that:

$$
\mathscr{V}=\left\{\left(w_{i} w_{j}\right) \mid 1 \leqq i<j \leqq n\right\} \cup\left\{\left(z_{2} w_{i}\right) \mid 1 \leqq i \leqq n\right\}
$$

will do. The action of $S_{n}$ here is the natural action, and splits into a direct sum of representations:

(i) the induced representation coming from the identity on $S_{2} \times S_{n-2}<S_{n}$ (of dimension $\left.\frac{1}{2} n(n-1)\right)$;

(ii) the representation of $S_{n}$ induced by the identity action of $S_{1} \times S_{n-1}<S_{n}$ (that is, the natural $n$-dimensional representation of $S_{n}$ ).

Both of these parts split into irreducible components, namely as $\pi_{\Lambda_{2}} \oplus \pi_{\Lambda_{1}} \oplus \pi_{A_{0}}$ and $\pi_{\Lambda_{1}} \oplus \pi_{A_{0}}$. Hence the total representation is $\pi_{\Lambda_{2}} \oplus 2 \pi_{\Lambda_{1}} \oplus 2 \pi_{\Lambda_{0}}$. Although it still contains $\pi_{A_{2}}$, it is by no means irreducible! The representation of $S_{n}\left(\right.$ or $\left.B_{n}\right)$ on the symmetric part of the homology has character $\chi(\sigma)=\frac{1}{2} \sigma^{1}\left(\sigma^{1}+1\right)+\sigma^{2}$ for $\sigma \in S_{n}$. This should be compared with $\chi_{\Lambda_{2}}(\sigma)=\frac{1}{2} \sigma^{1}\left(\sigma^{1}-3\right)+\sigma^{2}$.

Example 2. When $m=3$, a suitable set $\mathscr{V}$ consists of all $\underline{\alpha}=\left(\alpha_{1}, \alpha_{2}, \alpha_{3}\right)$ in $\mathscr{S}_{\mathrm{w}}^{3}$, of one of the following forms:

$$
\left(w_{i} w_{j} w_{k}\right),(i<j<k) ;\left(z_{2} w_{i} w_{j}\right),(i<j) ;\left(z_{3} w_{i} w_{j}\right),(i<j) ;\left(z_{2} z_{3} w_{i}\right) .
$$

This set has order $\frac{1}{6} n(n-1)(n-2)+2 \cdot \frac{1}{2} n(n-1)+n=\frac{1}{6} n(n+1)(n+2)$, and the representation of $S_{n}$ so obtained is $\pi_{\Lambda_{3}} \oplus 3 \pi_{\Lambda_{2}} \oplus 4 \pi_{\Lambda_{1}} \oplus 4 \pi_{\Lambda_{0}}$. It is possible to prove, in the general case, the following theorem. 
Theorem 4.4. The monodromy representation of $B_{n}$ obtained in Theorem 2.3 when $q=1$, factors through $S_{n}$, and as such, has the direct sum decomposition:

$$
\bigoplus_{k=0}^{m} A_{k} \cdot \pi_{\Lambda_{k}}
$$

where $A_{k}=\sum_{r=k}^{m}\left(\begin{array}{c}m-1 \\ r-1\end{array}\right)$ for $k>0$ and $A_{0}=A_{1}$.

Note that only two-row Young diagrams enter here. This would not be true if we expressed the representation of $B_{n}$ on the whole homology space $H_{m}\left(Y_{\mathrm{w}, m}, \chi_{\mathrm{w}, m}(q)\right)$ with $q=1$ (without restricting to the $S_{m}$-invariant part), in the form of a direct sum decomposition.

When $q$ differs from 1, but is nearby, the dimension of the homology is less than that at $q=1$, since the boundaries are non-trivial in such a case. There is thus a discontinuity in the dimension of the representation obtained at $q=1$. Similar discontinuities exist at other roots of unity, but only a finite number of roots of unity are affected for a given $m$. In all cases, however, $\pi_{\Lambda_{m}}$ occurs with multiplicity 1 , and is the major part of the representation.

\section{The General Case}

In this section we will discuss the theory for general $m$. In Sects. 5.1, 5.2, the proof of Theorem 2.3 will be completed using methods similar to those used in Sect. 4.2 in the case of $m=2$. We start by constructing the subspace of cohomology in terms of the dual basis for chains described in Sect. 3.1. The actions of $B_{n}$ and $S_{m}$ on this space are computed using Theorems 3.4, 3.5, in Sect. 5.2. The subspace of cohomology was defined in terms of a limiting procedure in Sect. 4.2, for the special case of $m=2$, and the result that was used in that case is explained in a general form in Sect. 5.3. Finally, in Sect. 5.4, there are some remarks on the comparison with Tsuchiya and Kanie's approach to Hecke algebra representations.

\subsection{Construction of a Subspace of Cohomology}

The picture we have obtained up until now is that $B_{n} \times S_{m}$ acts on $\mathscr{C}_{m}$, a space of chains. This action preserves the subspace $\operatorname{ker}(\mathbf{D}) \subseteq \mathscr{C}_{m}$, where $\mathbf{D}$ denotes the matrix:

$$
\left(\begin{array}{c}
\mathbf{D}_{1}^{(m)} \\
\vdots \\
\mathbf{D}_{m}^{(m)}
\end{array}\right) .
$$

For, $\operatorname{ker}(\mathbf{D})=\bigcap_{i=1}^{m} \operatorname{ker}\left(\mathbf{D}_{i}^{(m)}\right)$, and by Lemma 3.2, there is an isomorphism between this space and the homology, $H_{m}\left(Y_{w, m}, \chi_{w, m}(\mathbf{q})\right)$ (which we shall in future abbreviate to $H_{m}$ since the context is clear).

The duality between homology and cohomology which exists via the natural pairing:

$$
\begin{aligned}
H_{m} \times H^{m} & \rightarrow \mathbf{C} \\
(\alpha, \omega) & \mapsto \omega
\end{aligned}
$$

induces natural actions of $B_{n}$ and $S_{m}$ on cohomology. In the dual picture, we have an action of $B_{n} \times S_{m}$ on the space $\mathscr{C}^{m}$ dual to the space of chains $\mathscr{C}_{m}$, which preserves 
the subspace $\operatorname{Im}\left(\mathbf{D}^{\prime}\right) \cong \mathscr{C}^{m}$, where $\mathbf{D}^{\prime}$ corresponds to the differential map $d$, just as $\mathbf{D}$ corresponds to the boundary map $\delta$. The cohomology space $H^{m}$ is now isomorphic to $\mathscr{C}^{m} / \operatorname{Im}\left(\mathbf{D}^{\prime}\right)$. To an element, $f$, of $\mathscr{C}^{m}$ one may associate a vector:

$$
\left\{\int_{\alpha} f \mid \underline{\alpha} \in \mathscr{C}_{m}\right\}
$$

The boundary map $\delta: \mathscr{C}_{m} \rightarrow \mathscr{C}_{m-1}$ gives rise to the differential map $d: \mathscr{C}^{m-1} \rightarrow \mathscr{C}^{m}$, which is specified by the transpose of the matrix for $\delta$; that is $\mathbf{D}^{\prime}=\mathbf{D}^{\mathrm{T}}$.

We wish to define a quotient $H_{m} / W$ of homology; or equivalently a subspace of cohomology. This is done by defining a subspace $W^{\prime}$ of $\mathscr{C}^{m}$, the dual to the space of chains. The situation which we now obtain is embodied in Fig. 5.1. The subspace $W^{\prime} \cong \mathscr{C}^{m}$ gives a natural embedding $s^{\prime}$. The map $r^{\prime}$ induced by $s^{\prime}$ on $W^{\prime} / \operatorname{Im}\left(\mathbf{D}^{\prime}\right)$ gives the required subspace of $\mathscr{C}^{m} / \operatorname{Im}\left(\mathbf{D}^{\prime}\right) \cong H^{m}$, as its image. This subspace may be reached, alternatively, as the image of $j$, giving $W^{\prime} / \operatorname{Im}\left(\mathbf{D}_{*}^{\prime}\right)$ where:

$$
\mathbf{D}_{*}^{\prime}: \mathbf{D}^{\prime-1}\left(W^{\prime}\right) \rightarrow W^{\prime}
$$

is the restriction of $\mathbf{D}^{\prime}: \mathscr{C}^{m-1} \rightarrow \mathscr{C}^{m}$ to $\mathbf{D}^{\prime-1}\left(W^{\prime}\right)$.

In the dual picture, $W^{\prime}$ is dual to a quotient $\mathscr{C}_{m} / W$ of the space of chains $\mathscr{C}_{m}$, giving a quotient map $s$. Restricted to $\operatorname{ker}(\mathbf{D})$, this gives the map $r$, whose image is $\operatorname{ker}(\mathbf{D}) / W$, the required quotient of homology. Alternatively, one can obtain this result as a subspace of $\mathscr{C}_{m} / W$, using $j$; namely $\operatorname{ker}\left(\mathbf{D}^{*}\right)$ where:

$$
\mathbf{D}^{*}: \mathscr{C}_{m} / W \rightarrow \mathscr{C}_{m-1} / \mathbf{D}(W)
$$

is a quotient of $\mathbf{D}: \mathscr{C}_{m} \rightarrow \mathscr{C}_{m-1}$.

Fig. 5.1

$$
\begin{aligned}
& \operatorname{ker}(\mathbf{D}) / W \stackrel{j / r}{\stackrel{j}{r}} \operatorname{ker}(\mathbf{D}) \stackrel{\mathscr{C}_{m} / W}{\stackrel{i}{\longrightarrow}} \mathscr{C}_{m} \\
& W^{\prime} / \operatorname{Im}\left(\mathbf{D}^{\prime}\right) \stackrel{r^{\prime}}{\longrightarrow} \mathscr{C}^{m} / \operatorname{Im}\left(\mathbf{D}^{\prime}\right) \stackrel{i^{\prime}}{\longleftarrow} \mathscr{C}^{m}
\end{aligned}
$$

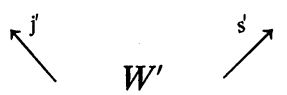

Now, to define $W^{\prime} \subseteq \mathscr{C}^{m}$, we use the natural pairing between $\mathscr{C}^{m}$ and $\mathscr{C}_{m}$, and put:

$$
W^{\prime}=\left\{f \in \mathscr{C}^{m} \mid \int_{\gamma_{\underline{\alpha}}} f=0 \text { for all } \underline{\alpha} \in \mathscr{S}_{\mathbf{w}}^{m} \backslash \mathscr{T}_{w}^{m}\right\},
$$

where $\mathscr{T}_{w}^{m}=\left\{\left(\alpha_{1}, \ldots, \alpha_{m}\right) \mid \alpha_{i} \in\left\{w_{1}, \ldots, w_{n}\right\}\right.$ for all $i$, and $\alpha_{i}$ are all distinct elements $\}$. From this definition it is clear that $\mathscr{T}_{\mathbf{w}}^{m}$, and thus also $W^{\prime}$, is trivial when $m>n$. It will be seen later in this section that $W^{\prime} / \operatorname{Im}\left(\mathbf{D}^{\prime}\right)$ is also trivial when $m>n / 2$. Dual to the basis for $\mathscr{C}_{m}$, indexed by $\mathscr{S}_{\mathrm{w}}^{m}$, there is a basis $\left\{(\underline{\alpha}) \mid \underline{\alpha} \in \mathscr{S}_{\mathrm{w}}^{m}\right\}$ for $\mathscr{C}^{m}$; and $W^{\prime}$ is spanned by those $(\underline{\alpha})$ associated with $\underline{\alpha} \in \mathscr{T}_{\mathrm{w}}^{m}$.

The action of $B_{n}$ on $H_{m}$, given by the matrices $A_{w_{i} w_{i+1}}^{(m)}$ for the action of the generator $\sigma_{i}$, corresponds in the dual picture to the action of $B_{n}$ on $H^{m}$, given by a matrix $A_{w_{i} w_{i+1}}^{(m)}$, where:

$$
\left\langle A_{w_{i} w_{i+1}}^{(m)} \mathbf{w} \mid A_{w_{i} w_{i+1}}^{(m)} \mathbf{v}\right\rangle=\langle\mathbf{w} \mid \mathbf{v}\rangle
$$

for all $\mathrm{v} \in H_{m}, \mathrm{w} \in H^{m}$, where $\langle\mid\rangle$ denotes the natural pairing between $H^{m}$ and $H_{m}$. 
Thus the matrix $A_{w_{i} w_{i+1}}^{(m)}$ is the transpose of the inverse of $A_{w_{i} w_{i+1}}^{(m)}$, giving rise to a representation of $B_{n}$ which is the dual of the representation obtained on $H_{m}$. Thus the statement of Theorem 2.3, namely that the action of $B_{n}$ on $H_{m} / W$ is an irreducible representation of $H_{n}\left(q^{-1}\right)$ is equivalent to the statement below in terms of the action on cohomology.

Theorem 5.1. There is a natural action of $B_{n} \times S_{m}$ on the subspace $\left(W^{\prime} / \operatorname{Im}\left(\mathbf{D}^{\prime}\right)\right)$ of the cohomology $H^{m}\left(Y_{\mathrm{w}, m}, \chi_{\mathrm{w}, m}(q)\right)$ and the action of $B_{n}$ on that part of the space that is totally symmetric under the action of $S_{m}$, factors through the Hecke algebra $H_{n}(q)$. Moreover this action is irreducible and corresponds to the Young diagram with two rows of lengths $n-m$ and $m$, for $m \leqq n / 2$. When $m>n / 2$, the subspace defined by $W$ is trivial.

\subsection{Actions of $B_{n}$ and $S_{m}$}

Theorems 3.4 and 3.5 may be used to compute the actions $B_{n}$ and $S_{m}$ on $W^{\prime}$. Recall the definitions of $\mathscr{S}_{\mathrm{w}}^{r}, \mathscr{T}_{\mathrm{w}}^{r}$ and $\mathscr{U}_{\mathrm{w}}^{r}$ in Sect. 3.1. There is an obvious action of the symmetric group $S_{m}$ on $\mathscr{T}_{w}^{m}$, given by:

$$
\sigma(\underline{\alpha})=\left(\alpha_{\sigma(1)} \alpha_{\sigma(2)} \ldots \alpha_{\sigma(m)}\right) .
$$

Under this action $\mathscr{T}_{w}^{m} / S_{m} \cong \mathscr{U}_{w}^{m}$. In this notation, the subspace $W^{\prime} \cong \mathscr{C}^{m}$ of Sect. 5.1 is given by:

$$
\left.\begin{array}{l}
\mathscr{C}^{m}=\left\langle(\underline{\alpha}) \mid \underline{\alpha} \in \mathscr{T}_{\mathbf{w}}^{m}\right\rangle \\
W^{\prime}=\left\langle(\underline{\alpha}) \mid \underline{\alpha} \in \mathscr{T}_{\mathbf{w}}^{m}\right\rangle
\end{array}\right\} .
$$

From Theorem 3.4, the following lemma can be deduced.

Lemma 5.2. The actions on $b_{\lambda \mu}^{(r) T}$ and $A_{\lambda \mu}^{(r)^{T}}$ on $(\underline{\alpha})$ are given by multiplication by. $q_{\lambda \mu}^{-1}$ and 1 respectively, whenever $\underline{\alpha} \in \mathscr{S}_{\mathrm{w}}^{r}$ with $\alpha_{i} \notin\left\{z_{1}, \ldots, z_{r}, \lambda, \mu\right\}$ for all $i$. Here $r$ is an integer, $1 \leqq r \leqq m$.

Definition. For any $\sigma \in S_{m}$, define $\varepsilon(\sigma) \in \mathbf{N} \cup\{0\}$ by:

$$
\varepsilon(\sigma)=\sum_{i<j} H(\sigma(i)-\sigma(j)),
$$

where the sum runs over all pairs $(i, j)$ in $\{1,2, \ldots, \mathrm{m}\}$ with $i<j$; and $H(x)$ is the Heaviside function:

$$
H(x)=\left\{\begin{array}{lll}
1 & \text { for } & x \geqq 0 \\
0 & \text { for } & x<0
\end{array}\right.
$$

Then $\varepsilon(\sigma)$ denotes the number of pairs of elements of $\{1,2, \ldots, m\}$ whose numerical order is reversed under the action of $\sigma$.

Definition. For each $\underline{\alpha} \in \mathscr{U}_{\mathrm{w}}^{r}$, define $f_{\underline{\alpha}}^{r} \equiv \sum_{\sigma \in S_{r}} q^{\varepsilon(\sigma)} \cdot(\sigma(\underline{\alpha})) \in W^{\prime}$.

Lemma 5.3. For all $\underline{\alpha} \in \mathscr{U}_{w}^{m}, f_{\underline{\alpha}}^{m}$ is preserved by the action of $S_{m}$, defined in Sect. 3.1, on $\mathscr{C}^{m}$.

Proof. It follows at once from Lemma 5.2 together with Theorem 3.5, that the action of $\sigma_{i} \in S_{m}$ on $(\underline{\alpha}) \in \mathscr{C}^{m}$ is given by:

$$
\left(q j_{i i+1}^{T}\right)(\underline{\alpha})=\left\{\begin{array}{lll}
q^{-1}\left(\alpha_{1} \ldots \alpha_{i-1} \alpha_{i+1} \alpha_{i} \ldots \alpha_{m}\right) & \text { for } & \alpha_{i}<\alpha_{i+1} \\
q\left(\alpha_{1} \ldots \alpha_{i-1} \alpha_{i+1} \alpha_{i} \ldots \alpha_{m}\right) & \text { for } & \alpha_{i}>\alpha_{i+1}
\end{array}\right.
$$


where $\alpha \in \mathscr{S}_{w}^{m}$ while $\alpha_{i}, \alpha_{i+1}$ are distinct elements of $\left\{w_{1}, \ldots, w_{n}\right\}$ and $\alpha_{j} \neq z_{i}, z_{i+1} \forall j$. The lemma now follows from the definition of $f_{\alpha}^{m}$ when it is noted that $\varepsilon(\sigma)$ satisfies the following two relations:

$$
\begin{aligned}
\varepsilon(1) & =0 ; \\
\varepsilon(\sigma \circ(i+1)) & =\varepsilon(\sigma)+\operatorname{sgn}(\sigma(i+1)-\sigma(i)) ; \quad \forall \sigma \in S_{m}, 1 \leqq i \leqq m .
\end{aligned}
$$

Lemma 5.4. The dimension of the symmetric part of $W^{\prime} \cong \mathscr{C}^{m}$ is $\frac{1}{m !} n(n-1) \ldots(n-m+1)$.

Proof. When $q$ is moved away from $1, \operatorname{dim} W^{\prime}$ cannot increase locally, since the symmetrised part can be thought of as an intersection of subspaces of $\mathscr{C}^{m}$ :

However by Lemma 5.3, $\left.\left\{f_{\alpha}^{m} \mid \underline{\alpha} \in \mathscr{U}_{\mathrm{w}}^{m}\right\} \mathbf{v} \in \mathscr{C}^{m} \mid q j_{i i+1} \mathbf{v}=\mathbf{v}\right\}$. elements of the symmetric part. The lemma follows from a dimension count for $q=1$; in this case, the action of $S_{m}$ on $W^{\prime}$ reduces to the natural action on $\left\langle\mathscr{T}^{m}\right\rangle$ given by permuting $\alpha_{i}$ s, so that the symmetrised part has dimension $(1 / m !)\left|\mathscr{T}_{\mathbf{w}}^{m}\right|$ $=\left(\begin{array}{c}n \\ m\end{array}\right)$.

Corollary 5.5. The symmetric part of $W^{\prime} \cong \mathscr{C}^{m}$ under the natural action of $S_{m}$ given by Sect: 2 is precisely $\left\langle f_{\alpha}^{m} \mid \underline{\alpha} \in \mathscr{U}_{\mathrm{w}}^{m}\right\rangle$.

In Theorem 5.1, the space on which $B_{n}$ acts is the symmetric part of the subspace $W^{\prime} / \operatorname{Im}\left(\mathbf{D}^{\prime}\right)$ of the cohomology space $\mathscr{C}^{m} / \operatorname{Im}\left(\mathbf{D}^{\prime}\right)=H^{m}$. By the above Corollary, this space is given by:

$$
\left\langle i^{\prime}\left(f_{\alpha}^{m}\right) \mid \underline{\alpha} \in \mathscr{U}_{w}^{m}\right\rangle
$$

where, for $\mathbf{v} \in \mathscr{C}^{m}, i^{\prime}(\mathbf{v})$ denotes the corresponding element of $H^{m}$. To calculate the monodromy action of $B_{n}$ on this subspace, we start by evaluating the action of $B_{n}$ on the corresponding chains $f_{\alpha}^{m}$, where $\underline{\alpha \in \mathscr{U}_{\mathrm{w}}^{m}}$, and then determine the relations which exist between these chains when they are mapped, under $i^{\prime}$, into the cohomology space. The first part is given by the following theorem.

Theorem 5.6. The generator $\sigma_{i}$ of the braid group $B_{n}$ acts on $\left\langle f_{\alpha}^{m} \mid \underline{\alpha} \in \mathscr{U}_{w}^{m}\right\rangle \leqq \mathscr{C}^{m}$ by the natural monodromy representation, according to:

$$
f_{\alpha}^{m} \rightarrow \begin{cases}f_{\alpha_{i}}^{m}+(1-q) f_{\alpha}^{m} & \text { if }\left\{w_{i}, w_{i+1}\right\} \cap\left\{\alpha_{1}, \ldots, \alpha_{m}\right\}=\left\{w_{i}\right\} \\ q f_{\alpha_{i}}^{m} & \text { if }\left\{w_{i}, w_{i+1}\right\} \cap\left\{\alpha_{1}, \ldots, \alpha_{m}\right\}=\left\{w_{i+1}\right\} \\ f_{\alpha}^{m} & \text { otherwise, }\end{cases}
$$

where $\underline{\alpha}_{i}$ denotes $\underline{\alpha}$ with any entry $w_{i}$ changed to $w_{i+1}$, and any entry $w_{i+1}$ changed to $w_{i}$.

Recall that the action of $B_{n}$ on $\mathscr{C}^{m}$ is the dual action to that on $\mathscr{C}_{m}$. The action of the generator $\sigma_{i} \in B_{n}$ on $\mathscr{C}_{m}$ is given by $A_{w_{i} w_{i+1}}^{(m)}$, and the dual action is thus given by $\left(A_{w_{i} w_{i+1}}^{(m)}\right)^{-1^{T}}$. Hence the theorem is equivalent to showing that the action of $\left(A_{w_{i} w_{i+1}}^{m)}\right)^{T}$ on the subspace of $\mathscr{C}^{m}$ spanned by $\left\{f_{\dot{\alpha}}^{m} \mid \underline{\alpha} \in \mathscr{U}_{w}^{m}\right\}$ is given by:

$$
f_{\alpha}^{m} \rightarrow \begin{cases}q^{-1} f_{\alpha_{i}}^{m} & \text { if }\left\{w_{i}, w_{i+1}\right\} \cap\left\{\alpha_{1}, \ldots, \alpha_{m}\right\}=\left\{w_{i}\right\} \\ f_{\alpha_{i}}^{m}+\left(1-q^{-1}\right) f_{\alpha}^{m} & \text { if }\left\{w_{i}, w_{i+1}\right\} \cap\left\{\alpha_{1}, \ldots, \alpha_{m}\right\}=\left\{w_{i+1}\right\} ; \\ f_{\alpha_{i}}^{m} & \text { otherwise. }\end{cases}
$$


The matrices $\left(A_{w_{i} w_{i+1}}^{(m)}\right)$ for $i=1,2, \ldots, n-1$, are given by the recursion formulae of Theorem 4.4 (Sect. 4.5), and the proof of Theorem 5.6 proceeds by applying induction on $m$ to prove many intermediate results. To avoid the necessity for using extra brackets, we shall in future use $\alpha$ to refer to the corresponding element $(\underline{\alpha})$ of $\mathscr{C}^{m}$, as well as an elements $\mathscr{S}_{w}^{m}$, so long as the context is clear.

Lemma 5.7. Whenever $\underline{\alpha} \in \mathscr{U}_{w}^{r}$,

$$
f_{\alpha}^{r}=\sum_{a=1}^{r} q^{r-a}\left(f_{\alpha(a)}^{r-1} \cdot \alpha_{a}\right),
$$

where $\underline{\alpha}(a) \in \mathscr{U}_{\mathrm{w}}^{r-1}$ is obtained by removing the $a^{\text {th }}$ element from $\underline{\alpha}$, to give $\left(\alpha_{1}, \ldots, \alpha_{a-1}\right.$, $\left.\alpha_{a+1}, \ldots, \alpha_{r}\right)$.

This lemma may be deduced from the definitions of $f_{q}^{r}$ and $\varepsilon(\sigma)$.

Lemma 5.8. Suppose that $\underline{\alpha}_{1}, \underline{\alpha}_{2} \in \mathscr{T}_{\mathrm{w}}^{r}$ are such that $\underline{\alpha}_{1}$ and $\underline{\alpha}_{2}$ differ only in the $k^{\text {th }}$ component where they are $w_{i}, w_{i+1}$ respectively, some $i, k$ with $1 \leqq k \leqq r, 1 \leqq i \leqq n-1$.

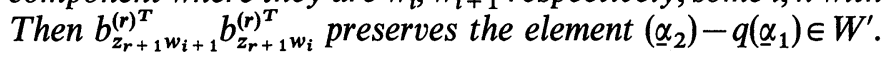

Proof. For $r=0$, the result is trivial. Assume the statement of the lemma holds for $r-1$. When $k<r$, the result follows immediately from the inductive hypothesis using Theorem 3.4 and Lemma 5.2, since $\left(\underline{\alpha}_{1}\right)_{r},\left(\underline{\alpha}_{2}\right)_{r} \notin\left\{w_{i}, w_{i+1}, z_{1}, \ldots, z_{r}\right\}$. The only case remaining is that when $k=r, \underline{\alpha}_{1}=\underline{\alpha} \cdot w_{i}$ and $\underline{\alpha}_{2}=\underline{\alpha} \cdot w_{i+1}$ for some $\underline{\alpha} \in \mathscr{T}_{w}^{r-1}$. By Theorem 3.4, since $\underline{\alpha}$ contains neither $w_{i}$ nor $w_{i+1}$,

$$
\begin{aligned}
b_{z_{r+1}}^{\left(r w_{i}\right.}\left(\underline{\alpha}_{2}-q \underline{\alpha}_{1}\right)= & b_{w_{i}}^{\prime T}(\underline{\alpha}) \cdot w_{i+1}-q\left\{\left(1-b_{z_{r+1}}^{T} b_{w_{i}}^{\prime T}(\underline{\alpha}) \cdot z_{r+1}\right.\right. \\
& +\sum_{z_{r+1}<\lambda<w_{i}}\left[\left(1-b_{\lambda}^{T}\right)\left(1-b_{z_{r+1}}^{T}\right) b_{w_{i}}^{\prime T}\right](\underline{\alpha}) \cdot \lambda \\
& \left.+\left[b_{w_{i}}^{\prime T}+b_{w_{i}}^{\prime T} b_{w_{i}}^{T}\left(b_{z_{r+1}}^{T}-1\right)\right](\underline{\alpha}) \cdot w_{i}\right\},
\end{aligned}
$$

where $b_{\lambda} \equiv b_{z_{r \lambda} \lambda}^{(r-1)}, b_{\lambda}^{\prime} \equiv b_{\lambda}^{\prime} h_{z_{r+1} \lambda}^{(r-1)}$. By Lemma 5.2 this can be reduced to:

$$
\left(q^{2}-1\right) \underline{\alpha} \cdot z_{r+1}+\left(q^{-1}-1-q\right) \underline{\alpha} \cdot w_{i}+q^{-1} \underline{\alpha} \cdot w_{i+1}+\left(q^{2}-1\right) \sum_{z_{r+1}<\lambda<w_{i}}\left(1-b_{\lambda}^{T}\right) \underline{\alpha} \cdot \lambda .
$$

When $b_{z_{r+1} w_{i+1}}^{(r)}$ is applied to this vector, using Theorem 3.4 once more, together with Lemma 5.2, one obtains:

$$
\begin{aligned}
& \left(q^{2}-1\right)\left\{q^{-2} \underline{\alpha} \cdot z_{r+1}+\left(1-q^{-1}\right) q^{-2} \underline{\alpha} \cdot w_{i+1}+q^{-2}(1-q) \sum_{z_{r+1}<j<w_{i+1}}\left(1-b_{j}^{T}\right) \underline{\alpha} \cdot w_{j}\right\} \\
& \quad+q^{-1}\left\{\left(1-q^{2}\right) q^{-1} \underline{\alpha} \cdot z_{r+1}+\left(1-q^{-1}+q\right) q^{-1} \underline{\alpha} \cdot w_{i+1}\right. \\
& \left.\quad+q^{-1}\left(1-q^{2}\right) \sum_{z_{r+1}<j<w_{i+1}}\left(1-b_{j}^{T}\right) \underline{\alpha} \cdot w_{j}\right\} \\
& \quad+\left(q^{-1}-1-q\right) q^{-1} \underline{\alpha} \cdot w_{i}+\left(q^{2}-1\right) \sum_{z_{r+1}<\lambda<w_{i}} q^{-1}\left(1-b_{\lambda}^{T}\right) \underline{\alpha} \cdot \lambda
\end{aligned}
$$

which reduces to $\underline{\alpha} \cdot w_{i+1}-q \underline{\alpha} \cdot w_{i}=\underline{\alpha}_{2}-q \underline{\alpha}_{1}$. This completes the proof of the lemma.

We now return to the proof of Theorem 5.6. As remarked above, this is complete, once (5.1) is verified for all $\underline{\alpha} \in \mathscr{U}_{w}^{r}$. For $r=1$, this follows directly from 
Theorem 3.4 applied at first order. Assume that the above action of $A_{w_{i} w_{i+1}}^{(r)}$ on $\left\langle f_{\underline{\alpha}}^{r} \mid \underline{\alpha} \in \mathscr{U}_{\mathrm{w}}^{r}\right\rangle$ holds for $r-1$. Suppose $\beta \in \mathscr{U}_{\mathrm{w}}^{r}$. By Lemma 5.7,

$$
f_{\underline{B}}^{r}=\sum_{a=1}^{r} q^{r-a}\left(f_{\underline{B}(a)}^{r-1} \cdot \beta_{a}\right) .
$$

Consider the action of $A_{w_{i} w_{i+1}}^{(r) T^{T}}$ on the terms in (5.2) separately. When $\beta$ does not contain both $w_{i}$ and $w_{i+1}$, the required transformation properties of $f_{\beta}^{r}$ at level $r$ follow, term by term in (5.2) from Theorem 3.4, Lemma 5.2 and the inductive assumption. The only case we are left with is that for which $\beta$ contains both $w_{i}$ and $w_{i+1}$. Those terms in (5.2) with $\beta_{a} \neq w_{i}, w_{i+1}$ are preserved. Thus, to show that $f_{\beta}^{r}$ is preserved, it is only necessary to show that the sum of the two terms in (5.2) corresponding to $a$ 's such that $\beta_{a}=w_{i}, w_{i+1}$, is preserved by $A_{w_{i} w_{i+1}}^{(\boldsymbol{r})^{T}}$. Since $\beta \in \mathscr{U}_{\mathrm{w}}^{r}$ then $\beta_{s}=w_{i+1}$ and $\beta_{s+1}=w_{i}$ for some $s$. Thus, it is only necessary to show that $A_{w_{i} w_{i+1}}^{(r) T}$ preserves:

$$
q f_{\underline{\underline{\beta}}(s)}^{r-1} \cdot w_{i+1}+f_{\underline{\underline{\beta}}(s+1)}^{r-1} \cdot w_{i}=q f_{\underline{\alpha}_{1}}^{r-1} \cdot w_{i+1}+f_{\underline{\alpha}_{2}}^{r} \cdot w_{i},
$$

where $\underline{\alpha}_{1}, \underline{\alpha}_{2}$ satisfy the conditions of Lemma 5.8. Since $\left\{\beta_{1}, \ldots, \beta_{s-1}, \beta_{s+2}, \ldots, \beta_{r}\right\}$ does not contain either $w_{i}$ or $w_{i+1}$, we deduce from Theorem 3.4, that:

$$
\begin{aligned}
& A_{w_{i} w_{i+1}}^{(r) T}\left(q f_{\alpha_{1}}^{r-1} \cdot w_{i+1}+f_{\alpha_{2}}^{r-1} \cdot w_{i}\right) \\
& \quad=q\left\{A^{T} f_{\alpha_{1}}^{r-1} \cdot w_{i}+\left[A^{T}\left(1-b_{w_{i+1}}^{T} b_{w_{i}}^{T} b_{w_{i+1}}^{-1}\right)\right] f_{\alpha_{1}}^{r-1} \cdot w_{i+1}\right\}+\left(A^{T} b_{w_{i+1}}^{T}\right) f_{\alpha_{2}}^{r-1} \cdot w_{i+1} \\
& \quad=q\left\{q^{-1} f_{\alpha_{2}}^{r-1} \cdot w_{i}+\left(q^{-1} f_{\alpha_{2}}^{r-1}-q A^{T} b_{w_{i+1}}^{T} b_{w_{i}}^{T} f_{\alpha_{1}}^{r-1}\right) \cdot w_{i+1}\right\}+\left(A^{T} b_{w_{i+1}}^{T} f_{\alpha_{2}}^{r-1} \cdot w_{i+1}\right.
\end{aligned}
$$

by the inductive hypothesis. (In the above we have used the simplified notation in which $A=A_{w_{i} w_{i+1}}^{(r-1)}, b_{\lambda}=b_{z_{r} \lambda}^{(r-1)}$.) Applying Lemma 5.2, we can reduce this to the form:

$$
\begin{aligned}
& f_{\alpha_{2}}^{r-1} \cdot w_{i}+\left[f_{\alpha_{2}}^{r-1}+A^{T} b_{w_{i+1}}^{T}\left(q f_{\alpha_{2}}^{r-1}-q^{2} f_{\alpha_{1}}^{r-1}\right)\right] \cdot w_{i+1} \\
& \quad=f_{\alpha_{2}}^{r-1} \cdot w_{i}+\left[f_{\alpha_{2}}^{r-1}+A^{T}\left(q f_{\alpha_{2}}^{r-1}-q^{2} f_{\alpha_{1}}^{r-1}\right)\right] \cdot w_{i+1} \quad \text { by Lemma } 5.8 \\
& \quad=f_{\alpha_{2}}^{r-1} \cdot w_{i}+q f_{\underline{\alpha}_{1}}^{r-1} \cdot w_{i+1},
\end{aligned}
$$

the last step again being a consequence of the inductive hypothesis.

The relations existing between the images of $\left\langle f_{\alpha}^{m} \mid \underline{\alpha} \in \mathscr{U}_{w}^{m}\right\rangle$ in $H^{m}$ are given by combinations of the $f_{\alpha}^{m}$ which lie in $\operatorname{Im}\left(\mathbf{D}^{\prime}\right)$. However, $\operatorname{Im}\left(\mathbf{D}_{i}^{T}\right)$ can be factored out of the space of chains $\mathscr{C}^{m}$ by setting the component of $(\underline{\alpha})$ to zero for all $\underline{\alpha} \in \mathscr{S}_{\mathrm{w}}^{m}$ with $\alpha_{i}$ $=z_{i+1}$. This is possible for $i=1,2, \ldots, m-1$, so that:

$$
\left.\mathscr{C}^{m} /\left\langle\operatorname{Im}\left(\mathbf{D}_{i}^{T}\right) \mid i=1,2, \ldots, m-1\right\rangle \cong\left\langle(\underline{\alpha}) \in \mathscr{S}_{\mathrm{w}}^{m}\right| \alpha_{i} \neq z_{i+1} \text { for } i=1,2, \ldots, m-1\right\rangle .
$$

Hence the only part of $\operatorname{Im}\left(\mathbf{D}^{\prime}\right)$ which imposes relations on $\left\{j^{\prime}\left(f_{\alpha}^{m}\right) \mid \underline{\alpha} \in \mathscr{U}_{w}^{m}\right\}$ comes from $\operatorname{Im}\left(\mathbf{D}_{m}^{T}\right)$. It can be deduced from Theorem 3.4, using Lemma 5.7, 5.2, that the following lemma holds.

Lemma 5.9. Suppose $\underline{\alpha} \in \mathscr{U}_{w}^{r}$. Then in $\mathscr{C}^{r}$ we have

$$
\left(b_{z_{m} w_{\alpha_{j}}}^{(r) T}-1\right) f_{\dot{\alpha}}^{r}=\sum_{i \neq \alpha_{\alpha^{\prime}} s}\left(f_{\left(\alpha_{1} \ldots \alpha_{j} \ldots \alpha_{r} w_{i}\right)}^{r} \cdot q^{s_{i}-j}\left(q^{-1}-1\right),\right.
$$

where $s_{i}$ is such that $\alpha_{s_{i}-1}>i>\alpha_{s_{i}}$ and $s_{i} \in\{1,2, \ldots, r\}$.

This shows, together with Lemma 5.2, that:

$$
\mathbf{D}_{m}^{T}\left(f_{\dot{\alpha}}^{m-1}\right)=\sum_{i \neq \alpha_{j}}\left(q^{-1}-1\right)\left(f_{\alpha}^{m-1} \cdot w_{i}\right)+\sum_{j=1}^{m} \sum_{i \neq \alpha_{k}^{\prime} s}\left(f_{\alpha(j) \cdot w_{i}}^{m-1} \cdot w_{\alpha_{j}}\right) q^{s_{i}-j}\left(q^{-1}-1\right) .
$$


Using Lemma 5.7, it is seen that the relations on the subset $j^{\prime}\left(W^{\prime}\right)=\left\{j^{\prime}\left(f_{\alpha}^{m}\right) \mid \underline{\alpha} \in \mathscr{U}_{w}^{m}\right\}$ of $H^{m}$ are given by:

$$
\sum_{i \neq \alpha_{j}} q^{s_{i}} f_{\alpha^{\prime} \cdot w_{i}}^{m}=0
$$

for all $\underline{\alpha} \in \mathscr{U}_{\mathrm{w}}^{m-1}$, using the usual notation for $s_{i}$. As was shown in Theorem 5.6, if we define:

$$
g_{\alpha}=\sum_{i \neq \alpha_{j}} q^{s_{i}} f_{\dot{\alpha} \cdot w_{i}}^{(m)} \in \mathscr{C}^{m}
$$

for $\underline{\alpha} \in \mathscr{U}_{w}^{m-1}$, then under $\sigma_{i} \in B_{n},\left\{g_{q}\right\}$ transforms according to:

$$
g_{\alpha} \rightarrow \begin{cases}g_{\alpha_{i}}+(1-q) g_{\alpha} & \text { if }\left\{w_{i}, w_{i+1}\right\} \cap\left\{\alpha_{1}, \ldots, \alpha_{m}\right\}=\left\{w_{i}\right\} \\ q g_{\alpha_{i}} & \text { if }\left\{w_{i}, w_{i+1}\right\} \cap\left\{\alpha_{1}, \ldots, \alpha_{m}\right\}=\left\{w_{i+1}\right\} \\ g_{\alpha} & \text { otherwise, }\end{cases}
$$

since each term in (5.4) transforms in this way.

Definition. $V^{m} \equiv\left\langle\left\{j^{\prime}\left(f_{\alpha}^{m}\right) \mid \underline{\alpha} \in \mathscr{U}_{w}^{m}\right\}\right\rangle \subseteq H^{m}$.

The action of $\sigma_{i}$ on $V^{m}$, as defined in Sect. 2, is the quotient of an action which factors through the Hecke algebra $H_{n}(q)$, as is given in Theorem 5.6. Relations (5.3) shows that this quotient is by another Hecke algebra representation, in which $m$ is replaced by $m-1$. The dimension of $V^{m}$ is:

$$
\left|\mathscr{U}_{\mathrm{w}}^{m}\right|-\left|\mathscr{U}_{\mathrm{w}}^{m-1}\right|=\left(\begin{array}{c}
n \\
m
\end{array}\right)-\left(\begin{array}{c}
n \\
m-1
\end{array}\right),
$$

since there are $\left|\mathscr{U}_{w}^{m-1}\right|$ relations satisfied by the spanning set $\left\{j^{\prime}\left(f_{\alpha}^{m}\right) \mid \underline{\alpha} \in \mathscr{U}_{w}^{m}\right\}$ of $V_{m}$ (see (5.3)). The hook length formula [J] gives the dimension of the Hecke algebra representation $\pi_{\Lambda_{m}}$, where $\Lambda_{m}$ is the two-row Young diagram with rows of length $n-m$ and $m$, as:

$$
\begin{aligned}
& \frac{n !}{(n-m+1) \ldots(n-2 m+2)(n-2 m) \ldots 1 . m !} \\
& =\frac{1}{m !} n(n-1) \ldots(n-m+2)(n-2 m+1) \\
& =\left(\begin{array}{l}
n \\
m
\end{array}\right)-\left(\begin{array}{c}
n \\
m-1
\end{array}\right) \\
& =\operatorname{dim} V^{m} .
\end{aligned}
$$

\begin{tabular}{|c|c|c|c|c|c|}
\hline$n-m+1$ & $\cdots$ & $n-2 m+2$ & $n-2 m$ & $\cdots$ & 1 \\
\hline$m$ & $\cdots$ & 1 & &
\end{tabular}

Fig. 5.2

The Hecke algebra representation given in Theorem 5.6 corresponds to that obtained by symmetrising along the rows in $\Lambda_{m}$, but not anti-symmetrising down the columns. As in the case of the symmetric group $S_{n}$, such a representation of the Hecke algebra has character:

$$
\chi_{\Lambda_{m}}+\ldots+\chi_{\Lambda_{1}}+\chi_{\Lambda_{0}}
$$


where $\chi_{A_{i}}$ is the character of the irreducible representation of $H_{n}(q)$ corresponding to the Young diagram $\Lambda_{i}$. However $V^{m}$ is the quotient of $\left\langle\left(f_{\alpha}^{m}\right) \mid \underline{\alpha} \in \mathscr{U}_{\mathrm{w}}^{m}\right\rangle$ by $\left\langle g_{q} \mid \alpha \in \mathscr{U}_{\mathrm{w}}^{m-1}\right\rangle$, and the action of $B_{n}$ on $\left\langle g_{\alpha}\right\rangle$ also factors through $H_{n}(q)$, with character $\chi_{A_{m-1}}+\ldots+\chi_{A_{0}}$. The character of the action of $B_{n}$ on $V^{m}$ is thus:

$$
\left(\chi_{\Lambda_{m}}+\ldots+\chi_{\Lambda_{0}}\right)-\left(\chi_{\Lambda_{m-1}}+\ldots+\chi_{\Lambda_{0}}\right)=\chi_{\Lambda_{m}} \text {. }
$$

Going back to Wenzl's definition of the representation $\pi_{A_{m}}$, it is easy to see that the action on $V^{m}$ of $B_{n}$ is precisely that of $\pi_{A_{m}}$, and the basis that $\mathscr{U}_{w}^{m}$ supplies at the level of chains, is a natural basis for this action. Hence Theorem 5.1 is proved.

\subsection{Selection of Subspace by Limiting Procedure}

In this section we shall discuss a procedure by which a subrepresentation may be obtained from a suitable family of quotient represenations. The subrepresentation obtained will be called the derived representation of the family. This technique was used in Sect. 4.2 to pick out the representation $\pi_{A_{2}}$ of $H_{n}(q)$ from the family of actions of $B_{n}$ on cohomology with parameters $q$ and $\alpha$, where $q$ is fixed and $\alpha$ is varied around $q^{-2}$.

Suppose $M$ is a smooth manifold and $V$ is a vector space. Let Grass $(n, V)$ denote the set of all subspaces of $V$ of dimension $n$. The family of quotient actions is given by a map A : $M \rightarrow \operatorname{End}(V)$ together with a family of subspaces of $V$ preserved by $\mathbf{A}$, specified by $f: M \rightarrow \operatorname{Grass}(n, V)$. This family is parametrised by points on $M$, and we wish to construct an action on a subspace of $V / f\left(x_{0}\right)$, where $x_{0} \in M$ is a chosen point.

Definition. A map $f: M \rightarrow \operatorname{Grass}(n, V)$ will be said to be non-degenerate at a point $x \in M$, if the derivative $d f_{x}: T_{x} M \rightarrow T_{f(x)} \operatorname{Grass}(n, V)$, when considered as a map:

has maximal rank.

$$
D f_{x}: T_{x} M \otimes f(x) \rightarrow V / f(x)
$$

The simplest case of a limiting lemma occurs with a one-parameter family of quotient actions $\mathrm{A}(h)$ on $V$ preserving $V_{h} \subseteq V$. Suppose that $W$ is chosen so that for all sufficiently small $\delta$ :

(i) $W, V_{h}$ are transverse in $V$ for $0<|h|<\delta$, and $W$ is a maximal space satisfying this condition;

(ii) $V_{0} \subseteq W \subseteq V$.

Then we have the following lemma.

Lemma 5.10. Assume that $\mathbf{A}(h), V_{h}, W, V$ are defined as described above; while $\left\{V_{h}\right\}$ is non-degenerate at $h=0$. Let $\mathbf{B}(h)$ be the induced action of $\mathbf{A}(h)$ on $W \cong V / V_{h}$, for $0<|h|<\delta$ and assume that the limit $\mathbf{B}_{0}=\lim _{h \rightarrow 0} \mathbf{B}(h)$ exists. Then:

(a) $\mathbf{B}_{0}$ preserves $V_{0}$;

(b) $\mathbf{A}(0)$ preserves $W$;

(c) the quotient action of $\mathbf{B}_{0}$ on $W / V_{0}$, and the subaction of $\mathbf{A}(0)$ on $W / V_{0} \subseteq V / V_{0}$ are identical.

We will refer to the action of $\mathbf{B}_{0}$ on $W / V_{0}$ as the derived action of the family $\{\mathbf{A}(h)\}$ at $h=0$. By part (c) of the above lemma, it is identical to the sub-action of A(0) on $W / V_{0} \subseteq V / V_{0}$. As was observed during the proof of Lemma 5.10, the existence of the limit $\mathbf{B}_{0}$ is equivalent to (b) in that lemma. This result may be generalised to multi-parameter families of linear transformations. 
Theorem 5.11. Suppose that $\mathbf{A}: M \rightarrow \operatorname{End}(V)$ is a smooth family of linear transformations on $V$, and that

$$
f: M \rightarrow \operatorname{Grass}(n, V)
$$

defines a corresponding smooth family of subspaces of $V$ which are preserved by $\mathbf{A}$. That is, $\mathbf{A}(x) f(x) \subseteq f(x)$. Assume that $f$ is non-degenerate at $x_{0} \in M$, and $W \cong V$ is a subspace such that:

(i) $f\left(x_{0}\right) \subseteq W, \mathbf{A}\left(x_{0}\right)$ preserves $W$;

(ii) $W / f\left(x_{0}\right)$ is transverse to $\operatorname{Im}\left(D f_{x_{0}}\right)$;

(iii) $W$ is maximal satisfying (ii).

Then the family of actions $\mathbf{A}(y)$ (for $\left.y \in M \backslash\left\{x_{0}\right\}\right)$ on the quotients $V / f(y)$ defines a derived action $\mathbf{B}_{0}$ on $W / f\left(x_{0}\right)$. Furthermore, this action can be obtained in either of the following ways:

(a) the restriction of the quotient action of $\mathbf{A}\left(x_{0}\right)$ on $V / f\left(x_{0}\right)$ to $W / f\left(x_{0}\right)$;

(b) the quotient of a limiting action on $W$.

Proof. The main idea of the proof is to break the family up so as to consider only a one-dimensional variation at any one time; Lemma 5.10 may then be applied repeatedly. Suppose $x_{1}, \ldots, x_{k}$ are local coordinates on $M$ near $x_{0}$. Define a sequence of spaces $W_{i} \subseteq V$ for $i=0,1, \ldots, k$ as follows. For each $i, W_{i}$ depends on the parameters $x_{i+1}, \ldots, x_{k}$, and satisfies the conditions:

( $\alpha) W_{i-1}\left(x_{i}, \ldots, x_{k}\right) / f\left(0, \ldots, 0, x_{i}, \ldots, x_{k}\right) \cong W_{i}\left(x_{i+1}, \ldots, x_{k}\right)$ for $x_{j} \neq 0$ sufficiently small;

(B) $f\left(0, \ldots, 0,0, x_{i+1}, \ldots, x_{k}\right) \subseteq W_{i}\left(x_{i+1}, \ldots, x_{k}\right)$.

We start with the initial space $W_{0}=V$, and then $W_{k}=W$ without loss of generality.

At each stage, we suppose that $\{\mathbf{A}(x)\}$ has induced an action:

$$
\mathbf{B}_{(i-1)}\left(x_{i}, \ldots, x_{k}\right) \in \operatorname{End}\left(W_{i-1}\left(x_{i}, \ldots, x_{k}\right)\right)
$$

preserving the subspace $f\left(0, \ldots, 0, x_{i}, \ldots, x_{k}\right)$, where for $i=1, \mathbf{B}_{(0)}=\mathbf{A}$. By Lemma 5.10, this induces an action $\mathbf{B}_{(i)}\left(x_{i+1}, \ldots, x_{k}\right)$ on $W_{i}\left(x_{i+1}, \ldots, x_{k}\right)$, where $x_{i+1}, \ldots, x_{k}$ are considered fixed and $x_{i}$ replaces the parameter $h$. This action corresponds to that of $\mathbf{B}_{0}$ on $W$ in Lemma 5.10. By Lemma 5.10(a), the limiting action $\mathbf{B}_{(i)}\left(x_{i+1}, \ldots, x_{k}\right)$ preserves the subspace:

$$
f\left(0, \ldots, 0, x_{i}, \ldots, x_{k}\right) \subseteq W_{i}\left(x_{i+1}, \ldots, x_{k}\right) .
$$

To sum up, we have here applied Lemma 5.10, with:

$$
\begin{aligned}
\mathbf{A}(h) & \rightarrow \mathbf{B}_{(i-1)}\left(x_{i}, \ldots, x_{k}\right) \\
W & \rightarrow W_{i}\left(x_{i+1}, \ldots, x_{k}\right) \\
h & \rightarrow x_{i} \\
\mathbf{B}_{0} & \rightarrow \mathbf{B}_{(i)}\left(x_{i+1}, \ldots, x_{k}\right) \\
V & \rightarrow W_{i-1}\left(x_{i}, \ldots, x_{k}\right) \\
V_{h} & \rightarrow f\left(0, \ldots, 0, x_{i}, \ldots, x_{k}\right) .
\end{aligned}
$$

The spaces $W_{i}$ can be chosen to be independent of $x_{i+1}, \ldots, x_{k}$ for sufficiently small $|\mathbf{x}| \neq 0$, and thus, at the $k^{\text {th }}$ stage, we observe that $\mathbf{B}_{(k)} \in \operatorname{End}\left(W_{k}\right)$ preserves $f(0, \ldots, 0) \subseteq W_{k}$. Thus in the notation of the theorem, $W=W_{k}$ and $\mathbf{B}_{0}$ is the quotient action of $\mathbf{B}_{(k)}$ on $W / f\left(x_{0}\right)$. By applying part (c) of Lemma 5.10 at each stage, it may be seen that $\mathbf{B}_{0}$ can also be obtained as the reduced action of $\mathbf{A}\left(x_{0}\right)$ on the subspace $W / f\left(x_{0}\right) \subseteq V / f\left(x_{0}\right)$. This completes the proof of the theorem. 
This theorem should be thought of as involving the transposition of the operations of dividing out invariant spaces, and taking a limit. Thus $\mathbf{B}_{(k)}$ should be considered as being obtained by first dividing out the invariant space $f(x)$ from the action of $\mathbf{A}(x)$ on $V$, and then taking the limit $x \rightarrow x_{0}$. If instead we take the limit $x \rightarrow x_{0}$ first, we obtain an action of $\mathbf{A}\left(x_{0}\right)$ on $V$ fixing $f\left(x_{0}\right)$, and dividing by this invariant space gives an action on $V / f\left(x_{0}\right)$. The theorem states that one obtains identical actions on the reduced space $W / f\left(x_{0}\right)$, namely the derived action, if one takes either

(i) a quotient of the action obtained by first dividing out invariant spaces and then taking the limit; or

(ii) a restriction of the action obtained by taking the limit first.

The space $W / f\left(x_{0}\right)$ is isomorphic to $V /\left\langle\operatorname{Im}\left(D f_{x_{0}}\right), f\left(x_{0}\right)\right\rangle$, and so should be thought of as being constructed from $V$ by removing the subspace spanned by the first order variation of vectors in the subspaces $f(x)$ with $x$ close to $x_{0}$. That is, if

$$
x:(-\varepsilon, \varepsilon) \rightarrow M \text { and } \mathbf{v}:(-\varepsilon, \varepsilon) \rightarrow V
$$

while $\mathbf{v}(t) \in f(x(t)) \forall t$ and $x(0)=x_{0}$, then $d \mathbf{v} /\left.d t\right|_{t=0}$ lies in $\left\langle\operatorname{Im}\left(D f_{x_{0}}\right), f\left(x_{0}\right)\right\rangle$. Indeed, the span of all such vectors $\mathbf{v}^{\prime}(0)$ is precisely the subspace $\left\langle\operatorname{Im}\left(D f_{x_{0}}\right), f\left(x_{0}\right)\right\rangle$ of $V$.

The monodromy representation of $B_{n}$ defined in Sect. 2 is well defined for any local coefficient system $\chi_{w, m}(\mathbf{q})$, where $q$ satisfies (2.1). That is, for any non-zero complex numbers $q_{i}, \alpha_{i j}(1 \leqq i, j \leqq m, i<j)$, a monodromy representation of $B_{n}$ comes from $q$ defined by:

$$
q_{z_{i} z_{j}}=\alpha_{i j}, \quad q_{z_{i} w_{k}}=q_{i} \text { for } k=1,2, \ldots, n .
$$

There is an action of $S_{m}$, on the corresponding cohomology space, whenever (2.2) is also satisfied; that is when $\alpha_{i j}, q_{i}$ are independent of $i, j$. Theorem 5.11 can now be applied to the situation where:

$$
\begin{aligned}
V & =\mathscr{C}^{m} /\left\langle\operatorname{Im}\left(\mathbf{D}_{1}^{T}\right), \ldots, \operatorname{Im}\left(\mathbf{D}_{m-1}^{T}\right)\right\rangle \\
& \left.\cong\langle(\underline{\alpha})| \underline{\alpha} \in \mathscr{S}_{w}^{m}, \alpha_{i} \neq z_{i+1} \text { for } i=1,2, \ldots, m-1\right\rangle \\
f(x) & =\operatorname{Im}\left(\mathbf{D}_{m}^{T}\right) .
\end{aligned}
$$

In the above $x \in M$ refers to $\left(\alpha_{i m}\right) \in \mathbf{C}^{m-1}$ with $q_{i}(i=1,2, \ldots, m)$ and $\alpha_{i j}(1 \leqq i<j<m)$ fixed. Here, the family $\mathbf{A}(x)$ used is given by the induced action of $\sigma_{i} \in B_{n}$ on the quotient $V$ of $\mathscr{C}^{m}$; that is, $A_{w_{i} w_{i+1}}^{(m) T^{-1}}$, where all the boundaries except those given in $\operatorname{Im}\left(D_{m}^{T}\right)$ have been divided out. The point $x_{0} \in M$ is given by $\alpha_{i j}=q_{i}^{-1} q_{j}^{-1}$.

Theorem 5.11 now gives a derived action of $B_{n}$ on the space $W / f\left(x_{0}\right) \subseteq H^{m}$, depending on the $m$ parameters $q_{1}, \ldots, q_{m}$. There is an action of $S_{m}$ on $\mathscr{C}^{m}$ (and thus also on $H^{m}$ ) only when $q_{i}=q \forall i$. In this case, the action:

defines a symmetriser:

$$
\begin{aligned}
S_{m} & \rightarrow \operatorname{End}\left(H^{m}\right) \\
(i i+1) & \mapsto q j_{i i+1}
\end{aligned}
$$

$$
\begin{aligned}
R: H^{m} & \rightarrow H^{m} \\
\mathbf{v} & \mapsto \frac{1}{m !} \sum_{\sigma \in S_{m}} \sigma(\mathbf{v}) .
\end{aligned}
$$

Thus $\operatorname{Im}(R)$ consists of that part of the cohomology $H^{m}$ invariant under the action of the symmetric group, $S_{m}$. The best way to construct the symmetric part of the reduced space $W / f\left(x_{0}\right)$, is as a subset of $\operatorname{Im}(R)$, transverse to $\operatorname{Im}\left(R \circ D f_{x_{0}}\right)$. Here, we are using the following maps: 


$$
\begin{aligned}
D f_{x_{0}}: T_{x_{0}} M \otimes f\left(x_{0}\right) & \rightarrow V / f\left(x_{0}\right) \cong H^{m} \\
R \circ D f_{x_{0}}: T_{x_{0}} M \otimes f\left(x_{0}\right) & \rightarrow H^{m} .
\end{aligned}
$$

Of course in order to apply Theorem 5.11, it is necessary to check all the conditions of that theorem. For generic $q, D f_{x_{0}}$ has maximal rank, as can be verified by using the recursion relations of Sect. 3, together with $q$ close to 1, but not equal to 1. To do this, it is necessary to identify $V$ with a suitable subspace of $\mathscr{C}^{m}$, and then to investigate the action of $\mathbf{D}_{m}^{T}$ on this space. Although $W$ needs to be introduced in Theorem 5.11, and this gives rise to an arbitrary element in the construction, the resultant derived braid group action obtained, is natural, since it can be expressed in terms of the action on the quotient $V /\left\langle f\left(x_{0}\right), D f_{x_{0}}\right\rangle$. We now make the following conjecture:

Conjecture 5.12. Consider the family of representations of $B_{n}$ on the cohomology with local coefficient system given by:

$$
q_{z_{i} z_{j}}=\alpha_{i j}, \quad q_{w_{k} z_{i}}=q
$$

( for $1 \leqq i, j \leqq m, 1 \leqq k \leqq n$ ), and parametrised by $\left\{\alpha_{i j}\right\}$. The symmetric part of the derived representation of this family at $\alpha_{i j}=q^{-2}$ factors through the Hecke algebra $H_{n}(q)$, giving the representation $\pi_{A_{m}}$.

In the case $m=2$, this conjecture has been proved in Sect. 4.2. In the general case, it is easily seen that $V^{m}$ is a subspace of the cohomology $H^{m}$ such that:

(a) $S_{m}$ preserves $V^{m}$; i.e. $V^{m}$ is contained in $\operatorname{Im}(R)$;

(b) $V^{m}$ is invariant under the action of $B_{n}$;

(c) for generic $q, \operatorname{Im}\left(D f_{x_{0}}\right)$ is transverse to $V^{m}$.

The last result is obtained by considering $q$ close to 1 , and using the basis for the space of chains, defined in Sect.3. Since all the matrices involved depend on $q$ in an analytic way (indeed, they are polynomials in $q$ and $q^{-1}$ ), it is possible to infer results for generic $q$ from those which hold for all $q \neq 1$, sufficiently close to 1 . A comparison of (a), (b), and (c) with the conditions of Theorem 5.11 shows that the derived action can be obtained on a space containing $V^{m}$; that is, $W$ can be chosen so that $W / f\left(x_{0}\right) \supseteqq V^{m}$, while $W$ satisfies the conditions (i)-(iii) of the theorem. By the remark above, the derived action is independent of $W$, and thus contains the action on $V^{m}$. Since $V^{m}$ is also preserved under the action of $S_{m}$, thus the action referred to in Conjecture 5.12 contains that of $B_{n}$ on $V^{m}$. By Theorem 5.1, we now obtain the following result.

Theorem 5.13. The symmetric part of the derived action of Conjecture 5.12 contains $\pi_{\Lambda_{m}}$ for all $m$ and generic $q$.

All that is necessary to obtain a proof of Conjecture 5.12 is to show that there is no other part to the totally symmetric piece of the derived representation. A dimension count would suffice for this; however, $\operatorname{dim} \operatorname{Im}\left(R \circ D f_{x_{0}}\right)$ is not simple to compute!

\subsection{Comparison with Tsuchiya-Kanie Theory}

In Sect. 2, natural actions of $B_{n}$ and $S_{m}$ on the homology space $H_{m}\left(Y_{\mathrm{w}, m}, \chi_{\mathrm{w}, m}(\mathbf{q})\right)$ were defined. As mentioned in Sect. 5 , there is naturally defined a dual action of $B_{n} \times S_{m}$ on the cohomology space $H^{m}\left(Y_{\mathrm{w}, m}, \chi_{\mathrm{w}, m}(\mathbf{q})\right)$. To obtain useful information from this point of view, it is necessary to express this cohomology space in terms of 
functions. Since $Y_{w, m}$ is the complement of a complex algebraic hypersurface in $\mathbf{C}^{m}$, it is a Stein manifold, and thus the cohomology can be calculated as the cohomology of the complex of holomorphic differentials. This result also holds when an abelian local coefficient system is introduced. Thus $H^{m}\left(Y_{\mathrm{w}, m}, \chi_{\mathrm{w}, m}(\mathbf{q})\right)$ (abbreviated to $\mathrm{H}^{m}$ ) can be computed in terms of the space:

$$
\mathcal{O}=\left\{f: Y_{\mathrm{w}, m} \rightarrow \mathbf{C} \mid f \text { is holomorphic and twists according to } \chi_{\mathbf{w}, m}(\mathbf{q})\right\}
$$

of holomorphic functions $f$ for which $f(\gamma(1))=\left(\chi_{\mathrm{w}, m}(\mathbf{q})\right)(\gamma) \cdot f(\gamma(0))$ for all $[\gamma] \in \pi_{1}\left(Y_{\mathrm{w}, m}\right)$. Hence:

$$
H^{m} \cong \mathcal{O} /\left\{\sum_{i=1}^{m} \frac{\partial f_{i}}{\partial z_{i}} \mid f_{i} \in \mathcal{O} \text { for } i=1,2, \ldots, m\right\} .
$$

By a theorem of Grothendieck (see $[\mathrm{ABG}, \mathrm{G}]$ ), this cohomology can be computed as the cohomology of algebraic differential forms. Let $g(\lambda, \mu)$ denote the function of $z_{1}, \ldots, z_{m}$ given by:

$$
\left(\prod_{i=1}^{m} \prod_{j=1}^{n}\left(z_{i}-w_{j}\right)^{-\mu_{i j}}\right)\left(\prod_{\substack{i, k=1 \\ i<k}}^{m}\left(z_{i}-z_{k}\right)^{-\lambda_{i k}}\right),
$$

where $\lambda=\left(\lambda_{i k}\right)$ and $\boldsymbol{\mu}=\left(\mu_{i j}\right)$. Any function $f \in \mathcal{O}$ can always be written as:

$$
f=g \cdot g^{0},
$$

where $g^{0}$ is the function $g(\lambda, \mu)$ with $\lambda_{i k}=a$ and $\mu_{i j}=b$ for all $i, j, k$; and $g$ is a holomorphic function $Y_{\mathrm{w}, m} \rightarrow \mathrm{C}$. Here $b, a$ are such that $e^{2 \pi i b}=q, e^{2 \pi i a}=\alpha$, so that $a=-2 b$ in the situation considered in Theorem 2.3. The space $\mathscr{R} \cong \mathcal{O}$, of algebraic differential forms, in the case in which the local coefficient system is non-trivial, is given by:

$$
\begin{aligned}
& \left\{g g^{0} \mid g \text { is a finite linear combination of } g(\lambda, \mu) \text {, where }\left\{\lambda_{i k}\right\}\right. \\
& \text { and } \left.\left\{\mu_{i j}\right\} \text { are all integers }\right\} \text {. }
\end{aligned}
$$

Any element $\underline{\alpha}$ of $\mathscr{S}_{w}^{m}$ defines:

$$
g_{\alpha}^{0}=\left(\prod_{i=1}^{m}\left(z_{i}-\alpha_{i}\right)^{-1}\right) \cdot g^{0} \in \mathscr{R} .
$$

Lemma 5.14. Suppose that $f$ is a function of the form $g(-\mathbf{a},-\mathbf{b}) \in \mathscr{R}$, where $\left\{a_{i k}\right\}$ and $\left\{b_{i j}\right\}$ differ from $a$ and $b$ by integers only. For $\underline{\alpha} \in \mathscr{S}_{w}^{m}$, define $f_{\alpha}$ by an equation similar to (5.9) in which $\mathrm{g}^{0}$ is replaced by $f$. Then, up to the equivalence of (5.10), $f_{z}\left(z_{x}-\lambda\right)^{-1}$ can be expressed as a combination of $f_{\beta}$ 's, where $w_{1}, \ldots, w_{n}$ are thought of as fixed and $\lambda \in\left\{z_{x+1}, \ldots, z_{m}, w_{1}, \ldots, w_{n}\right\}$. Furthermore, the coefficients of $f_{\beta}$ in $f_{a}\left(z_{x}-w_{j}\right)^{-1}$ can be expressed as constant linear combinations of $\left(w_{j}-w_{l}\right)^{-1}$ over l's not equal to $j$. Here $1 \leqq x \leqq m$.

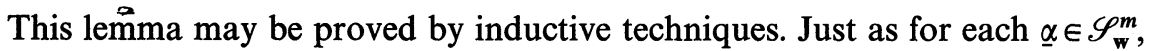
the chain $\gamma_{\alpha}$ was defined in Sect. 3.1, with $\left\{\gamma_{\alpha} \mid \underline{\alpha} \in \mathscr{S}_{w}^{m}\right\}$ providing a spanning set for $\mathscr{C}_{m}$, and ultimately for the homology $H_{m}$, similarly we have the following result for cohomology.

Lemma 5.15. The subset $\left\{g_{q}^{0} \mid \underline{\alpha} \in \mathscr{S}_{\mathbf{w}}^{m}\right\}$ of $\mathscr{R}$, when projected onto the cohomology $H^{m}$, provides a spanning set. 
Proof. By Grothendieck's Theorem, $H^{m}$ is the cohomology of the complex of differential forms based on the subset $\mathscr{R}$ of $\mathcal{O}$ defined in (5.8). It is thus given by a similar expression to (5.5), in which $\mathcal{O}$ has been replaced by $\mathscr{R}$. To verify the lemma it is thus sufficient to show that the function defined by (5.7), with $g=g(\lambda, \mu)$ (where $\left\{\lambda_{i k} \mid 1 \leqq i \leqq k \leqq n\right\},\left\{\mu_{i j} \mid 1 \leqq i \leqq m, 1 \leqq j \leqq n\right\}$ are subsets of the integers) is equivalent to a combination of $\gamma_{\alpha}$ 's with $\underline{\alpha} \in \mathscr{S}_{w}^{m}$, up to the equivalence " $\sim$ " in which:

$$
\frac{\partial f}{\partial z_{i}} \sim 0 \text { whenever } f \in \mathscr{R} .
$$

This result is obtained by repeated application of Lemma 5.14. At each stage, $\left\{a_{i k}\right\}$ and $\left\{b_{i j}\right\}$ differ from $a$ and $b$, by integer values, and the lemma is used to reduce $g g^{0}$ [where $g$ is defined in (5.7)] to a combination of similar functions,

$$
\left\{h g^{0} \cdot\left(\prod_{i=1}^{m}\left(z_{i}-\alpha_{i}\right)^{-1}\right) \mid \underline{\alpha \in \mathscr{S}_{\mathbf{w}}^{m}}\right\} \text {, }
$$

where $h$ is given by $g(\lambda, \mu)$ with each of $\lambda_{i k}, \mu_{i j}$ replaced by smaller integers (at least, integers no larger than $\lambda_{i k}, \mu_{i}^{j}$, respectively).

From the last two lemmas, it is apparent that the action of the braid group $B_{n}$ on cohomology can be computed from its action on $\left\{g_{\alpha}^{0} \mid \underline{\alpha} \in \mathscr{S}_{\mathbf{w}}^{m}\right\}$. Just as the homology can be embedded in $\left\langle\left\{\gamma_{\alpha} \mid \underline{\alpha} \in \mathscr{S}_{\mathrm{w}}^{m}\right\}\right\rangle$, similarly, in the cohomology $H^{m}$, $\left\{g_{q}^{0} \mid \underline{\alpha} \in \mathscr{S}_{w}^{m}\right\}$ is not a linearly independent set, although it does span $H^{m}$. Since $H_{m}$ and $H^{m}$ are dual, $\operatorname{dim} H_{m}=\operatorname{dim} H^{m}$ and thus the number of relations that exist between $\left\{\left[g_{q}^{0}\right]\right\}$ is identical to the dimension of the image of the boundary map $\delta: \mathscr{C}_{m} \rightarrow \mathscr{C}_{m-1}$ (whose kernel is $H_{m}$ ).

We now proceed to obtain a system of differential equations whose monodromy action is identical to that defined in Sect. 2. As w follows a path in $X_{n}$, the flat connection defined in Sect. 2 enables elements of the fibres over different points w to be identified, using parallel transport. In Sect. 2, it was seen how such an identification could lead to a representation of $B_{n}$ (and not just $P_{n}=\pi_{1}\left(X_{n}\right)$ ). This was accomplished using the natural identification of $Y_{w, m}$ and $Y_{w^{\prime}, m}$ which exists when $\mathbf{w}^{\prime}$ lies in the orbit of $\mathbf{w}$ under the action of $S_{n}$.

The functions $g_{\alpha}^{0}$ of (5.9) are defined for all $\alpha \in \mathscr{S}_{w}^{m}$, and give rise, over each $\mathbf{w} \in X_{n}$, to elements of the fibre $H^{m}\left(Y_{\mathbf{w}, m}, \chi_{\mathrm{w}, m}(\mathbf{q})\right)$ of $E_{m}(\mathbf{q})$. These functions vary holomorphically with w. By Lemma 5.15, any element of the cohomology can be represented as $[f]$, where:

$$
f=\sum_{\alpha \in \mathscr{S}_{m}^{m}} A_{\alpha} g_{\alpha}^{0}
$$

for some coefficients $A_{\alpha}$. In this relation, $\mathrm{w} \in X_{n}$ is fixed.

Theorem 5.16. For suitable constant matrices $\mathbf{C}_{j k}$, defined for each distinct pair of elements $j, k$ of $1,2, \ldots, n$, the system of differential equations:

$$
\frac{\partial \mathbf{A}}{\partial w_{j}}-\left(\sum_{k \neq j} \frac{\mathbf{C}_{j k}}{w_{j}-w_{k}}\right) \mathbf{A}=0
$$

for vector valued functions $\mathbf{A}$ on $X_{n}$, with $\left|\mathscr{S}_{\mathrm{w}}^{m}\right|$ components, has, as a solution, $\mathbf{A}=\left(A_{q}\right)$ only if the function $f$ defined by (5.11), is such that the associated elements $[f]$ of $H^{m}\left(Y_{\mathrm{w}, m}, \chi_{\mathrm{w}, m}(\mathbf{q})\right)$ define a flat section of the cohomology, with respect to the flat connection induced by that of Sect. 2 on homology. 
Proof. The second half of Lemma 5.14 states that there exists constants $C_{j k}^{(i)}(\beta, \underline{\alpha})$ such that:

$$
g_{\alpha}^{0}\left(z_{i}-w_{j}\right)^{-1}=\sum_{\beta} \sum_{\substack{k=1 \\ k \neq j}}^{n} C_{j k}^{(i)}(\beta, \alpha) \frac{g_{\beta}^{0}}{w_{j}-w_{k}},
$$

where the first sum is over all $\beta \in \mathscr{S}_{\mathbf{w}}^{m}$. From the definition of $g_{\alpha}^{0}$ in (5.9), $\frac{\partial g_{\alpha}^{0}}{\partial w_{j}}$ may be expressed as a linear combination of the expressions on the left-hand side above. The theorem follows immediately when this is combined with the fact that (5.11) defines a flat section if, and only if, $\frac{\partial f}{\partial w_{j}} \sim 0$ with respect to the equivalence relation “ " of (5.10).

Theorem 5.16 shows that for any solution $\mathbf{A}$ of (5.12), the corresponding element of cohomology defines a flat section of the vector bundle $E^{m}(\mathbf{q})$. The dimension of $W^{\prime} / \operatorname{Im}\left(\mathbf{D}^{\prime}\right)$ is less than the size of the vectors $\mathbf{A}$ in Theorem 5.16. Thus, the monodromy representation of $B_{n}$ given in the above theorem, is defined on a vector space, $X$, of dimension $\left|\mathscr{S}_{\mathrm{w}}^{\mathrm{m}}\right|$, and is much larger than the representation of Theorem 5.1.

Choose a fixed point $\mathbf{w}^{0} \in X_{n}$. Then (5.12) has $\left|\mathscr{S}_{\mathbf{w}}^{m}\right|$ linearly independent solutions. Let $Z$ denote the subspace of $X$ consisting of those $\mathbf{A}$ for which the corresponding element, $[f]$ of cohomology vanishes, where $f$ is given by (5.11). Then the solutions of (5.12) associated with $\mathbf{A}\left(\mathbf{w}^{0}\right) \in Z$ are all such that:

$$
\mathbf{A}(\mathbf{w}) \in Z
$$

for all $\mathrm{w} \in X_{n}$ in the orbit of $\mathrm{w}^{0}$, under the action of $S_{n}$ on $X_{n}$. The monodromy action obtained from (5.12) thus preserves $Z$, and the induced action of $B_{n}$ on $X / Z$ is identical to the monodromy representation obtained on cohomology.

Let $Y$ denote the subspace of $X$ consisting of those $\mathbf{A} \in X$ for which:

$$
\left(\int_{\gamma_{\underline{\alpha}}} f\right)=\mathbf{y}+\mathbf{z}
$$

where $f$ is the associated element of $\mathscr{C}^{m}$ defined by (5.11); y lies in the image of $\operatorname{Im}\left(\mathbf{D}^{\prime}\right) \cong \mathscr{C}^{m}$ under the map $g \rightarrow\left(\int_{\gamma_{\underline{\alpha}}} g\right)$; and $\mathbf{z}$ is a vector whose components associated with $\underline{\alpha} \in \mathscr{S}_{\mathbf{w}}^{m} \backslash \mathscr{T}_{\mathrm{w}}^{m}$ vanish. In this definition, $\mathbf{y}$ and $\mathbf{z}$ are vectors with $\left|\mathscr{S}_{\mathbf{w}}^{m}\right|$ components. This rather elaborate definition is analogous to that of $W^{\prime} \cong \mathscr{C}^{m}$ in Sect. 5.1. Indeed, $[f] \in W^{\prime} / \operatorname{Im}\left(\mathbf{D}^{\prime}\right)$ is equivalent to $A \in Y$.

There is a natural action of $S_{m}$ on $X$ induced by the action on homology in Sect. 2, and this preserves the subspaces $Z$ and $Y$. The space $(Y)^{S_{m}} /(Z)^{S_{m}} \cong(Y / Z)^{S_{m}}$ has the same dimension as that on which the monodromy action of Theorem 5.1 acts, namely, $W^{\prime} / \operatorname{Im}\left(\mathbf{D}^{\prime}\right)$. From the above definitions of $Z$ and $Y$, it follows that the monodromy action of Theorem 5.1 is obtained from that given by Theorem 5.16, by inducing the action of $B_{n}$ on $X$, onto $(Y / Z)^{S_{m}}$ at $\mathbf{w}=\mathbf{w}_{0}$. Since (5.12) ensures that, for any solution, the associated function $f$ has $\int_{\gamma_{\underline{\alpha}}} f$ constant in $\mathbf{w}$, for all $\underline{\alpha} \in \mathscr{S}_{\mathbf{w}}^{m}$ (see Theorem 5.16), it is clear that the induced monodromy action preserves both $Z$ and $Y$.

Lemma 5.17. The subspaces $Z$ and $Y$ of $X$ are independent of $w$. 
Note also that the action of $S_{m}$ on $X$ is independent of $\mathbf{w}$; in fact, the matrices giving the action of $\sigma \in S_{m}$ on $X$, in terms of the standard basis, have all their elements $1,-1$, or 0 . Thus $(Y / Z)^{S_{m}}$ is a constant quotient of a subspace of $X$. Since this space is invariant under the flow defined by (5.12), then the induced monodromy action of $B_{n}$ on $(Y / Z)^{S_{m}}$ is given by the total monodromy action of a similar system of differential equations to (5.12). In particular $\mathbf{C}_{j k}$ gives a well defined action on $(Y / Z)^{S_{m}}$. We thus deduce:

Corollary 5.18. The representation of Theorem 5.1 obtained by restricting the monodromy action on cohomology to $\left(W^{\prime} / \operatorname{Im}\left(\mathbf{D}^{\prime}\right)\right)^{S_{m}} \subseteq H^{m}$, can also be obtained as the monodromy representation associated with the system of equations:

$$
\frac{\partial \mathbf{A}}{\partial w_{j}}-\left(\sum_{j \neq k} \frac{\mathbf{c}_{j k}}{w_{j}-w_{k}}\right) \mathbf{A}=0,
$$

where $\mathbf{A}$ is a vector-valued function on $X_{n}$, with constant matrices $\mathbf{c}_{j k}$.

This may be compared with the construction of the representation $\pi_{\Lambda_{m}}$ by Tsuchiya and Kanie; see [TK]. They obtained this representation from the monodromy representation of $B_{n}$ on a space of $n$-point functions. This is found to be given by the system of linear differential equations:

$$
\left(\kappa \frac{\partial}{\partial z_{i}}-\sum_{\substack{k=1 \\ k \neq i}}^{n} \frac{\boldsymbol{\Omega}_{i k}}{z_{i}-z_{k}}\right) \mathbf{f}\left(z_{1}, \ldots, z_{n}\right)=0,
$$

where $\mathbf{f}$ is a holomorphic vector valued function $X_{n} \rightarrow V$ and $\boldsymbol{\Omega}_{i k}$ denote polarisations of a Casimir operator. Let $V_{j}$ denote a space on which the spin $j$ representation of $\mathfrak{s l}_{2}$ acts. Then we let $V$ denote the $\mathfrak{s l}_{2}$-invariant part of $V_{1 / 2}^{\otimes n} \otimes \breve{V}_{t}$, while $t=n / 2-m$. Let $H, E, F$ denote the standard generators of $\mathfrak{s I}_{2}$, and:

$$
\mathbf{\Omega}_{i k}=\frac{1}{2} \pi_{i}(H) \pi_{k}(H)+\pi_{i}(E) \pi_{k}(F)+\pi_{i}(F) \pi_{k}(E),
$$

where $\pi_{i}$ denotes the action of $\mathfrak{s l}_{2}$ on the $i^{\text {th }}$ factor in $V$. The representation obtained from (5.14) is found to factor through $H_{n}(q)$, where $q=\exp (2 \pi i / \kappa)$. It may be verified that the dimension of $V$ is:

$$
\left(\begin{array}{c}
n \\
n / 2-t
\end{array}\right)-\left(\begin{array}{c}
n \\
n / 2-t-1
\end{array}\right)
$$

which is therefore identical with that of $V^{m}$. There are thus two similar systems of differential equations, both giving rise to the same representation of $B_{n}$, namely, that representation, $\pi_{A_{m}}$, which factors through $H_{n}(q)$ and is associated with the Young diagram $\Lambda_{m}$. Such a system of differential equations is equivalent to the condition of flatness of a section of a vector bundle over $X_{n}$, on which there is a flat connection specified by the 1-form:

$$
\nabla\left(e_{\lambda}\right)=-\sum_{\mu=1}^{N} \omega_{\mu \lambda} \otimes e_{\mu} .
$$

Here $N=\operatorname{dim} V$ and $\left(e_{\lambda}\right)$ is a basis for $V ; \boldsymbol{\omega}=\left(\omega_{\lambda \mu}\right)$ is the matrix valued 1-form defined by:

$$
\boldsymbol{\omega}=\sum_{i<j} \boldsymbol{\Omega}_{i j} d \ln \left(z_{i}-z_{j}\right),
$$

the sum being over all $i, j \in\{1,2, \ldots, n\}$ with $i<j$, while $\boldsymbol{\Omega}_{i j}$ are constant matrices. Kohno [Ko] has shown the following lemma. 
Lemma 5.19. The monodromy representation $\theta_{\mathbf{\Omega}}$ of $B_{n}$ associated with the connection given by (5.15) and (5.16), with the $\boldsymbol{\Omega}_{i j}$ matrices small, depends on $\left\{\boldsymbol{\Omega}_{i j}\right\}$ injectively. That is, if $\theta_{\mathbf{\Omega}}$ and $\theta_{\mathbf{\Omega}^{\prime}}$ are identical then $\boldsymbol{\Omega}=\mathbf{\Omega}^{\prime}$, so long as $\boldsymbol{\Omega}_{i j}, \boldsymbol{\Omega}_{i j}^{\prime}$ are small, in the sense that the maximum entries in $\boldsymbol{\Omega}_{i j}$ and $\boldsymbol{\Omega}_{i j}^{\prime}$ are all sufficiently close to zero.

Theorem 5.20. There exists an isomorphism $\alpha: V_{0} \rightarrow\left(W^{\prime}\right)^{S_{m}}$ such that:

$$
\kappa^{-1} \alpha \circ\left(\boldsymbol{\Omega}_{i j}-\frac{1}{2} \mathbf{I}\right)=\mathbf{c}_{i j} \circ \alpha,
$$

where $\mathbf{c}_{i j}$ are the matrices of Corollary 5.18, where $m=n / 2-t, q=e^{2 \pi i /(l+2)}$.

Proof. The differential equations (5.14) and (5.12) (or at least, the reduced system (5.13), given by Corollary 5.18) both give rise to representations of $B_{n}$ which factor through $H_{n}(q)$. They both correspond to two-row Young diagrams, with $n$ squares in total, namely $\Lambda_{n / 2-t}$ and $\Lambda_{m}$, respectively. Thus when $m=n / 2-t$, the two braid group representations are isomorphic.

When $l \rightarrow \infty, \kappa^{-1} \boldsymbol{\Omega}_{i j} \rightarrow 0$ in the Tsuchiya-Kanie side of the story. On the other hand, this is associated with $q \rightarrow 1$ and in this limit the local coefficient system is trivial. Thus in the proof of Theorem 5.16,

$$
\frac{\partial g_{a}^{0}}{\partial w_{j}} \sim \sum_{i=1}^{m} \frac{\delta_{\alpha_{i} w_{j}} g_{\alpha}^{0}}{z_{i}-w_{j}} .
$$

The only terms that occur here are thus of the form:

$$
\left(z_{1}-\alpha_{1}\right)^{-1} \ldots\left(z_{i-1}-\alpha_{i-1}\right)^{-1}\left(z_{i}-\alpha_{i}\right)^{-2}\left(z_{i+1}-\alpha_{i+1}\right)^{-1} \ldots\left(z_{m}-\alpha_{m}\right)^{-1} \text {, }
$$

and such terms are equivalent to 0 under “ " of (5.10). Thus $\frac{\partial g_{\alpha}^{0}}{\partial w_{j}} \sim 0$ for all $\underline{\alpha \in \mathscr{S}_{w}^{m}}$ and $j \in\{1,2, \ldots, n\}$. Hence $\mathbf{C}_{j k}=\mathbf{0}$ in (5.12), and so $\mathbf{c}_{j k}=0$ in Corollary 5.18. Since $\mathbf{c}_{j k}$ are rational functions of $q$, thus, for sufficiently small $q, \kappa^{-1} \boldsymbol{\Omega}_{j k}$ and $\mathbf{c}_{j k}$ will both be small and give rise to the same monodromy representations of $B_{n}$. By Lemma 5.19, the matrices $\kappa^{-1} \boldsymbol{\Omega}_{j k}$ and $\mathbf{c}_{j k}$ must therefore be equivalent.

We conclude this section with a dictionary of the correspondence between Tsuchiya-Kanie theory and the homology theory defined in Sect. 2, as derived in Theorem 5.20.

\begin{tabular}{ll}
\hline Tsuchiya-Kanie theory & Homology theory \\
\hline$t$ & $n / 2-m$ \\
$V_{0}$ & $(Y / Z)^{S_{m}} \cong\left(W^{\prime} / \operatorname{Im}\left(\mathbf{D}^{\prime}\right)\right)^{S_{m}} \cong V^{m}$ \\
$\mathbf{\Omega}_{i j} / \kappa$ & $\mathbf{c}_{i j}$ \\
$\kappa=l+2$ & $2 \pi i /(\ln q)$ \\
Construction of $[\mathrm{TK}]$ & Theorem 5.1 \\
Equation (5.14) & Theorem 5.16 \\
Kohno connection & Natural connection on cohomology \\
\hline
\end{tabular}




\section{Further Remarks}

Throughout the last few sections, it has been assumed that $q$ is not a root of unity, or equivalently, that $l$ is not an integer. When $l$ is integral, Tsuchiya and Kanie showed how introducing an extra system of algebraic relations, in addition to (5.17), gives rise to irreducible Hecke algebra representations, once again. This suggests that a similar such construction should exist in terms of the homology picture. In Sect. 4.3, it was observed that in the special case $q=1$, the representation of $S_{n}$ constructed on $\left(W^{\prime}\right)^{S_{m}}$ is larger than the irreducible representation $\pi_{A_{m}}$. At other roots of unity a similar degeneracy can occur, when the homology is computed; that is, $\mathbf{D}^{\prime}$ may not have maximal rank. This is to be compared with the situation discussed above, at roots of unity, in the theory of Tsuchiya and Kanie.

In both approaches to the construction of $\pi_{\Lambda_{m}}$, the Hecke algebra representation required appears as a sub-representation of a much larger braid group representation. In Tsuchiya and Kanie's approach, the natural action of $B_{n}$ on $V_{1 / 2}^{\otimes n}$ gives the larger space. In the homology approach, the larger representation appears on the symmetric part of the cohomology (with the dual representation on the symmetric part of the homology). The dimension of the reduced representation is:

$$
\left(\begin{array}{l}
n \\
m
\end{array}\right)-\left(\begin{array}{c}
n \\
m-1
\end{array}\right)
$$

and those of the larger representations are $2^{n}$ and $\frac{1}{m !}(n+m-2) \ldots n(n-1)$, respectively.

In Sects. 4.2, 5.3 it was shown how, in certain special cases, a reduced representation of $B_{n}$ could be constructed from the family of representations with parameter $\alpha$, by looking near to $\alpha=q^{-2}$. The representation of $B_{n}$ associated with generic values of $\alpha$ and $q$, is irreducible. When $\alpha=q^{-2}$, it is reducible, one part being the required Hecke algebra representation. There is a similarity here with the behaviour of the Hecke algebra representations as $q$ varies. Generically $\pi_{\Lambda_{m}}$ is irreducible. However, when $q$ is a root of unity, this representation may be reducible, and when it is, the representation constructed by Tsuchiya and Kanie is a large irreducible piece of it. This leads one to speculate on how such an irreducible piece may be isolated. Tsuchiya and Kanie do this by adding an extra system of equations. If the analogy is valid, one would expect that it may be possible to select the sub-representation out by using a limiting lemma, along the lines of Theorem 5.11.

As far as the Jones polynomial of links is concerned, $V_{L}$ can be expressed as a combination of the characters $\chi_{\Lambda_{m}}$, evaluated on a braid whose closure is the link concerned; see $[\mathrm{J}]$. When $q$ is not a root of unity, these characters correspond to irreducible representations; but when $q$ is a root of unity, it is still these characters, and not their decompositions into irreducible parts, which play the central role. Thus although it should be possible to construct, in a natural topological manner, the irreducible parts of $\chi_{A_{m}}$, for $q$ a root of unity, this would have no significance as far as a topological interpretation of $V_{L}$ is concerned. However, the generalisations of the Jones polynomial given in Witten's theory [Wi] (see also [S1, S2 and S3]) are in terms of the (smaller) irreducible representations. In the case of the Jones polynomial, the extra parts of the representations cancel out, and so, we can 
equally well use the larger representation, $\pi_{\Lambda_{m}}$, as its irreducible subrepresentation. A topological construction of the Jones polynomial $V_{L}$, using a functional description along the lines of Turaev $[\mathrm{Tu}]$, but replacing $R$-matrices and quantum groups by abelian coefficient systems, will be presented in a another paper [L2].

The procedure outlined in this paper for constructing $\pi_{A_{m}}$, should be thought of as an abelianisation of the methods of [TK], in which the Kohno connection has been replaced by an abelian non-trivial local coefficient system. This should be compared with the abelianisation procedure employed to investigate Witten's description (see [Wi]) of the Jones polynomial using topological quantum field theory, in the work of Hitchin [H1, H2].

Acknowledgements. I would like to acknowledge the encouragement of my supervisor Sir Michael Atiyah while working on my D. Phil. thesis at Oxford, on which this paper is based. I would also like to thank my thesis examiners, Graeme Segal and John Jones, for useful comments and suggestions.

\section{References}

[A] Atiyah, M.F.: The geometry and physics of knots. Lezioni Lincee. Cambridge: University Press (1990)

[ABG] Atiyah, M.F., Bott, R., Gårding, L.: Lacunas for hyperbolic differential operators with constant coefficients. Acta Mathematica 124, 109-189 (1970)

[A11] Alexander, J.: Topological invariants of knots and links. Trans. AMS 30, 275-306 (1928)

[A12] Alexander, J.W.:A lemma on a system of knotted curves. Proc. Nat. Acad. Sci. USA 9, 93-95 (1923)

[FYHLMO] Freyd, P., Yetter, D., Hoste, J., Lickorish, W., Millet, K., Ocneanu, A.: A new polynomial invariant of knots and links. Bull. AMS 12, 239-246 (1985)

[G] Grothendieck, A.: On the de Rham cohomology of algebraic varieties. Publ. IHES 29, 351-359 (1966)

[H1] Hitchin, N.: The self-duality equations on a Riemann surface. Proc. Lond. Math. Soc. 55, 59-126 (1987)

[H2] Hitchin, N.: Reduction to the Abelian case. Oxford Seminar on Jones-Witten Theory 75-92 (1988)

[J] Jones, V.F.R.: Hecke algebra representations of braid groups and link polynomials. Ann. Math. 126, 335-388 (1987)

[Ko] Kohno, T.: Hecke algebra representations of braid groups and classical YangBaxter equations. Adv. Stud. Pure Maths. 16, 255-269 (1988)

[L1] Lawrence, R.J.: Homology representations of braid groups. D.Phil. Thesis, Oxford (June 1989)

[L2] Lawrence, R.J.: A functional approach to the one-variable Jones polynomial. Harvard University preprint (1990)

[M] Markov, A.: Über die freie Äquivalenz geschlossener Zöpfe. Recueil Math. Moscow 1, 73-78 (1935)

[R] Rolfsen, D.: Knots and links. Publish or Perish Press 1976

[S1] Segal, G.B.: Two-dimensional conformal field theories and modular functors. Proc. IXth Int. Congr. on Mathematical Physics 22-37 (1989)

[S2] Segal, G.B.: The Abelian Theory. Oxford Seminar on Jones-Witten Theory 17-35 (1988)

[S3] Segal, G.B.: Fusion rules and the Verlinde algebra. Oxford Seminar on JonesWitten Theory 51-74 (1988) 
[TK] Tsuchiya, A., Kanie, Y.: Vertex operators in conformal field theory on $\mathbf{P}^{1}$ and monodromy representations of braid groups. Adv. Stud. Pure Math. 16, 297-372 (1988), Erratum ibid 19, 675-682 (1990)

[Tu] Turaev, V.G.: The Yang-Baxter equation and invariants of links. Invent. Math. 92, 527-553 (1988)

[We] Wenzl, H.: Hecke algebra representations of type $A_{n}$ and subfactors. Invent. Math. 92, 349-383 (1988)

[Wi] Witten, E.: Some geometrical applications of quantum field theory. IXth Int. Congr. on Mathematical Physics 77-116 (1989)

Communicated by N. Yu. Reshetikhin

Note added in proof: Subsequent to [L1], Schechtman and Varchenko [SV] carried out an analysis of equations of Knizhnik-Zamolodchikov type for general Lie algebras g. In particular, (5.14) with $\boldsymbol{\Omega}_{i k}$ replaced by a polarisation of the Casimir operator for $\mathfrak{g}$ (instead of $\mathfrak{s l}_{2}$ ), was shown to have solutions which could be expressed by an integral representation, using suitably twisted functions generalising those of (5.9) in Sect. 5.4. The significance of the special twisting $\chi(q)$ given by $\alpha=q^{-2}$ in Theorem 5.1 (see (2.4)) is then seen to fit into a much more general picture involving the Cartan matrix of the Lie algebra concerned. The algebraical relations of Tsuchiya and Kanie referred to in Sect. 6, when translated into the language of twisted functions are given in [FSV] for the case of general Lie algebras.

\section{References}

[FSV] Feigen, B.L., Schechtman, V.V., Varchenko, A.N.: On algebraic equations satisfied by correlators in Wess-Zumino-Witten models. Preprint

[SV] Schechtman, V.V., Varchenko, A.N.: Integral representations of $n$-point conformal correlators in the WZW model. Preprint MPI/89-51 
